\title{
Topological properties of Hilbert schemes of almost-complex four-manifolds II
}

\author{
JULIEN GRIVAUX
}

\begin{abstract}
In this article, we study the rational cohomology rings of Voisin's Hilbert schemes $X^{[n]}$ associated with a symplectic compact four-manifold $X$. We prove that these rings can be universally constructed from $H^{*}(X, \mathbb{Q})$ and $c_{1}(X)$, and that Ruan's crepant resolution conjecture holds if $c_{1}(X)$ is a torsion class. Next, we prove that for any almost-complex compact four-manifold $X$, the complex cobordism class of $X^{[n]}$ depends only on the complex cobordism class of $X$.
\end{abstract}

32Q60; 14C05, 14J35

\section{Introduction}

The Hilbert schemes of points $X^{[n]}$ of a smooth projective complex surface $X$ are moduli spaces for finite subschemes of length $n$ on $X$. By a result of Fogarty [16], the varieties $X^{[n]}$ are smooth crepant resolutions of the $n$-fold symmetric powers $X^{(n)}$ of $X$, so that they present a strong geometric interest. Hilbert schemes of points have been intensively studied in the past twenty years, and this has led to important developments in algebraic and differential geometry as well as in theoretical physics (see Iarrobino [24] and Göttsche [19] for an overview).

Among these various studies, we will recall here what concerns the cohomology rings $H^{*}\left(X^{[n]}, \mathbb{Q}\right)$ of Hilbert schemes.

The first step towards the understanding of the vector spaces $H^{*}\left(X^{[n]}, \mathbb{Q}\right)$ was achieved by Göttsche [17] with the computation of the generating series for the Betti numbers $b_{i}\left(X^{[n]}\right)$ in terms of the Betti numbers of the surface $X$. Then Wafa and Witten pointed out that the infinite-dimensional vector space $\mathbb{H}=\bigoplus_{n \geq 0} H^{*}\left(X^{[n]}, \mathbb{Q}\right)$ was (by Göttsche's formula) an abstract highest-weight representation of the Heisenberg superalgebra modeled on $H^{*}(X, \mathbb{Q})$. Such a construction was geometrically realized independently by Nakajima [34] and by Grojnowski [23], using correspondences given by incidence varieties. The additive structure of the cohomology rings of Hilbert schemes of points was thus given a precise geometric description. 
Then Lehn obtained in [25] a decisive result in the study of the multiplicative structure. He computed explicitly, when $E$ is an algebraic vector bundle on $X$ and the $E^{[n]}$ are the associated tautological bundles on the $X^{[n]}$, the cup product by $\operatorname{ch}\left(E^{[n]}\right)$ on $H^{*}\left(X^{[n]}, \mathbb{Q}\right)$, and described in this way the subring of $\mathbb{H}$ generated by the Chern classes of all the tautological bundles. This study was completed by Li, Qin and Wang [29], who constructed virtual tautological Chern characters $G(\alpha, n)$ attached to each cohomology class $\alpha$ on $X$, and computed the cup product by each $G(\alpha, n)$ on $\mathbb{H}$. The classes $G(\alpha, n)$ extend the Chern characters of the tautological bundles in the following way: if $\alpha$ is the Chern character of an algebraic vector bundle $E$, then $G(\alpha, n)$ is the Chern character of $E^{[n]}$. Besides, it is shown in [29] that the components $\left(G_{i}(\alpha, n)\right)_{0 \leq i<n}$ of the classes $G(\alpha, n)$ generate the ring $H^{*}\left(X^{[n]}, \mathbb{Q}\right)$ when $\alpha$ runs through a basis of $H^{*}(X, \mathbb{Q})$. Using these generators, the authors obtained in [30] a universal description of $H^{*}\left(X^{[n]}, \mathbb{Q}\right)$ from the ring $H^{*}(X, \mathbb{Q})$ and the first Chern class of $X$ in $H^{2}(X, \mathbb{Q})$.

This study led to interesting consequences in the particular case where $X$ is a $K 3-$ surface. Indeed, at the same time, Chen and Ruan [9] developed the theory of orbifold cohomology and hinted at the existence of strong relations between the orbifold cohomology ring of an orbifold and the cohomology ring of a crepant resolution: this is known as the cohomological crepant resolution conjecture (see Ruan [38]). If $X$ is a $K 3$-surface, the Hilbert schemes $X^{[n]}$ are hyperkähler by a result of Beauville [4] and in this case the cohomological crepant resolution conjecture predicts that for every positive integer $n$, the ring $H^{*}\left(X^{[n]}, \mathbb{C}\right)$ and the orbifold cohomology ring $H_{\text {orb }}^{*}\left(X^{(n)}, \mathbb{C}\right)$ of the $n$-fold symmetric product of $X$ are isomorphic (see Adem, Leida and Ruan [1, Conjecture 4.24]). The above recalled description of the cohomology ring of Hilbert schemes made it possible to prove this prediction (see [27]) by putting together results of Lehn and Sorger [27] for the Hilbert schemes part with the computations performed independently by Fantechi and Göttsche [15] and Uribe [42] for the orbifold part.

If we leave the algebraic setting and consider abstract compact complex surfaces instead of projective ones, Hilbert schemes of points still exist (they are usually called Douady schemes). If $X$ is a compact Kähler surface, it can be deformed to a projective surface, so that the general description given for the cohomology rings of $X^{[n]}$ when $X$ is projective remains valid. In the general case of possibly non-Kähler compact complex surfaces, Göttsche's formula has been proved by de Cataldo and Migliorini [7], but the study of the multiplicative structure cannot be performed as in the projective case. Indeed, Lehn [25] uses in an essential way the fact that the cohomological cycle classes of smooth algebraic curves on a smooth projective surface $X$ span $H^{1,1}(X)$. This property fails for abstract complex compact surfaces. 
In a still more general context, Voisin defined in [43] Hilbert schemes $X^{[n]}$ associated with any almost-complex four-manifold $(X, J)$ : for every positive integer $n, X^{[n]}$ is a stably almost-complex differentiable manifold of real dimension $4 n$. These almostcomplex Hilbert schemes are symplectic if $X$ is symplectic (see Voisin [44]), and are still crepant resolutions of the $n$-fold symmetric products of $X$. This construction sheds a new light on the results we have mentioned about the cohomology rings of Hilbert schemes: indeed, Voisin's results imply that, for any projective surface $X$ and any positive integer $n$, the underlying differentiable manifold of $X^{[n]}$ depends only on the underlying differentiable manifold of $X$ and on the deformation class of the complex structure of $X$ in the space of almost-complex structures. This explains why the ring $H^{*}(X, \mathbb{Q})$ depends only on almost-complex invariants of $X$. On the other hand, it is worth noticing that orbifold cohomology is naturally defined for almost-complex orbifolds, which includes $n$-fold symmetric products of any almost-complex manifold.

Our paper is the second part of a program, the aim of which is the study of Voisin's almost-complex Hilbert schemes. The first part [21] has been devoted to the additive structure of their cohomology rings: Göttsche's formula has been proved and Nakajima operators have been constructed. The first main concern here is the study of the ring structure of the almost-complex Hilbert schemes. We prove the analog of Li, Qin and Wang's result quoted above under a symplectic hypothesis:

Theorem 1.1 If $(X, J)$ is a symplectic four-manifold, the rings $H^{*}\left(X^{[n]}, \mathbb{Q}\right)$ can be constructed by universal formulae from the ring $H^{*}(X, \mathbb{Q})$ and the first Chern class of $X$ in $H^{2}(X, \mathbb{Q})$.

This theorem is proved in Section 5.3. Let us have a glimpse at the strategy of the proof. The techniques developed in [21] allow us to adapt in the almost-complex case the quasitotality of the proof of Lehn's main formula [25, Theorem 3.10], except for the very argument which has already been pointed out for non algebraic complex surfaces: if $(X, J)$ is an almost-complex compact four-manifold, the homology classes of smooth $J$-holomorphic curves in $X$ do not span $H_{2}(X, \mathbb{Q})$ in general. To overcome this difficulty, we use the symplectic assumption: if $X$ is symplectic and if $J$ is an adapted almost-complex structure, Donaldson's theorem on symplectic divisors [11] makes it possible to span $H_{2}(X, \mathbb{Q})$ by pseudoholomorphic curves for small perturbations of the almost-complex structure $J$.

The second important problem in the proof of Theorem 1.1, which occurs only if the first Betti number of $X$ is nonzero, is to construct virtual tautological characters for almost-complex Hilbert schemes. Indeed, in the classical situation, if $X$ is a projective surface, and if $Y_{n}$ is the incidence locus in $X^{[n]} \times X$, the virtual tautological 
Chern character $G(\alpha, n)$ is equal to $\operatorname{pr}_{1 *}\left[\operatorname{ch}\left(\mathcal{O}_{Y_{n}}\right) \cdot \operatorname{pr}_{2}^{*}(\alpha \cdot \operatorname{td}(X))\right]$. The problem in the almost-complex setting lies in the term $\operatorname{ch}\left(\mathcal{O}_{Y_{n}}\right)$. Unlike in the case of vector bundles, there is no tractable analog of coherent analytic sheaves on almost-complex Hilbert schemes: the differentiable structure of $X^{[n]}$ is pretty hard to deal with; the topological structure of $X^{[n]}$ (as a $C^{0}$-manifold) would do better, but sheaves of continuous functions are generally ill-behaved. To explain the means used to cope with this problem, we have to recall the basics of the construction of $X^{[n]}$ when $X$ is an almost-complex four-manifold: it relies on the choice of a relative integrable complex structure $J_{n}^{\text {rel }}$, which is essentially a smooth family $J_{n, x}^{\text {rel }}$ of integrable complex structures parameterized by $X^{(n)}$ such that for each $\underline{x}$ in $X^{(n)}, J_{n, x}^{\text {rel }}$ is an integrable complex structure in a neighbourhood $W_{\underline{x}}$ of the points of $\underline{x}$. If $W_{\mathrm{rel}}^{[n]}$ is the disjoint union of the Hilbert-Douady schemes $\underline{W}_{\underline{x}}^{[n]}$, where each $W_{\underline{x}}$ is endowed with the integrable structure $J_{n, \underline{x}}^{\mathrm{rel}}$, then $X^{[n]}$ is a subset of $W_{\mathrm{rel}}^{[n]}$. Our main idea is to replace $X^{[n]}$ by $W_{\text {rel }}^{[n]}$, the latter having a much better structure: it is a differentiable orbifold fibred in smooth analytic sets over $X^{(n)}$. We develop in a systematic way a theory for these spaces, which we call relative analytic spaces, and for a particular class of sheaves on them, the relatively coherent sheaves. These sheaves are locally an extension of classical coherent analytical sheaves by $\mathcal{C}^{\infty}$ parameters. If $T$ is a differentiable orbifold chosen as parameter space and if $\mathcal{F}$ is a coherent analytic sheaf on an analytic set $Z$, then it is possible to define a sheaf $\mathcal{C}^{\infty}(T, \mathcal{F})$ of smooth sections of $\mathcal{F}$ with parameters in $T$ as follows: if $\mathcal{F}$ is the sheaf $\mathcal{O}_{Z}$, then $\mathcal{C}^{\infty}\left(T, \mathcal{O}_{Z}\right)$ is the subsheaf of $\operatorname{pr}_{1 *} \mathcal{C}_{Z \times T}^{\infty}$ consisting of smooth functions holomorphic in the first variables, and for an arbitrary $\mathcal{F}$, the sheaf $\mathcal{C}^{\infty}(T, \mathcal{F})$ is equal to $\mathcal{F} \otimes_{\mathcal{O}_{Z}} \mathcal{C}^{\infty}\left(T, \mathcal{O}_{Z}\right)$. Besides, this construction can be sheafified in the space of parameters: if $V$ is a differentiable orbifold, there exists a sheaf $\tilde{\mathcal{F}}$ on $Z \times V$ such that for all open subsets $U$ and $T$ of $Z$ and $V$ respectively, $\Gamma(U \times T, \widetilde{\mathcal{F}})$ is equal to $\Gamma\left(U, \mathcal{C}^{\infty}(T, \mathcal{F})\right)$. In our construction, $Z \times V$ is a local model for a relative analytic space and $\tilde{\mathcal{F}}$ is a local model for a relatively coherent sheaf on $Z \times V$. A relative analytic space is obtained by gluing together a family of such local models and so is a relatively coherent sheaf.

The formalism of relative analytic spaces and relatively coherent sheaves allows us to use tools of algebraic and analytic geometry in the almost-complex setting. It does not only solve our present problem, but will be essential in the last section of the paper (Section 6). We give an independent exposition of this formalism, as it may be useful in other situations.

As soon as Lehn's formula is obtained and virtual Chern characters are constructed, Theorem 1.1 follows from formal combinatorial arguments. We also prove (in Section 5.3) that Ruan's cohomological crepant resolution conjecture is valid for Hilbert schemes of symplectic compact four-manifolds with torsion first Chern class: 
Theorem 1.2 Let $(X, \omega)$ be a symplectic compact four-manifold with vanishing first Chern class in $H^{2}(X, \mathbb{Q})$. Then, for every positive integer $n$, the rings $H^{*}\left(X^{[n]}, \mathbb{C}\right)$ and $H_{\text {orb }}^{*}\left(X^{(n)}, \mathbb{C}\right)$ are isomorphic.

Our second object in this paper is the description of the complex cobordism classes of almost-complex Hilbert schemes. We obtain:

Theorem 1.3 Let $(X, J)$ be an almost-complex compact four-manifold. For any positive integer $n$, the complex cobordism class of $X^{[n]}$ given by its stable almostcomplex structure depends only on the complex cobordism class of $X$.

This theorem is proved in Section 6. Our interest in this problem goes back to Voisin's original motivation for constructing Hilbert schemes in the almost-complex setting: it is the computation of the cobordism classes of Hilbert schemes of points for projective surfaces, achieved by Ellingsrud, Göttsche and Lehn in [14]. The authors proved that these classes can be universally computed from the cobordism classes of the surface $X$ itself. Their result shows that Hilbert schemes of points for projective surfaces can be interpreted as modifications at the level of complex cobordism. Voisin's idea was that this modification of the complex cobordism could be lifted at the level of almost-complex manifolds. Although she actually constructed Hilbert schemes for almost-complex four-manifolds, it is not at all clear that they actually lift the classical Hilbert schemes at the cobordism level. Our theorem means that it is indeed the case. The proof relies heavily on the use of relatively coherent sheaves to adapt the argument of [14] in the almost-complex setting.

Let us now describe the organization of the paper.

Two distinct aims are pursued in Section 2. The first one is to define relative analytic spaces, which occur in particular in Voisin's construction of almost-complex Hilbert schemes. The second one is to recall this construction as well as related results. This section, which is mainly expository, will be used throughout the paper. In Section 2.1, we recall classical results about Hilbert schemes of points. In Section 2.2, we introduce relative and differentiable analytic spaces. This section consists mostly of definitions. The link between relative analytic spaces and relative integrable structures originally used by Voisin [43] is given by Proposition 2.12. We also state a general existence result for relative integrable structures (Proposition 2.13), which is proved in Section 7. In Section 2.3, we recall the construction of almost-complex Hilbert schemes only as topological spaces. For the construction of their differentiable structures, we refer the reader to Voisin's papers [44, Section 2] for an outline and [43, Section 3] for a detailed exposition. Then we state the main results of $[43 ; 44 ; 21]$ about almost-complex 
and symplectic Hilbert schemes. In Section 2.4, we recall briefly the construction of incidence varieties carried out in [21, Section 4], mainly to fix the notation. The compatibility conditions (A) and (B) introduced in Section 2.4 will appear several times in Section 3.5, Section 5.2 and Section 6.

In Section 3, we present the general theory of relatively coherent sheaves on the relative analytic spaces introduced in Section 2.2. This formalism is rather heavy and will be used in Section 5.2 to construct virtual tautological Chern characters and in Section 6 to compute the complex cobordism class of almost-complex Hilbert schemes. The reader interested only in the description of the cohomology ring of $X^{[n]}$ when $X$ is a symplectic compact four-manifold with vanishing first Betti number may skip this section except for Definition 3.1, and go directly to Section 4. In Section 3.1, we define relatively coherent sheaves as well as related operations: pullback, internal Hom, tensor product, and the corresponding derived operations. In Section 3.2, we define relative analytic subspaces and prove in Proposition 3.11 that their structure sheaves are relatively coherent. The proof relies on deep properties concerning ideals of differentiable functions. We also prove in Proposition 3.13 that relatively coherent sheaves are stable under pushforward by finite maps. The aim of Section 3.3 is to generalize the formalism of analytic $K$-theory of Borel and Serre [5] for relative analytic spaces. Suitable morphisms are introduced in Definition 3.14, so that the relatively coherent sheaves on a relative analytic space $\mathfrak{X}$ become an abelian category. The associated Grothendieck group is by definition the relative analytic $K$-theory of $\mathfrak{X}$. Then the operations defined in Section 3.2 and Section 3.3 induce operations in relative $K$-theory. Various formulae relating these operations are grouped in Proposition 3.16; they will be frequently used in Section 3.5, Section 5.2 and Section 6. The aim of Section 3.4 is to construct a map from relative analytic $K$-theory to topological $K$-theory with complex coefficients. In the case of usual analytic $K$-theory, such a map can be obtained via global real analytic locally free resolutions of coherent analytic sheaves (see Atiyah and Hirzebruch [2, Proposition 2.6]). It is also possible to use differentiable resolutions instead of real analytic ones, as explained in Atiyah and Hirzebruch [3, Section 6]; this is the method we adopt in the case of relatively coherent sheaves. The important point is that, although differentiable coherent sheaves as introduced in [3, Definition 6.1] are defined by a global condition, it turns out that they can be characterized by a local condition: it is the object of Proposition 3.17. This allows us to prove that any relatively coherent sheaf $\mathcal{F}$ on a relative analytic space $\mathfrak{X}$ admits a finite locally free resolution over $\mathcal{C}_{\mathfrak{X}}^{\infty}$ in a neighbourhood of every compact subset of $\mathfrak{X}$ (Proposition 3.19), and then to associate with $\mathcal{F}$ a well defined element in topological $K$-theory, called the topological class of $\mathcal{F}$. Next, we prove two important results about this class, namely the functoriality by pullback (Proposition 3.20) and 
the homotopy invariance (Proposition 3.21). In Section 3.5 we apply the formalism of Sections 3.1-3.4 and associate with any almost-complex compact four-manifold $X$ various incidence sheaves on relative analytic spaces built from $X$ via suitable relative integrable complex structures. This defines the geometric setting which will be used independently in Section 5.2 and Section 6. The last result of the section (Proposition 3.27), which is the analog of Ellingsrud, Göttsche and Lehn [14, Lemma 1.1] in a relative setting, will be used only in the proof of Proposition 6.8.

The object of Section 4 is to carry out for symplectic four-manifolds Lehn's computation of the boundary operator [25, Theorem 3.10]. In Section 4.1 we adapt the first part of Lehn's argument to the almost-complex case as we did for the Nakajima relations in [21]. This yields half of Lehn's formula (Theorem 4.2). In Section 4.2, we use Donaldson's theorem on symplectic divisors [11] to establish a general result (Proposition 4.6) concerning pseudoholomorphic curves on symplectic four-manifolds. This result allows us to obtain in Section 4.3 the other half of Lehn's formula when $X$ is symplectic: this is the object of Theorem 4.7 .

In Section 5, we deal with the cohomology rings of Hilbert schemes of points for symplectic compact four-manifolds. In Section 5.1, we prove an induction relation for the Chern characters of the tautological vector bundles constructed in [21] (Lemma 5.1). In Section 5.2, we construct virtual Chern characters satisfying the same induction relation (Proposition 5.2). As we already mentioned, we use the machinery of relatively coherent sheaves, and especially Section 3.5. Note that Section 5.2 can be skipped if the first Betti number of $X$ vanishes. In Section 5.3, we state and prove our main results about the cohomology rings of symplectic Hilbert schemes which are Theorems 5.6, 5.7 and 5.9.

Finally, Section 6 is entirely devoted to the computation of the cobordism class of Hilbert schemes of an almost-complex compact four-manifold (Theorem 6.1). We combine the strategy of [14, Section 1-3] with the use of the relative incidence sheaves of Section 3.5. In Sections 6.1, 6.2 and 6.3 respectively, we extend to the relative setting the results of [14, Propositions 2.2, 2.3 and 3.1]. This is the object of Propositions 6.4, 6.5 and 6.8 .

Acknowledgements I wish to thank my advisor Claire Voisin, whose work on almostcomplex Hilbert schemes is at the origin of this article. Her deep knowledge of the subject and her frequent and judicious advice have been most valuable to me. I also wish to thank her for her kindness and her patience. Finally, I thank the referee for his/her useful comments. 


\section{Almost-complex Hilbert schemes and relative analytic spaces}

\subsection{Hilbert schemes, incidence varieties and tautological bundles}

Let $X$ be a smooth complex manifold, $\mathcal{O}_{X}$ be its sheaf of holomorphic functions and $n$ be a positive integer.

Definition 2.1 The Hilbert-Douady scheme $X^{[n]}$ of $n$-points in $X$ is the set of zerodimensional subschemes of length $n$ in $X$, ie the set of ideal sheaves $\mathcal{J}$ of $\mathcal{O}_{X}$ such that $\sum_{p \in X} \operatorname{dim}_{\mathbb{C}} \mathcal{O}_{p} / \mathcal{J}_{p}$ is equal to $n$.

Let $\mathfrak{S}_{n}$ be the symmetric group on $n$ symbols and let $X^{(n)}:=X^{n} / \mathfrak{S}_{n}$ denote the $n$-fold symmetric product of $X$.

Definition 2.2 The Hilbert-Chow morphism $\Gamma: X^{[n]} \longrightarrow X^{(n)}$ is defined by the formula $\Gamma(\xi)=\sum_{p \in X} l_{p}(\xi) p$, where $l_{p}(\xi)$ is the length of $\xi$ at $p$.

Some basic properties of Hilbert schemes of points are:

- $X^{[n]}$ is a complex analytic space and $\Gamma$ is a bimeromorphic map.

- If $X$ is compact, so is $X^{[n]}$.

- The fibers of $\Gamma$ are projective, and irreducible if $\operatorname{dim} X=2$ by Briançon [6].

- If $X$ is a complex curve, then $\Gamma$ is an isomorphism and $X^{[n]}$ is smooth.

- If $X$ is a complex surface, then $X^{[n]}$ is smooth of dimension $2 n$ and is irreducible if $X$ is connected by Fogarty [16].

For a thorough study of Hilbert schemes of points, we refer the reader to Göttsche [18] and Nakajima [34].

In this section, we only consider the case $\operatorname{dim} X=2$. The Hilbert schemes corresponding to different values of $n$ are related through the incidence varieties:

Definition 2.3 For all positive integers $m$ and $n$ such that $m>n$, the incidence variety $X^{[m, n]}$ is the set of couples $\left(\xi, \xi^{\prime}\right)$ in $X^{[n]} \times X^{[m]}$ such that $\xi$ is a subscheme of $\xi^{\prime}$.

The incidence varieties $X^{[m, n]}$ are analytic subvarieties of $X^{[n]} \times X^{[m]}$. The case $m=n+1$ appears as particularly interesting in the theory:

- $X^{[n+1, n]}$ is smooth and irreducible by Cheah [8], Tikhomirov [41] and unpublished work of Ellingsrud. 
- If $Y_{n}$ is the incidence locus, defined as

$$
Y_{n}=\left\{(\xi, x) \text { in } X^{[n]} \times X \text { such that } x \in \operatorname{supp}(\xi)\right\},
$$

then $X^{[n+1, n]} \simeq \mathbb{P}\left(\mathcal{J}_{n}\right)$ by Danila [10, Proposition 3.3]. As a consequence, $X^{[n+1, n]}$ is isomorphic to the schematic blowup of $Y_{n}$ in $X^{[n]} \times X$. The exceptional divisor associated with this blowup is

$$
D=\left\{\left(\xi, \xi^{\prime}\right) \text { in } X^{[n+1, n]} \operatorname{such} \text { that } \operatorname{supp}(\xi)=\operatorname{supp}\left(\xi^{\prime}\right)\right\} .
$$

These properties show that the incidence variety $X^{[n+1, n]}$ is closely related to the incidence locus $Y_{n}$. The latter satisfies the following properties:

- The morphism $\left.\operatorname{pr}_{1}\right|_{Y_{n}}: Y_{n} \longrightarrow X^{[n]}$ is flat and finite.

- The ideal sheaf $\mathcal{J}_{Y_{n}}$ admits a global locally free resolution of length 2 on $X^{[n]} \times X$ by Danila [10, Lemma 3.2].

- If $\phi: X^{[n+1, n]} \longrightarrow X^{[n]}$ and $\psi: X^{[n+1, n]} \longrightarrow X^{[n+1]}$ are the natural morphisms induced by the projections, if $\rho: X^{[n+1, n]} \longrightarrow X$ is the residual map defined by the formula $\rho\left(\xi, \xi^{\prime}\right)=\operatorname{supp}\left(\xi / \xi^{\prime}\right)$, if $j=($ id, $\rho): X^{[n+1, n]} \longrightarrow X^{[n+1, n]} \times X$ and if $\mathcal{L}=\mathcal{O}_{X}{ }^{[n+1, n]}(-D)$, then there exists a natural exact sequence on the product $X^{[n+1, n]} \times X[14$, Section 1]

$$
0 \longrightarrow j_{*} \mathcal{L} \longrightarrow(\psi, \mathrm{id})^{*} \mathcal{O}_{Y_{n+1}} \longrightarrow(\phi, \mathrm{id})^{*} \mathcal{O}_{Y_{n}} \longrightarrow 0 \text {. }
$$

The variety $X^{[n+1, n]}$ can be constructed explicitly via a global locally free resolution

$$
0 \longrightarrow \mathcal{A} \longrightarrow \mathcal{B} \longrightarrow \mathcal{J}_{Y_{n}} \longrightarrow 0
$$

of $\mathcal{J}_{Y_{n}}:$ if $\mathbb{P}(\mathcal{B})$ is the projective bundle of $\mathcal{B}$ (using Grothendieck's convention for projective bundles), if $\pi: \mathbb{P}(\mathcal{B}) \longrightarrow X^{[n]} \times X$ is the associated projection and if $s$ is the section of $\pi^{*} \mathcal{A}^{*}(1)$ given by the morphism $\pi^{*} \mathcal{A} \rightarrow \pi^{*} \mathcal{B} \rightarrow \mathcal{O}_{\mathcal{B}}(1)$, then $s$ is transverse to the zero section and its vanishing locus is isomorphic to $X^{[n+1, n]}$.

We end this section with tautological bundles. Let $\mathrm{pr}_{1}$ and $\mathrm{pr}_{2}$ be the projections from $X^{[n]} \times X$ on the first and second factors.

Definition 2.4 Let $E$ be a holomorphic vector bundle on $X$ and $n$ be a positive integer. The tautological vector bundle $E^{[n]}$ is a holomorphic vector bundle on $X^{[n]}$ defined by the formula $E^{[n]}=\operatorname{pr}_{1 *}\left(\mathcal{O}_{Y_{n}} \cdot \operatorname{pr}_{2}^{*} E\right)$. 
If $E$ is a holomorphic vector bundle on $X$ and $n$ is a positive integer, the tautological vector bundles $E^{[n]}$ and $E^{[n+1]}$ are related through an exact sequence on $X^{[n+1, n]}$ (see [25, page 193]):

$$
0 \longrightarrow \rho^{*} E \otimes \mathcal{L} \longrightarrow \psi^{*} E^{[n+1]} \longrightarrow \phi^{*} E^{[n]} \longrightarrow 0 .
$$

\subsection{Relative spaces and relative integrable complex structures}

The geometric structure underlying the construction of the almost-complex Hilbert scheme of Voisin [43] is that of relative integrable structure, or in an almost equivalent way that of relative analytic space. In this article, this point of view is systematically expanded in order to study coherent sheaves in relative analytic spaces, which is done in Section 3.

Throughout this section, $B$ and $B^{\prime}$ denote compact differentiable effective orbifolds (see Satake [39] and Adem, Leida and Ruan [1, Section 1.1]). Recall that a map $f: B \longrightarrow B^{\prime}$ is smooth if for any $b$ in $B$ there exist two orbifold charts $\left(V_{b}, G_{b}\right.$, $\left.U_{b}\right)$ and $\left(V_{f(b)}, G_{f(b)}, U_{f(b)}\right)$ near $b$ and $f(b)$, a group morphism $\lambda: G_{b} \longrightarrow G_{f(b)}$ and a smooth $\lambda$-equivariant map from $V_{b}$ to $V_{f(b)}$ inducing $f$ on $U_{b}$.

Definition 2.5 Let $\mathfrak{X}$ be a separated topological space and $\pi: \mathfrak{X} \longrightarrow B$ be a continuous surjective map.

(i) A relative chart (resp. relative holomorphic chart) on $\mathfrak{X}$ is given by a homeomorphism $\phi: U \stackrel{\sim}{\longrightarrow} Y \times V$ such that $\left.\pi\right|_{U}=\operatorname{pr}_{2} \circ \phi$, where $U$ is an open subset of $\mathfrak{X}, V$ is an open subset of $B$ and $Y$ is a differentiable manifold (resp. $Y$ is a smooth analytic space).

(ii) Let $\phi: U \stackrel{\sim}{\longrightarrow} Y \times V$ and $\psi: U^{\prime} \stackrel{\sim}{\longrightarrow} Y^{\prime} \times V^{\prime}$ be two relative charts (resp. relative holomorphic charts) and let $(y, v) \longmapsto(\gamma(y, v), v)$ be the associated transition function $\psi \circ \phi^{-1}: \phi\left(U \cap U^{\prime}\right) \longrightarrow \psi\left(U \cap U^{\prime}\right)$. The charts $\phi$ and $\psi$ are compatible if $\gamma$ is smooth (resp. $\gamma$ is smooth and for all $v$ in $\operatorname{pr}_{2}\left(\phi\left(U \cap U^{\prime}\right)\right.$ ) the function $y \longmapsto \gamma(y, v)$ is holomorphic).

(iii) A relative atlas (resp. relative holomorphic atlas) on $\mathfrak{X}$ is a collection of compatible relative charts (resp. relative holomorphic charts) on $X$ whose domains cover $\mathfrak{X}$.

(iv) A relative atlas (resp. relative holomorphic atlas) $\mathcal{A}$ on $\mathfrak{X}$ is maximal if every relative chart (resp. relative holomorphic chart) on $\mathfrak{X}$ compatible with all the charts of $\mathcal{A}$ belongs to $\mathcal{A}$.

(v) If $\mathcal{A}$ is a relative atlas (resp. relative holomorphic atlas) on $\mathfrak{X}$, the saturated atlas of $\mathcal{A}$ is the smallest maximal atlas containing $\mathcal{A}$. 
To be able to define and study relatively coherent sheaves in Section 3, we introduce the notion of complete relative holomorphic atlas (cf Remark 3.3 (iii)).

Definition 2.6 Let $\mathfrak{X}$ be a separated topological space and let $\pi: \mathfrak{X} \longrightarrow B$ be a continuous surjective map.

(i) If $\phi: U \stackrel{\sim}{\longrightarrow} Y \times V$ is a relative holomorphic chart, if $Y^{\prime}$ (resp. $V^{\prime}$ ) is an open subset of $Y$ (resp. $V$ ) and if $U^{\prime}=\phi^{-1}\left(Y^{\prime} \times V^{\prime}\right)$, then $\left.\phi\right|_{U^{\prime}}: U^{\prime} \stackrel{\sim}{\longrightarrow} Y^{\prime} \times V^{\prime}$ is a relative holomorphic chart called the restriction of $\phi$ to $U^{\prime}$.

(ii) A relative holomorphic atlas $\mathcal{A}$ on $\mathfrak{X}$ is complete if

- for all relative holomorphic chart $\phi$ in $\mathcal{A}$, all the restrictions of $\phi$ are in $\mathcal{A}$.

- for all finite family $\left\{\phi_{i}: U_{i} \stackrel{\sim}{\longrightarrow} Y_{i} \times V_{i}\right\}_{1 \leq i \leq r}$ of relative holomorphic charts in $\mathcal{A}$ such that the open sets $U_{i}$ are pairwise disjoint, then the relative holomorphic chart $\coprod_{i=1}^{r} \phi_{i}: \amalg U_{i} \stackrel{\sim}{\longrightarrow} \amalg\left(Y_{i} \times V_{i}\right)$ is in $\mathcal{A}$.

(iii) If $\mathcal{A}$ is a relative holomorphic atlas on $\mathfrak{X}$, the completed atlas of $\mathcal{A}$ is the smallest complete relative holomorphic atlas containing $\mathcal{A}$.

(iv) If $\mathcal{A}$ and $\mathcal{A}^{\prime}$ are two relative holomorphic atlases on $\mathfrak{X}$, we say $\mathcal{A}$ refines $\mathcal{A}^{\prime}$ if for any relative holomorphic chart $\phi: U \stackrel{\sim}{\longrightarrow} Y \times V$ in $\mathcal{A}^{\prime}$ and any $x$ in $U$, there exists a neighbourhood $U_{x}$ of $x$ in $U$ such that $\left.\phi\right|_{U_{x}}$ is in $\mathcal{A}$.

(v) Two relative holomorphic atlases $\mathcal{A}$ and $\mathcal{A}^{\prime}$ are equivalent if $\mathcal{A}$ refines $\mathcal{A}^{\prime}$ and $\mathcal{A}^{\prime}$ refines $\mathcal{A}$.

We define now relative differentiable spaces and relative analytic spaces.

Definition 2.7 (i) A relative differentiable space (resp. relative analytic space) over $B$ is the data of a separated topological space $\mathfrak{X}$ endowed with a continuous surjective map $\pi: \mathfrak{X} \longrightarrow B$ and with a maximal relative atlas (resp. an equivalent class of complete relative holomorphic atlas) over $B$.

(ii) If $\mathfrak{X}$ is a separated topological space, if $\pi: \mathfrak{X} \longrightarrow B$ is a continuous surjective map and if $\mathcal{A}$ is a maximal relative atlas (resp. a complete relative holomorphic atlas) on $\mathfrak{X}$, we denote by $(\mathfrak{X}, \mathcal{A})$ the associated relative differentiable space (resp. relative analytic space).

Remark 2.8 (i) If $(\mathfrak{X}, \mathcal{A})$ is a relative differentiable space (resp. relative analytic space) over $B$, the fibers $\left(\mathfrak{X}_{b}\right)_{b \in B}$ defined by $\mathfrak{X}_{b}:=\pi^{-1}(b)$ are differentiable (resp. complex) manifolds, but they do not form in general a fibration over $B$, since the projection map $\pi$ is not assumed to be proper. 
(ii) The connected components of a relative differentiable space (resp. relative analytic space) $(\mathfrak{X}, \mathcal{A})$ are still relative differentiable spaces (resp. relative analytic spaces). If $\mathfrak{X}$ is connected, the dimension (resp. complex dimension) of $\mathfrak{X}_{b}$ is independent of $b$. We call it the relative real dimension (resp. relative complex dimension) of $\mathfrak{X}$.

(iii) If $(\mathfrak{X}, \mathcal{A})$ is a relative differentiable space over $B$, then $\mathfrak{X}$ is a differentiable orbifold and the projection $\pi: \mathfrak{X} \longrightarrow B$ is smooth.

Let us introduce some natural operations on relative analytic spaces.

Definition 2.9 (i) (Base change) Let $(\mathfrak{X}, \mathcal{A})$ be a relative differentiable space (resp. relative analytic space) over $B$ and $u: B^{\prime} \longrightarrow B$ be a smooth map. If $\mathcal{A}=$ $\left\{\phi_{i}: U_{i} \stackrel{\sim}{\longrightarrow} Y_{i} \times V_{i}\right\}_{i \in I}$, if $\mathcal{A}^{\prime}=\left\{\phi_{i} \times_{B} \text { id }_{B^{\prime}}: U_{i} \times{ }_{B} B^{\prime} \stackrel{\sim}{\longrightarrow} Y_{i} \times u^{-1}\left(V_{i}\right)\right\}_{i \in I}$ and if $u^{*}(\mathcal{A})$ is the saturated (resp. completed) atlas of $\mathcal{A}^{\prime}$, then $\left(\mathfrak{X} \times{ }_{B} B^{\prime}, u^{*}(\mathcal{A})\right)$ is a relative differentiable space (resp. relative analytic space) over $B^{\prime}$.

(ii) (Fiber product) Let $(\mathfrak{X}, \mathcal{A})$ and $\left(\mathfrak{X}^{\prime}, \mathcal{A}^{\prime}\right)$ be two relative differentiable spaces (resp. relative analytic spaces) over the same base $B$. Suppose $\phi: U \stackrel{\sim}{\longrightarrow} Z \times V$ and $\phi^{\prime}: U^{\prime} \stackrel{\sim}{\longrightarrow} Z^{\prime} \times V$ are two relative charts (resp. relative holomorphic charts) over the same open set $V$. Then $\phi \times{ }_{B} \phi^{\prime}: U \times{ }_{B} U^{\prime} \stackrel{\sim}{\longrightarrow}\left(Z \times Z^{\prime}\right) \times V$ is a relative chart (resp. relative holomorphic chart) on $\mathfrak{X} \times{ }_{B} \mathfrak{X}^{\prime}$. If $\mathcal{A}^{\prime \prime}$ is the relative atlas (resp. relative holomorphic atlas) on $\mathfrak{X} \times{ }_{B} \mathfrak{X}^{\prime}$ consisting of such charts $\phi \times_{B} \phi^{\prime}$ and if $\mathcal{A} \times{ }_{B} \mathcal{A}^{\prime}$ is the saturated (resp. completed) atlas of $\mathcal{A}^{\prime \prime}$, then $\left(\mathfrak{X} \times{ }_{B} \mathfrak{X}^{\prime}, \mathcal{A} \times{ }_{B} \mathcal{A}^{\prime}\right)$ is a relative differentiable space (resp. relative analytic space).

(iii) (Relative tangent bundle) Let $(\mathfrak{X}, \mathcal{A})$ be a relative differentiable space (resp. relative analytic space) over $B$, where $\mathcal{A}=\left\{\phi_{i}: U_{i} \stackrel{\sim}{\longrightarrow} Y_{i} \times V_{i}\right\}_{i \in I}$. We define a set

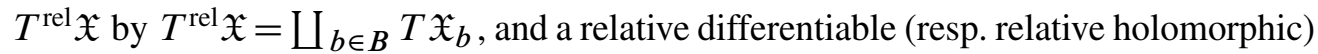
atlas $\mathcal{A}^{\prime}$ on $T^{\text {rel }} \mathfrak{X}$ by $\mathcal{A}^{\prime}=\left\{d^{\text {rel }} \phi_{i}: T^{\text {rel }} U_{i} \stackrel{\sim}{\longrightarrow} T Y_{i} \times V_{i}\right\}_{i \in I}$, where $d^{\text {rel }} \phi_{i}$ is the relative differential of $\phi_{i}$. If $T^{\text {rel }} \mathcal{A}$ is the saturated (resp. completed) atlas of $\mathcal{A}^{\prime}$, then $\left(T^{\text {rel }} \mathfrak{X}, T^{\text {rel }} \mathcal{A}\right.$ ) is a relative differentiable space (resp. relative analytic space) called the relative tangent bundle of $\mathfrak{X}$. As a topological space, $T^{\text {rel } \mathfrak{X}}$ is a topological vector bundle over $\mathfrak{X}$.

Relative analytic spaces are introduced by Voisin [43] by means of relative integrable structures:

Definition 2.10 Let $\mathfrak{X}$ be a relative differentiable space over $B$. A relative integrable complex structure $J^{\text {rel }}$ on $\mathfrak{X}$ is a continuous section of End $\left(T^{\text {rel } \mathfrak{X})}\right.$ satisfying the following conditions: 
- If $\phi: U \stackrel{\sim}{\longrightarrow} Y \times V$ is any relative chart of $\mathfrak{X}$, the map $J^{\text {rel }}: Y \times V \longrightarrow T Y$ is smooth.

- For every $b$ in $B$, the map $J_{b}^{\text {rel. }}: \mathfrak{X}_{b} \longrightarrow$ End $\left(T \mathfrak{X}_{b}\right)$ defines an integrable complex structure on $\mathfrak{X}_{b}$.

The next proposition allows us to construct local holomorphic trivializations for relative integrable complex structures.

Proposition 2.11 Let $(\mathfrak{X}, \mathcal{A})$ be a relative differentiable space over $B$ and $J^{\text {rel }}$ be a relative integrable complex structure on $\mathfrak{X}$. For every $x$ in $\mathfrak{X}$, there exist a neighbourhood $U_{x}$ of $x$ and a relative chart $\phi: U_{x} \stackrel{\sim}{\longrightarrow} W \times V$ in $\mathcal{A}$ such that:

- $W$ is an open subset of $\mathbb{C}^{N}$, where $2 N$ is the relative real dimension of the connected component of $x$ in $\mathfrak{X}$.

- If $J_{\text {st }}$ is the standard complex structure on $\mathbb{C}^{N}$ and if $b$ is any point in $V$, then

$$
\phi_{b}:\left(U_{x} \cap \mathfrak{X}_{b}, J_{b}^{\text {rel }}\right) \longrightarrow\left(W, J_{\text {st }}\right)
$$

is a biholomorphism.

Proof Let $\tilde{U}_{x}$ be a neighbourhood of $x, \widetilde{\phi}: \tilde{U}_{x} \stackrel{\sim}{\longrightarrow} Y \times V$ be a relative chart, and put $\tilde{\phi}(x)=\left(y_{0}, v_{0}\right)$. The relative integrable complex structure $J^{\text {rel }}$ defines a smooth family $\left(J_{v}\right)_{v \in V}$ of integrable complex structures on $Y$. By the Newlander-Nirenberg theorem with parameters, there exist a neighbourhood $U_{y_{0}} \times U_{v_{0}}$ of $\left(y_{0}, v_{0}\right)$ and smooth complex-valued functions $z^{1}, \ldots, z^{N}$ on $U_{y_{0}} \times U_{v_{0}}$ such that for every $v$ in $U_{v_{0}},\left(z_{v}^{1}, \ldots, z_{v}^{N}\right)$ are holomorphic coordinates on $\left(U_{y_{0}}, J_{v}\right)$ (see [44, page 271]). If $U_{x}=\widetilde{\phi}^{-1}\left(U_{y_{0}} \times U_{v_{0}}\right)$, we define $\phi$ on $U_{x}$ by $\phi=\left(\left(z^{1}, \ldots, z^{N}\right) \circ \widetilde{\phi}, \pi\right)$.

Proposition 2.11 enables us to relate relative complex structures and relative analytic spaces.

Proposition 2.12 Let $(\mathfrak{X}, \mathcal{A})$ be a relative differentiable space over $B$. There is a natural bijection between relative integrable complex structures on $\mathfrak{X}$ and maximal relative holomorphic atlases contained in $\mathcal{A}$.

Proof If $\mathfrak{X}$ is endowed with a structure of relative analytic space over $B$, Proposition 2.11 allows us to construct a relative holomorphic atlas on $\mathfrak{X}$ and then the corresponding saturated atlas. Conversely, if $\left\{\phi_{i}: U_{i} \stackrel{\sim}{\longrightarrow} Z_{i} \times V_{i}\right\}_{i \in I}$ is a relative holomorphic atlas on $\mathfrak{X}$, the complex structures of the $Z_{i}$ 's define a relative integrable complex structure on $\mathfrak{X}$.

As a corollary, if $\mathfrak{X}$ is a relative analytic space over $B$, there exists a canonical relative integrable complex structure on the underlying relative differentiable space. 
Relative integrable structures are introduced in [43] to deal with problems in almostcomplex geometry. Let us give some general results about relative integrable complex structures associated with an almost-complex manifold.

Let $(X, J)$ be an almost-complex compact manifold and $B$ be a compact connected differentiable orbifold. Recall that $B$ carries a stratification with finitely many strata. If $Z$ is a closed subset of $X \times B$, we say that $Z$ is an incidence set if the following conditions are satisfied:

- The map $\operatorname{pr}_{2} \mid Z: Z \longrightarrow B$ is surjective and finite.

- For each stratum $B_{\lambda}$ of $B$, if $Z_{\lambda}=\operatorname{pr}_{2}^{-1}\left(B_{\lambda}\right) \cap Z$, then $Z_{\lambda}$ is a submanifold of $X \times B_{\lambda}$ and the map $\left.\operatorname{pr}_{2}\right|_{Z_{\lambda}}: Z_{\lambda} \longrightarrow B_{\lambda}$ is a covering map.

Let us introduce now some notation. Let $g$ be a Riemannian metric on $X$, $\varepsilon$ be a positive integer and $Z$ be an incidence set of $X \times B$.

- If $W$ is neighbourhood of $Z$ in $X \times B$ (considered as a relative differentiable space over $B), \mathcal{B}_{g, \varepsilon}(W)$ will denote the set of relative integrable complex structures $J^{\text {rel }}$ on $W$ such that $\left\|J^{\text {rel }}-J\right\|_{C^{0}, g, W}<\varepsilon$.

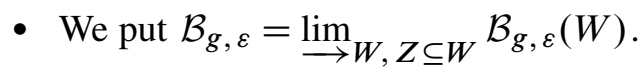

Proposition 2.13 Let $B$ be a compact differentiable orbifold, $(X, J)$ be an almostcomplex compact manifold and $Z$ be an incidence set in $X \times B$. If $g$ is a Riemannian metric on $X$, there exists a positive real number $\varepsilon_{0}$ such that for any positive $\varepsilon$ smaller than $\varepsilon_{0}, \mathcal{B}_{g, \varepsilon}$ is nonempty and weakly contractible in the following sense: for every nonnegative integer $p$ and every pair of smooth families $\left(J_{0, s}^{\mathrm{rel}}\right)_{s \in \mathbb{S}^{p}}$ and $\left(J_{1, s}^{\mathrm{rel}}\right)_{s \in \mathbb{S} p}$ in $\mathcal{B}_{g, \varepsilon}$ parameterized by the sphere $\mathbb{S}^{p}$, there exists a smooth family $\left(J_{t, s}^{\mathrm{rel}}\right)_{(t, s) \in[0,1] \times \mathbb{S}^{p}}$ parameterized by $[0,1] \times \mathbb{S}^{p}$ joining $\left(J_{0, s}^{\mathrm{rel}}\right)$ and $\left(J_{1, s}^{\mathrm{rel}}\right)$.

This result is implicit in [44], although not stated in this degree of generality (see [44, Proposition 4]). We provide a proof in Section 7.

\subsection{Construction of the almost-complex Hilbert scheme}

First of all, we define relative Hilbert schemes.

Definition 2.14 Let $(\mathfrak{X}, \mathcal{A})$ be a relative analytic space over $B$ of relative complex dimension two and $J^{\text {rel }}$ be the associated relative integrable complex structure. For any positive integer $n$, we define a set $\mathfrak{X}_{\text {rel }}^{[n]}$ by $\mathfrak{X}_{\text {rel }}^{[n]}=\bigsqcup_{b \in B} \mathfrak{X}_{b}^{[n]}$, where $\mathfrak{X}_{b}^{[n]}$ is the Hilbert scheme of $n$-points of $\mathfrak{X}_{b}$ endowed with the integrable complex structure $J_{b}^{\text {rel }}$. If

$$
\mathcal{A}=\left\{\phi_{i}: U_{i} \stackrel{\sim}{\longrightarrow} Y_{i} \times V_{i}\right\}_{i \in I} \quad \text { and } \quad \mathcal{A}^{\prime}=\left\{\phi_{i, \mathrm{rel}}^{[n]}: U_{i, \mathrm{rel}}^{[n]} \longrightarrow Y_{i}^{[n]} \times V_{i}\right\}_{i \in I}
$$


and if $\mathcal{A}^{[n]}$ is the completed atlas of $\mathcal{A}^{\prime}$, then $\left(\mathfrak{X}_{\text {rel }}^{[n]}, \mathcal{A}^{[n]}\right)$ is a relative analytic space called the relative Hilbert scheme of $\mathfrak{X}$.

Let us now recall the definition of Voisin's almost-complex Hilbert schemes. Let $(X, J)$ be an almost-complex compact manifold of dimension four, $g$ be a Riemannian metric on $X, n$ be a positive integer and $Z_{n}$ be the incidence set in $X \times X^{(n)}$ defined by

$$
Z_{n}=\left\{(p ; \underline{x}) \text { in } X \times X^{(n)} \text { such that } p \in \underline{x}\right\} .
$$

We use the notation introduced at the end of Section 2.2. Let $\varepsilon$ be any positive real number smaller than the bound $\varepsilon_{0}$ of Proposition 2.13. Then $\mathcal{B}_{g, \varepsilon}$ is nonempty.

Definition 2.15 Let $W$ be a small neighbourhood of $Z_{n}$ in $X \times X^{(n)}$ and $J^{\text {rel }}$ be a relative integrable complex structure in $\mathcal{B}_{g, \varepsilon}(W)$. The topological Hilbert scheme $X_{J^{\text {rel }}}^{[n]}$ is the subset of the relative Hilbert scheme $W_{\text {rel }}^{[n]}$ defined by

$$
X_{J \text { rel }}^{[n]}=\left\{(\xi ; \underline{x}) \text { in } W_{\text {rel }}^{[n]} \text { such that } \underline{x}=\Gamma_{\underline{x}}(\xi)\right\},
$$

where $\Gamma_{\underline{x}}: W_{\underline{x}}^{[n]} \longrightarrow W_{\underline{x}}^{(n)}$ is the Hilbert-Chow morphism associated with the integrable complex structure $J_{\underline{x}}^{\text {rel }}$.

Remark 2.16 (i) The topological Hilbert scheme $X_{J^{\text {rel }}}^{[n]}$ depends only on the germ of $J^{\text {rel }}$ along $Z_{n}$, ie of the image of $J^{\text {rel }}$ in $\mathcal{B}_{g, \varepsilon}$.

(ii) If $W$ is a small neighbourhood of $Z_{n}$, let $\left\{J_{t}^{\text {rel }}\right\}_{t \in B(0, r) \subseteq \mathbb{R}^{d}}$ be a smooth family in $\mathcal{B}_{g, \varepsilon}(W)$. This family defines a relative integrable complex structure $\widetilde{J}^{\text {rel }}$ on the relative analytic space $\widetilde{W}=W \times_{X^{(n)}}\left(X^{(n)} \times B(0, r)\right)$. Then there exists a natural relative topological Hilbert scheme over $B(0, r)$ whose fibers are the $\left(X_{J_{t}^{[\mathrm{rl}}}^{[n]}\right)_{t \in B(0, r)}$, namely,

$$
\left(X^{[n]},\left\{J_{t}^{\mathrm{rel}}\right\}_{t \in B(0, r)}\right)=\left\{(\xi ; \underline{x}, t) \text { in } \widetilde{W}_{\text {rel }}^{[n]} \text { such that } \underline{x}=\Gamma_{\underline{x}, t}(\xi)\right\},
$$

where $\Gamma_{\underline{x}, t}: W_{\underline{x}}^{[n]} \longrightarrow W_{\underline{x}}^{(n)}$ is the Hilbert-Chow morphism associated with the complex structure $\widetilde{J}_{\underline{x}, t}^{\text {rel }}$.

To obtain a differentiable structure on $X_{J^{\text {rel }}}^{[n]}$, Voisin uses relative integrable structures in a contractible subset $\mathcal{B}^{\prime}$ of $\mathcal{B}_{g, \varepsilon}$ satisfying some additional geometric conditions (see [43, page 711]). The main results she obtains are:

Theorem 2.17 [43, Theorem 5, Theorem 6, Theorem 3; 44, Theorem 3] Let $(X, J)$ be an almost-complex compact four-manifold, $J^{\text {rel }}$ be a relative integrable structure in $\mathcal{B}^{\prime}$ and $n$ be a positive integer. Then 
(i) $X_{J^{\text {rel }}}^{[n]}$ has a natural differentiable structure. Furthermore, if $J^{\prime \text { rel }}$ is another relative integrable structure in $\mathcal{B}^{\prime}$, there is a diffeomorphism between $X_{J^{\text {rel }}}^{[n]}$ and $X_{J^{\prime} \text { rel }}^{[n]}$ which is uniquely defined up to isotopy.

(ii) There is a canonical Hilbert-Chow map $\Gamma: X_{J \text { rel }}^{[n]} \longrightarrow X^{(n)}$ satisfying the following property: for any $\underline{x}$ in $X^{(n)}$ and any integrable complex structure in a neighbourhood $U_{\underline{x}}$ of $\operatorname{supp}(\underline{x}), \Gamma^{-1}(\underline{x})$ is homeomorphic to the fiber at $x$ of the usual Hilbert-Chow morphism from $U_{\underline{x}}^{[n]}$ to $U_{\underline{x}}^{(n)}$.

(iii) $X_{J_{\text {rel }}}^{[n]}$ can be endowed with a stable almost-complex structure, and the associated complex cobordism class of $X_{J^{\text {rel }}}^{[n]}$ depends only on the deformation class of $J$.

(iv) If $X$ is symplectic and $J$ is compatible with the symplectic structure, $X_{J^{\text {rel }}}^{[n]}$ is also symplectic.

For arbitrary relative integrable structures, this theorem has the following topological form:

Theorem 2.18 [21, Proposition 3.4, Proposition 3.10, Remark 3.5]

(i) Let $J^{\text {rel }}$ be a relative integrable complex structure in $\mathcal{B}_{g, \varepsilon}$. Then $X_{J \text { rel }}^{[n]}$ is a topological manifold of real dimension $4 n$.

(ii) If $W$ is a neighbourhood of $Z_{n}$ in $X \times X^{(n)}$ and if $\left\{J_{t}^{\text {rel }}\right\}_{t \in B(0, r) \subseteq \mathbb{R}^{d}}$ is a smooth path in $\mathcal{B}_{g, \varepsilon}(W)$, then the associated relative topological Hilbert scheme $\left(X^{[n]},\left\{J_{t}^{\mathrm{rel}}\right\}_{t \in B(0, r)}\right)$ over $B(0, r)$ is a topological fibration (cf Remark 2.16 (ii)).

(iii) For any $\underline{x}$ in $X^{(n)}$ and any integrable structure $J$ in a neighbourhood $U_{\underline{x}}$ of $\operatorname{supp}(\underline{x})$, the Hilbert-Chow morphism $\Gamma: X_{J_{\text {rel }}^{[n]}}^{\longrightarrow} X^{(n)}$ is locally homeomorphic over a neighbourhood of $\operatorname{supp}(\underline{x})$ to the classical Hilbert-Chow morphism from $U_{\underline{x}}^{[n]}$ to $U_{\underline{x}}^{(n)}$.

We can compare almost-complex Hilbert schemes corresponding to different relative integrable complex structures. Let $g$ and $g^{\prime}$ be two Riemannian metrics on $X, \varepsilon_{0}$ and $\varepsilon_{0}^{\prime}$ be the bounds given by Proposition 2.13, $\varepsilon$ and $\varepsilon^{\prime}$ be positive real numbers smaller than $\varepsilon_{0}$ and $\varepsilon_{0}^{\prime}$ respectively and $J^{\text {rel }}, J^{\prime \text { rel }}$ be relative integrable complex structures in $\mathcal{B}_{g, \varepsilon}$ and $\mathcal{B}_{g^{\prime}, \varepsilon^{\prime}}$. Then there exists a positive real number $\varepsilon^{\prime \prime}$ smaller than $\varepsilon$ such that $\mathcal{B}_{g, \varepsilon^{\prime \prime}}$ is included in $\mathcal{B}_{g^{\prime}, \varepsilon^{\prime}}$. Since, by Proposition $2.13, \mathcal{B}_{g, \varepsilon}, \mathcal{B}_{g^{\prime}, \varepsilon^{\prime}}$ and $\mathcal{B}_{g, \varepsilon^{\prime \prime}}$ are nonempty, connected and simply connected, $X_{J^{\text {rel }}}^{[n]}$ and $X_{J^{\prime r e l}}^{[n]}$ are homeomorphic by Theorem 2.18 (ii); and this homeomorphism is canonical up to isotopy.

Therefore, for every positive integer $n$, there exists a canonical ring $H^{*}\left(X^{[n]}, \mathbb{Q}\right)$ (resp. $K\left(X^{[n]}\right)$ ) such that for every relative integrable complex structure $J^{\text {rel }}$ in $\mathcal{B}_{g, \varepsilon}$, the 
$\operatorname{ring} H^{*}\left(X_{J^{\text {rel }}}^{[n]}, \mathbb{Q}\right)$ (resp. the ring $\left.K\left(X_{J^{\text {rel }}}^{[n]}\right)\right)$ is canonically isomorphic to $H^{*}\left(X^{[n]}, \mathbb{Q}\right)$ (resp. to $\left.K\left(X^{[n]}\right)\right)$.

Theorem 2.18 (iii) implies that Göttsche's classical formula for the Betti numbers of Hilbert schemes of points also holds in the almost-complex case (see [22, Theorem 3.9]).

\subsection{Incidence varieties and Nakajima operators}

If $m$ and $n$ are two positive integers, let

$$
Z_{n \times m}=\left\{(p ; \underline{x}, \underline{y}) \text { in } X \times X^{(n)} \times X^{(m)} \text { such that } p \in \underline{x} \cup \underline{y}\right\} .
$$

Relative integrable structures in a neighbourhood of $Z_{n \times m}$ are denoted by $J_{n \times m}^{\text {rel }}$.

Definition 2.19 (i) If $J_{1, n \times m}^{\text {rel }}$ and $J_{2, n \times m}^{\text {rel }}$ are two relative integrable structures in neighbourhoods $W_{1}$ and $W_{2}$ of $Z_{n \times m}$, then the product Hilbert scheme $\left(X^{[n] \times[m]}, J_{1, n \times m}^{\mathrm{rel}}, J_{2, n \times m}^{\mathrm{rel}}\right)$ is defined by

$$
\begin{aligned}
\left(X^{[n] \times[m]}, J_{1, n \times m}^{\mathrm{rel}}, J_{2, n \times m}^{\mathrm{rel}}\right)= & \left\{\left(\xi, \xi^{\prime} ; \underline{x}, \underline{z}\right) \text { in } W_{1, \text { rel }}^{[n]} \times_{X^{(n)} \times X^{(m)}} W_{2, \text { rel }}^{[m]}\right. \\
& \text { such that } \left.\Gamma_{1, \underline{x}, \underline{z}}(\xi)=\underline{x} \text { and } \Gamma_{2, \underline{x}, \underline{z}}\left(\xi^{\prime}\right)=\underline{z}\right\} .
\end{aligned}
$$

(ii) If $m>n$ and if $J_{n \times(m-n)}^{\mathrm{rel}}$ is a relative integrable structure in a neighbourhood $W$ of $Z_{n \times(m-n)}$, the incidence variety $\left(X^{[m, n]}, J_{n \times(m-n)}^{\text {rel }}\right)$ is defined by

$$
\begin{aligned}
\left(X^{[m, n]}, J_{n \times(m-n)}^{\mathrm{rel}}\right)= & \left\{\left(\xi, \xi^{\prime} ; \underline{x}, \underline{y}\right) \text { in } W_{\mathrm{rel}}^{[n]} \times_{X^{(n)} \times X^{(m-n)}} W_{\mathrm{rel}}^{[m]}\right. \\
& \text { such that } \left.\xi \subset \xi^{\prime}, \Gamma_{\underline{x}}, \underline{y}(\xi)=\underline{x} \text { and } \Gamma_{\underline{x}, \underline{y}}\left(\xi^{\prime}\right)=\underline{x} \cup \underline{y}\right\} .
\end{aligned}
$$

As it is the case for topological Hilbert schemes, the product Hilbert schemes and the incidence varieties are canonically defined up to homeomorphisms isotopic to the identity if the relative integrable structures used to define them are chosen close enough to $J$ in $C^{0}$-norm.

From now on, we fix a Riemannian metric $g$ on $X$ and assume that all relative integrable structures are sufficiently close to $J$ in $C^{0}$-norm.

Let $J_{n \times(m-n)}^{\text {rel }}, J_{n}^{\text {rel }}, J_{m}^{\text {rel }}$ and $J_{n \times m}^{\text {rel }}$ be relative integrable structures in neighbourhoods $W, W^{\prime}, W^{\prime \prime}$ and $\widetilde{W}$ of $Z_{n \times(m-n)}, Z_{n}, Z_{m}$ and $Z_{n \times m}$, respectively. We consider the following compatibility conditions of relative analytic spaces:

(A) For every $(\underline{x}, \underline{y})$ in $X^{(n)} \times X^{(m-n)}, W_{\underline{x}}^{\prime} \subseteq W_{\underline{x}, \underline{y}}$ and $\left.J_{n \times(m-n), \underline{x}, \underline{y}}^{\mathrm{rel}}\right|_{W_{\underline{x}}^{\prime}}=$ $J_{n, \underline{x}}^{\mathrm{rel}}$; ie $W^{\prime} \times X^{(n)}\left(X^{(n)} \times X^{(m-n)}\right) \subseteq W$, where the base change map is the first projection. If this condition holds, there is a natural morphism $\lambda$ from $\left(X^{[m, n]}, J_{n \times(m-n)}^{\mathrm{rel}}\right)$ to $\left(X^{[n]}, J_{n}^{\mathrm{rel}}\right)$. 
(B) For every $(\underline{x}, \underline{y})$ in $X^{(n)} \times X^{(m-n)}, W_{\underline{x}}^{\prime \prime} \cup y=W_{\underline{x}}, \underline{y}$ and $J_{n \times(m-n), \underline{x}, \underline{y}}^{\mathrm{rel}}=$ $J_{m, \underline{x} \cup y}^{\mathrm{rel}}$; ie $W^{\prime \prime} \times_{X^{(m)}}\left(X^{(n)} \times X^{(m-n)}\right) \stackrel{\underline{x}}{=} \underline{W}$, where the base change máp is $(\underline{x}, \underline{y}) \stackrel{\underline{x}}{\longmapsto} \cup \underline{y}$. If this condition holds, there is a canonical morphism $v$ from $\left(X^{[\bar{m}, n]}, J_{n \times(m-n)}^{\mathrm{rel}}\right)$ to $\left(X^{[m]}, J_{m}^{\mathrm{rel}}\right)$.

(C) For every $(\underline{x}, \underline{y})$ in $X^{(n)} \times X^{(m-n)}, \widetilde{W}_{x}, \underline{x} \cup \underline{y}=W_{\underline{x}, \underline{y}}$ and $J_{n \times(m-n), \underline{x}, \underline{y}}^{\mathrm{rel}}=$ $J_{n \times m, \underline{x}, \underline{x} \cup y}^{\mathrm{rel}}$; ie $\widetilde{W} \times{ }_{X^{(n)} \times X^{(m)}}\left(X^{(n)} \times X^{(m-n)}\right)=\underline{W}$, where the base change map is $(\underline{x}, \underline{y}) \longmapsto(\underline{x}, \underline{x} \cup \underline{y})$. If this condition holds, there is a natural embedding of $\left(X^{[m, n]}, J_{n \times(m-n)}^{\text {rel }}\right)$ into the product Hilbert scheme $\left(X^{[n] \times[m]}, J_{n \times m}^{\mathrm{rel}}, J_{n \times m}^{\mathrm{rel}}\right)$.

Each of these conditions can be satisfied for a suitable choice of relative integrable complex structures (this is obvious for conditions (B) and (C); for condition (A), it is necessary to use the gluing method developed in Section 7). Unfortunately, conditions (A) and (B) cannot hold at the same time, unless $X$ carries an integrable complex structure. Indeed, if $n=1$ and $m=2$, assume that we are given three relative integrable complex structures $J_{1 \times 1}^{\text {rel }}, J_{1}^{\text {rel }}$ and $J_{2}^{\text {rel }}$ such that $\left(J_{1}^{\text {rel }}, J_{1 \times 1}^{\text {rel }}\right)$ satisfies (A) and $\left(J_{2}^{\text {rel }}, J_{1 \times 1}^{\text {rel }}\right)$ satisfies (B). Then, for all $x$ and $y$ in $X$,

$$
\begin{aligned}
\left.J_{1, x}^{\mathrm{rel}}\right|_{W_{x}^{\prime} \cap W_{y}^{\prime}}=\left.J_{1 \times 1, x, y}^{\mathrm{rel}}\right|_{W_{x}^{\prime} \cap W_{y}^{\prime}} & =\left.J_{2,\{x, y\}}^{\mathrm{rel}}\right|_{W_{x}^{\prime} \cap W_{y}^{\prime}} \\
& =\left.J_{1 \times 1, y, x}^{\mathrm{rel}}\right|_{W_{x}^{\prime} \cap W_{y}^{\prime}}=\left.J_{1, y}^{\mathrm{rel}}\right|_{W_{x}^{\prime} \cap W_{y}^{\prime}}
\end{aligned}
$$

so that $J_{1}^{\text {rel }}$ defines a global integrable complex structure on $X$.

If $J_{n \times(m-n)}^{\text {rel }}$ is a relative integrable complex structure in a neighbourhood of $Z_{n \times(m-n)}$, let us fix four relative integrable complex structures $J_{n}^{\mathrm{rel}}, J_{1, n \times(m-n)}^{\mathrm{rel}}, J_{m}^{\mathrm{rel}}$ and $J_{2, n \times(m-n)}^{\text {rel }}$ in respective neighbourhoods of $Z_{n}, Z_{n \times(m-n)}, Z_{m}$ and $Z_{n \times(m-n)}$ such that $\left(J_{n}^{\text {rel }}, J_{1, n \times(m-n)}^{\text {rel }}\right)$ satisfies the compatibility condition (A) and $\left(J_{m}^{\text {rel }}, J_{2, n \times(m-n)}^{\text {rel }}\right)$ satisfies the compatibility condition (B). For $i=1,2$, there is a homeomorphism between $\left(X^{[m, n]}, J_{m \times(m-n)}^{\text {rel }}\right)$ and $\left(X^{[m, n]}, J_{i, m \times(m-n)}^{\text {rel }}\right)$ which is canonical up to isotopy. In this way, we get two continuous maps from $\left(X^{[m, n]}, J_{m \times(m-n)}^{\text {rel }}\right)$ to $\left(X_{J_{n}^{\text {rel }}}^{[n]}\right)$ and $\left(X_{J_{m}^{\text {rel }}}^{[m]}\right)$. Their homotopy classes are canonical and are still denoted by $\lambda$ and $v$.

The incidence varieties $X^{[m, n]}$ are locally homeomorphic to the integrable model $U^{[m, n]}$ where $U$ is an open set of $\mathbb{C}^{2}$; this allows us to put a stratification on each $X^{[m, n]}$. In this way, the $X^{[m, n]}$ are stratified topological spaces locally homeomorphic to analytic spaces endowed with their natural stratifications, and so each of them has a fundamental homology class.

The construction of representations of the Heisenberg superalgebra $\mathcal{H}\left(H^{*}(X, \mathbb{Q})\right)$ of $H^{*}(X, \mathbb{Q})$ into $\mathbb{H}=\bigoplus_{n \in \mathbb{N}} H^{*}\left(X^{[n]}, \mathbb{Q}\right)$ via correspondence actions of incidence varieties done by Nakajima [34] and Grojnowski [23] also holds in the almost-complex setting: 
Theorem 2.20 [21, Definition 4.3, Theorem 4.5] If $(X, J)$ is an almost-complex compact four-manifold, Nakajima operators $\left\{\mathfrak{q}_{i}(\alpha), i \in \mathbb{Z}, \alpha \in H^{*}(X, \mathbb{Q})\right\}$ can be constructed. They depend only on the deformation class of $J$ and satisfy the Heisenberg commutation relations:

$$
\forall i, j \in \mathbb{Z}, \forall \alpha, \beta \in H^{*}(X, \mathbb{Q}), \quad\left[\mathfrak{q}_{i}(\alpha), \mathfrak{q}_{j}(\beta)\right]=i \delta_{i+j, 0}\left(\int_{X} \alpha \beta\right) \mathrm{id}_{\mathbb{H}} .
$$

Furthermore, these operators induce an irreducible representation of $\mathcal{H}\left(H^{*}(X, \mathbb{Q})\right)$ in $\mathbb{H}$ with highest weight vector 1.

Finally, we introduce relative incidence varieties, which are essential in Sections 4.1, 5.2, 6.2 and 6.3.

Definition 2.21 If $m, n$ are two positive integers with $m>n$, if $W$ is a neighbourhood of $Z_{n \times(m-n)}$ and if $J_{n \times(m-n)}^{\text {rel }}$ is a relative integrable complex structure on $W, W_{\mathrm{rel}}^{[m, n]}$ is the subset of $W_{\mathrm{rel}}^{[n]} \times X^{(n)} \times X^{(m-n)} W_{\text {rel }}^{[m]}$ defined by

$$
W_{\mathrm{rel}}^{[m, n]}=\left\{\left(\xi, \xi^{\prime} ; \underline{x}, \underline{y}\right) \text { in } W_{\mathrm{rel}}^{[n]} \times_{X^{(n)} \times X^{(m-n)}} W_{\mathrm{rel}}^{[m]} \text { such that } \xi \subseteq \xi^{\prime}\right\} .
$$

If $\left\{\phi_{i}: U_{i} \stackrel{\sim}{\longrightarrow} \Omega_{i} \times V_{i}\right\}_{i \in I}$ is a maximal relative holomorphic atlas on $W$, then for all $i$ in $I$ we have $\left(\phi_{i}^{[n]} \times_{V_{i}} \phi_{i}^{[m]}\right)\left[\left(U_{i, \text { rel }}^{[n]} \times_{V_{i}} U_{i, \text { rel }}^{[m]}\right) \cap W_{\text {rel }}^{[m, n]}\right]=\Omega_{i}^{[m, n]} \times V_{i}$. Therefore, for $m=n+1$, the relative incidence varieties $W_{\text {rel }}^{[m, n]}$ are relative analytic spaces.

\section{Coherent sheaves on relative analytic spaces}

In this part, the letters $B$ and $B^{\prime}$ always denote compact differentiable effective orbifolds.

\subsection{Operations on relatively coherent sheaves}

We start by defining relative holomorphic functions.

Definition 3.1 Let $(\mathfrak{X}, \mathcal{A})$ be a relative analytic space over $B$. A continuous complexvalued function $f$ defined on an open subset $\Omega$ of $\mathfrak{X}$ is relatively holomorphic if for any $x$ in $\Omega$ and for any relative holomorphic chart $\phi: U \stackrel{\sim}{\longrightarrow} Z \times V$ of $\mathcal{A}$ in a neighbourhood of $x$, the function $f \circ \phi^{-1}$ is smooth and holomorphic in the variables of $Z$ in a neighbourhood of $\phi(x)$.

The sheaf $\mathcal{O}_{\mathfrak{X}}^{\text {rel }}$ of relatively holomorphic functions on $\mathfrak{X}$ is a sheaf of rings on $\mathfrak{X}$. 
Definition 3.2 Let $(\mathfrak{X}, \mathcal{A})$ be a relative analytic space over $B$. A sheaf $\mathcal{F}$ of $\mathcal{O}_{\mathfrak{X}}^{\text {rel }}$ modules is relatively coherent if there exists a relative holomorphic atlas $\widetilde{\mathcal{A}}$ equivalent to $\mathcal{A}$ such that for any relative holomorphic chart $\phi: U \stackrel{\sim}{\longrightarrow} Z \times V$ in $\widetilde{\mathcal{A}}$, there exists a coherent analytic sheaf $\overline{\mathcal{F}}$ on $Z$ such that $\left.\mathcal{F}\right|_{U}$ and $\phi^{-1}\left(\operatorname{pr}_{1}^{-1} \overline{\mathcal{F}} \otimes_{\mathrm{pr}_{1}^{-1} \mathcal{O}_{Z}} \mathcal{O}_{Z \times V}^{\text {rel }}\right)$ are isomorphic as sheaves of $\mathcal{O}_{Z \times V}^{\text {rel }}$-modules.

An equivalent definition of relatively coherent sheaves can be stated using gluing conditions: if $\tilde{\mathcal{A}}=\left\{\phi_{i}: U_{i} \stackrel{\sim}{\longrightarrow} Z_{i} \times V_{i}\right\}_{i \in I}$ and if $\phi_{i j}:=\phi_{i} \circ \phi_{j}^{-1}$ are the associated transition functions, a relatively coherent sheaf on $\mathfrak{X}$ is given by a family of coherent sheaves $\left\{\overline{\mathcal{F}}_{i}\right\}_{i \in I}$ on the smooth analytic sets $\left\{Z_{i}\right\}_{i \in I}$ and a family of isomorphisms of sheaves of $\mathcal{O}_{\phi_{j}\left(U_{i j}\right)}^{\text {rel }}$-modules between $\phi_{i j}^{-1}\left[\left.\left(\overline{\mathcal{F}}_{i} \otimes_{\mathrm{pr}_{1}^{-1} \mathcal{O}_{Z_{i}}} \mathcal{O}_{Z_{i} \times V_{i}}^{\text {rel }}\right)\right|_{\phi_{i}\left(U_{i j}\right)}\right]$ and

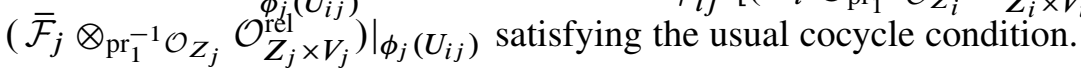

Remark 3.3 (i) Let $\mathcal{F}$ be a relatively coherent sheaf on $\mathfrak{X}$ given by a family of sheaves $\left\{\overline{\mathcal{F}}_{i}\right\}_{i \in I}$. Then, for any $b$ in $B$, if $J$ is the set of the indices $i$ in $I$ such that $b$ belongs to $V_{i}$, the sheaves $\left\{\overline{\mathcal{F}}_{i}\right\}_{i \in J}$ on $\left\{Z_{i} \times b\right\}_{i \in J}$ patch together into a coherent analytic sheaf on $\mathfrak{X}_{b}$, which we denote by $\mathcal{F}_{b}$.

(ii) If $(\mathfrak{X}, \mathcal{A})$ is a relative analytic space and if $\mathcal{E}$ is a locally-free sheaf of $\mathcal{O}_{\mathfrak{X}}^{\text {rel }}$ modules, then $\mathcal{E}$ is relatively coherent. In particular $T^{\text {rel } \mathfrak{X}}$ is relatively coherent on $\mathfrak{X}$.

(iii) If $(\mathfrak{X}, \mathcal{A})$ is a maximal relative analytic space, it is not difficult to prove that any relatively coherent sheaf on $\mathfrak{X}$ is in fact locally $\mathcal{O}_{\mathfrak{X}}$-free. This fact justifies the use of nonmaximal atlases.

Let us now introduce a class of morphisms between relative analytic spaces that is well-adapted to relatively coherent sheaves.

Definition 3.4 (i) Let $(\mathfrak{X}, \mathcal{A})$ and $\left(\mathfrak{X}^{\prime}, \mathcal{A}^{\prime}\right)$ be two relative analytic spaces over $B$ and let $f: \mathfrak{X} \longrightarrow \mathfrak{X}^{\prime}$ be a continuous map over $B$. Two relative holomorphic charts $\phi: U \stackrel{\sim}{\longrightarrow} Z \times V$ and $\phi^{\prime}: U^{\prime} \stackrel{\sim}{\longrightarrow} Z^{\prime} \times V$ in $\mathcal{A}$ and $\mathcal{A}^{\prime}$ are $f-$ compatible if $f(U) \subseteq U^{\prime}$ and if there exists a holomorphic map $g: Z \longrightarrow Z^{\prime}$ such that the following diagram commutes:

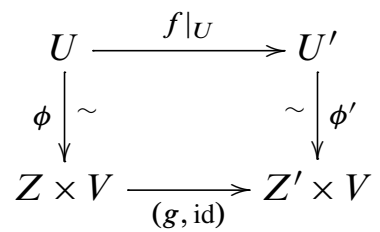


(ii) Let $(\mathfrak{X}, \mathcal{A})$ and $\left(\mathfrak{X}^{\prime}, \mathcal{A}^{\prime}\right)$ be two relative analytic spaces over $B$ and let $f: \mathfrak{X} \rightarrow \mathfrak{X}^{\prime}$ be a continuous map over $B$. We say that $f$ is a morphism when there exists a relative atlas $\widetilde{\mathcal{A}}$ equivalent to $\mathcal{A}$ such that for any relative holomorphic chart $\phi$ in $\tilde{\mathcal{A}}$, there exists a relative holomorphic chart $\phi^{\prime}$ in $\mathcal{A}^{\prime}$ such that and $\phi$ and $\phi^{\prime}$ are $f$-compatible.

(iii) Let $(\mathfrak{X}, \mathcal{A})$ and $\left(\mathfrak{X}^{\prime}, \mathcal{A}^{\prime}\right)$ be two relative analytic spaces over $B$ and $B^{\prime}$ and $f: \mathfrak{X} \longrightarrow \mathfrak{X}^{\prime}$ be a continuous map. We say that $f$ is a weak morphism if there exist a smooth orbifold map $u: B \longrightarrow B^{\prime}$ and a morphism $\tilde{f}: \mathfrak{X} \longrightarrow \mathfrak{X}^{\prime} \times{ }_{B^{\prime}} B$ such that $f$ is obtained by composing $\tilde{f}$ with the base change map from $\mathfrak{X}^{\prime} \times{ }_{B^{\prime}} B$ to $\mathfrak{X}^{\prime}$ induced by $u$.

If $f: \mathfrak{X} \longrightarrow \mathfrak{X}^{\prime}$ is a weak morphism, then the sheaf $f^{-1} \mathcal{O}_{\mathfrak{X}^{\prime}}^{\text {rel }}$ is a subsheaf of $\mathcal{O}_{\mathfrak{X}}^{\text {rel }}$. Therefore we get a pullback functor $f^{*}: \operatorname{Mod}\left(\mathcal{O}_{\mathfrak{X}^{\prime}}^{\text {rel }}\right) \longrightarrow \operatorname{Mod}\left(\mathcal{O}_{\mathfrak{X}}^{\text {rel }}\right)$ given by the formula $f^{*} \mathcal{F}=f^{-1} \mathcal{F} \otimes f^{-1} \mathcal{O}_{\mathfrak{X}^{\prime}}^{\text {rel }} \mathcal{O}_{\mathfrak{X}}^{\text {rel }}$.

To prove that many usual operations on $\operatorname{Mod}\left(\mathcal{O}_{\mathfrak{X}}^{\text {rel }}\right)$ induce operations on relatively coherent sheaves, we use a flatness lemma:

Lemma 3.5 Let $W$ be an open subset of $\mathbb{R}^{n}, G$ be a finite group of diffeomorphisms of $W$ and $Z$ be a smooth analytic set. If $V=W / G$, then $\mathcal{O}_{Z \times V}^{\text {rel }}$ is flat over $\operatorname{pr}_{1}^{-1} \mathcal{O}_{Z}$.

Proof Let $\delta: W \longrightarrow V$ be the projection and $\mathcal{M}$ be a sheaf of $\operatorname{pr}_{1}^{-1} \mathcal{O}_{Z}$-modules. Then

$$
\begin{aligned}
(\delta, \text { id })^{-1}\left(\mathcal{M} \otimes_{\mathrm{pr}_{1}^{-1} \mathcal{O}_{Z}} \mathcal{O}_{Z \times V}^{\text {rel }}\right) & =(\delta, \text { id })^{-1} \mathcal{M} \otimes_{\mathrm{pr}_{1}^{-1} \mathcal{O}_{Z}}\left(\mathcal{O}_{Z \times W}^{\text {rel }}\right)^{G} \\
& \simeq\left[(\delta, \text { id })^{-1} \mathcal{M} \otimes_{\mathrm{pr}_{1}^{-1} \mathcal{O}_{Z}} \mathcal{O}_{Z \times W}^{\text {rel }}\right]^{G} .
\end{aligned}
$$

Since the functor $\mathcal{F} \longmapsto \mathcal{F}^{G}$ from $\operatorname{Mod}_{G}\left(\mathcal{O}_{Z \times W}^{\text {rel }}\right)$ to $\operatorname{Mod}\left[\left(\mathcal{O}_{Z \times W}^{\text {rel }}\right)^{G}\right]$ is exact, it suffices to prove that $\mathcal{O}_{Z \times W}^{\text {rel }}$ is smooth over $\operatorname{pr}_{1}^{-1} \mathcal{O}_{Z}$. Let $k=\lfloor(n+1) / 2\rfloor$. Then $W \times \mathbb{R}^{k}$ can be seen as an open subset $\widetilde{W}$ in $\mathbb{C}^{(n+k) / 2}$. By [31, Theorem 2 bis] $\mathcal{O}_{Z \times \widetilde{W}}^{\text {rel }}$ is flat over $\mathcal{O}_{Z \times \widetilde{W}}$, and $\mathcal{O}_{Z \times \widetilde{W}}$ is flat over $\operatorname{pr}_{1}^{-1} \mathcal{O}_{Z}$. Therefore $\mathcal{O}_{Z \times \widetilde{W}}^{\text {rel }}$ is flat over $\operatorname{pr}_{1}^{-1} \mathcal{O}_{Z}$. If $q: Z \times \widetilde{W} \longrightarrow Z \times W$ is the projection, then $q^{-1} \mathcal{O}_{Z \times W}^{\text {rel }}{ }^{\longrightarrow}$ is a direct factor of $\mathcal{O}_{Z \times \widetilde{W}}^{\text {rel }}$ in $\operatorname{Mod}\left(\operatorname{pr}_{1}^{-1} \mathcal{O}_{Z}\right)$, so that $\mathcal{O}_{Z \times W}^{\text {rel }}$ is flat over $\operatorname{pr}_{1}^{-1} \mathcal{O}_{Z}$.

We obtain as a consequence:

Proposition $3.6 \quad$ (i) Let $(\mathfrak{X}, \mathcal{A})$ be a relative analytic space over $B$ and $\mathcal{F}, \mathcal{G}$ be relatively coherent sheaves on $\mathfrak{X}$. Then for every nonnegative integer $k$, $\mathcal{T o r}_{\mathcal{O}_{\mathfrak{X}}^{\text {rel }}}^{k}(\mathcal{F}, \mathcal{G})$ and $\mathcal{E} x t_{\mathcal{O}_{\mathfrak{X}}^{\text {rel }}}^{k}(\mathcal{F}, \mathcal{G})$ are relatively coherent on $\mathfrak{X}$. 
(ii) Let $f: \mathfrak{X} \longrightarrow \mathfrak{X}^{\prime}$ be a weak morphism between two relative analytic spaces $(\mathfrak{X}, \mathcal{A})$ and $\left(\mathfrak{X}^{\prime}, \mathcal{A}^{\prime}\right)$, and $\mathcal{G}$ be a relatively coherent sheaf on $\mathfrak{X}^{\prime}$. Then for every nonnegative integer $k, \mathcal{T}_{\text {or }} f^{-1} \mathcal{O}_{\mathfrak{X}^{\prime}}^{\text {rel }}\left(f^{-1} \mathcal{G}, \mathcal{O}_{\mathfrak{X}}^{\text {rel }}\right)$ is relatively coherent on $\mathfrak{X}$.

(iii) Let $(\mathfrak{X}, \mathcal{A})$ be a relative analytic space over $B, \mathcal{F}$ be a relatively coherent sheaf on $\mathfrak{X}$ and $u: B^{\prime} \longrightarrow B$ be a smooth map. If $\tilde{u}: \mathfrak{X} \times{ }_{B} B^{\prime} \longrightarrow \mathfrak{X}$ is the associated base change morphism, then $\mathcal{T} r_{\widetilde{u}^{-1}}^{k} \mathcal{O}_{\mathfrak{X}}^{\text {rel }}\left(\tilde{u}^{-1} \mathcal{F}, \mathcal{O}_{\mathfrak{X} \times{ }_{B} B^{\prime}}^{\text {rel }}\right)$ vanishes for every positive integer $k$.

Proof (i) Since two equivalent relative holomorphic atlases always admit a common equivalent refinement, there exists a complete relative holomorphic atlas $\tilde{\mathcal{A}}$ on $\mathfrak{X}$ equivalent to $\mathcal{A}$ such that for every relative holomorphic chart $\phi: U \stackrel{\sim}{\longrightarrow} Z \times V$ in $\widetilde{\mathcal{A}}$,

$$
\left.\mathcal{F}\right|_{U} \simeq \phi^{-1}\left(\operatorname{pr}_{1}^{-1} \overline{\mathcal{F}} \otimes_{\mathrm{pr}_{1}^{-1} \mathcal{O}_{Z}} \mathcal{O}_{Z \times V}^{\text {rel }}\right) \quad \text { and }\left.\quad \mathcal{G}\right|_{U} \simeq \phi^{-1}\left(\operatorname{pr}_{1}^{-1} \overline{\mathcal{G}} \otimes_{\mathrm{pr}_{1}^{-1} \mathcal{O}_{Z}} \mathcal{O}_{Z \times V}^{\text {rel }}\right)
$$

where $\overline{\mathcal{F}}$ and $\overline{\mathcal{G}}$ are coherent analytic sheaves on $Z$. Then

$$
\left.\left(\mathcal{F} \otimes \mathcal{O}_{\mathfrak{X}}^{\text {rel }} \mathcal{G}\right)\right|_{U} \simeq \phi^{-1}\left(\operatorname{pr}_{1}^{-1}\left(\overline{\mathcal{F}} \otimes_{\mathcal{O}_{Z}} \overline{\mathcal{G}}\right) \otimes_{\mathrm{pr}_{1}^{-1} \mathcal{O}_{Z}} \mathcal{O}_{Z \times V}^{\text {rel }}\right)
$$

and

$$
\left.\mathcal{H o m}_{\mathcal{O}_{\mathcal{X}}^{\text {rel }}}(\mathcal{F}, \mathcal{G})\right|_{U} \simeq \phi^{-1}\left(\operatorname{pr}_{1}^{-1} \mathcal{H} \operatorname{Hom}_{\mathcal{O}_{Z}}(\overline{\mathcal{F}}, \overline{\mathcal{G}}) \otimes_{\mathrm{pr}_{1}^{-1} \mathcal{O}_{Z}} \mathcal{O}_{Z \times V}^{\text {rel }}\right) \text {. }
$$

Since $\mathcal{O}_{Z \times V}^{\text {rel }}$ is flat over $\operatorname{pr}_{1}^{-1} \mathcal{O}_{Z}$, for any nonnegative integer $k$, we obtain by derivation

$$
\left.\mathcal{T}_{\mathcal{O}_{\mathcal{X}}^{\text {rel }}}^{k}(\mathcal{F}, \mathcal{G})\right|_{U} \simeq \phi^{-1}\left(\mathcal{T o r}_{\mathcal{O}_{Z}}^{k}(\overline{\mathcal{F}}, \overline{\mathcal{G}}) \otimes_{\mathrm{pr}_{1}^{-1} \mathcal{O}_{Z}} \mathcal{O}_{Z \times V}^{\text {rel }}\right)
$$

and

$$
\left.\mathcal{E} x t_{\mathcal{O}_{\mathcal{X}}^{\text {rel }}}^{k}(\mathcal{F}, \mathcal{G})\right|_{U} \simeq \phi^{-1}\left(\mathcal{E} x t_{\mathcal{O}_{Z}}^{k}(\overline{\mathcal{F}}, \overline{\mathcal{G}}) \otimes_{\mathrm{pr}_{1}^{-1} \mathcal{O}_{Z}} \mathcal{O}_{Z \times V}^{\text {rel }}\right) \text {. }
$$

(ii) We can take refinements $\tilde{\mathcal{A}}$ and $\widetilde{\mathcal{A}}^{\prime}$ of $\mathcal{A}$ and $\mathcal{A}^{\prime}$ such that for any relative holomorphic chart $\phi: U \stackrel{\sim}{\longrightarrow} Z \times V$ in $\widetilde{\mathcal{A}}$, there exists a relative holomorphic chart $\phi^{\prime}: U^{\prime} \stackrel{\sim}{\longrightarrow} Z^{\prime} \times V^{\prime}$ in $\widetilde{\mathcal{A}}^{\prime}$, a holomorphic map $g: Z \rightarrow Z^{\prime}$, a smooth map $u: V \rightarrow V^{\prime}$ and a coherent analytic sheaf $\overline{\mathcal{G}}$ on $Z^{\prime}$ such that $f(U) \subseteq U^{\prime}, \phi^{\prime} \circ f \circ \phi^{-1}=(g, u)$ and $\left.\mathcal{G}\right|_{U^{\prime}} \simeq \phi^{\prime-1}\left(\mathrm{pr}_{1}^{-1} \overline{\mathcal{G}} \otimes_{\mathrm{pr}_{1}^{-1} \mathcal{O}_{Z^{\prime}}} \mathcal{O}_{Z^{\prime} \times V^{\prime}}^{\text {rel }}\right)$. Thus

$$
\begin{aligned}
\left(f^{-1} \mathcal{G} \otimes f^{-1} \mathcal{O}_{\mathfrak{X}^{\prime}}^{\text {rel }}\right. & \left.\mathcal{O}_{\mathfrak{X}}^{\text {rel }}\right)\left.\right|_{U} \\
& \simeq \phi^{-1}\left((g, u)^{-1}\left[\operatorname{pr}_{1}^{-1} \overline{\mathcal{G}} \otimes_{\mathrm{pr}_{1}^{-1}} \mathcal{O}_{Z^{\prime}} \mathcal{O}_{Z^{\prime} \times V^{\prime}}^{\text {rel }}\right] \otimes_{(g, u)^{-1}} \mathcal{O}_{Z^{\prime} \times V^{\prime}}^{\text {rel }} \mathcal{O}_{Z \times V}^{\text {rel }}\right) \\
& \simeq \phi^{-1}\left(\operatorname{pr}_{1}^{-1}\left(g^{-1} \overline{\mathcal{G}} \otimes_{g^{-1}} \mathcal{O}_{Z^{\prime}} \mathcal{O}_{Z}\right) \otimes_{\mathrm{pr}_{1}^{-1} \mathcal{O}_{Z}} \mathcal{O}_{Z \times V}^{\text {rel }}\right),
\end{aligned}
$$

so that for any nonnegative integer $k$, Lemma 3.5 yields

$$
\mathcal{T o r}_{f^{-1}}^{k} \mathcal{O}_{\mathfrak{X}^{\prime}}^{\text {rel }}\left(f^{-1} \mathcal{G}, \mathcal{O}_{\mathfrak{X}}^{\text {rel }}\right) \simeq \phi^{-1}\left(\operatorname{pr}_{1}^{-1} \mathcal{T o r}_{g^{-1}}^{k} \mathcal{O}_{Z^{\prime}}\left(g^{-1} \overline{\mathcal{G}}, \mathcal{O}_{Z}\right) \otimes_{\mathrm{pr}_{1}^{-1} \mathcal{O}_{Z}} \mathcal{O}_{Z \times V}^{\text {rel }}\right) \text {. }
$$

(iii) We can assume that $\mathfrak{X}=Z \times B, \mathcal{F}=\operatorname{pr}_{1}^{-1} \overline{\mathcal{F}} \otimes_{\mathrm{pr}_{1}^{-1} \mathcal{O}_{Z}} \mathcal{O}_{Z \times B}^{\text {rel }}$ and $\tilde{u}=($ id, $u)$, where $Z$ is a smooth analytic space and $\overline{\mathcal{F}}$ is a coherent analytic sheaf on $Z$. Let 
$\left(z, b^{\prime}\right)$ be an element of $Z \times B^{\prime}$, put $b=u\left(b^{\prime}\right)$, and let $E_{\bullet}$ be a free resolution of $\overline{\mathcal{F}}_{z}$. By Lemma 3.5, $E_{\bullet} \otimes_{\mathcal{O}_{z}} \mathcal{O}_{z, b}^{\text {rel }}$ is a free resolution of $\mathcal{F}_{z, b}$. Therefore, the germ of $\mathcal{T o r}_{\tilde{u}^{-1} \mathcal{O}_{\mathfrak{X}}^{\text {rel }}}^{k}\left(\tilde{u}^{-1} \mathcal{F}, \mathcal{O}_{\mathfrak{X} \times{ }_{B} B^{\prime}}^{\text {rel }}\right)$ at $\left(z, b^{\prime}\right)$ is the $k$-th cohomology group of $\left(E_{\bullet} \otimes_{\mathcal{O}_{z}} \mathcal{O}_{z, b}^{\text {rel }}\right) \otimes_{\mathcal{O}_{z, b}^{\text {rel }}} \mathcal{O}_{z, b^{\prime}}^{\text {rel }}$. The latter complex being isomorphic to $E_{\bullet} \otimes_{\mathcal{O}_{z}} \mathcal{O}_{z, b^{\prime}}^{\text {rel }}$, the result is again a consequence of Lemma 3.5.

Remark 3.7 Let $\mathcal{F}$ and $\mathcal{G}$ be two relatively coherent sheaves on a relative analytic space $\mathfrak{X}$ over $B$. Then for every $b$ in $B$ and for every nonnegative integer $k$, we have $\mathcal{T o r}^{k}(\mathcal{F}, \mathcal{G})_{b}=\mathcal{T}_{o r}{ }_{\mathcal{O}_{\mathfrak{x}_{b}}}^{k}\left(\mathcal{F}_{b}, \mathcal{G}_{b}\right)$ and $\mathcal{E} x t^{k}(\mathcal{F}, \mathcal{G})_{b}=\mathcal{E}_{x} t_{\mathcal{O}_{\mathfrak{X}_{b}}}^{k}\left(\mathcal{F}_{b}, \mathcal{G}_{b}\right)$. A similar result holds for the $\mathcal{T}$ or sheaves appearing in (ii).

\subsection{Relative analytic subspaces and direct image}

The definition of a relative analytic subspace runs as follows:

Definition 3.8 Let $(\mathfrak{X}, \mathcal{A})$ be a relative analytic space over $B$ and $\mathfrak{Z}$ be a closed subset of $\mathfrak{X}$. We say that $\mathfrak{Z}$ is a relative analytic subspace (resp. smooth relative analytic subspace) of $\mathfrak{X}$ if there exists a relative holomorphic atlas $\widetilde{\mathcal{A}}$ equivalent to $\mathcal{A}$ such that for any holomorphic chart $\phi: U \stackrel{\sim}{\longrightarrow} Z \times V$ in $\widetilde{\mathcal{A}}$ with $U \cap \mathfrak{Z} \neq \varnothing$, there exists a closed (resp. closed and smooth) analytic subspace $Z^{\prime}$ of $Z$ satisfying $\phi(U \cap \mathfrak{Z})=Z^{\prime} \times V$.

For instance, the relative incidence varieties $W_{\text {rel }}^{[m, n]}$ introduced in Definition 2.21 are relative analytic subspaces of $W_{\text {rel }}^{[n]} \times_{X^{(n)} \times X^{(m-n)}} W_{\text {rel }}^{[m]}$, they are smooth if $m=n+1$. We will study other examples in Section 3.5.

Remark 3.9 If $\mathfrak{Z}$ is a smooth relative analytic subspace of a relative analytic space $(\mathfrak{X}, \mathcal{A})$, then $\mathfrak{Z}$ is also a relative analytic space: a complete relative holomorphic atlas on $\mathfrak{Z}$ is obtained by taking the restrictions to $\mathfrak{Z}$ of the charts of $\mathcal{A}$.

The forthcoming proposition is needed to associate relatively coherent sheaves with relative analytic subspaces:

Proposition 3.10 Let $n$ and $k$ be positive integers, $\Omega$ and $W$ be open subsets of $\mathbb{C}^{n}$ and $\mathbb{R}^{k}$ respectively, and $T$ be a reduced analytic subset of $\Omega$. Assume that the ideal sheaf of $T$ is globally generated by holomorphic functions $f_{1}, \ldots, f_{d}$ on $\Omega$.

- If $g: \Omega \times W \longrightarrow \mathbb{C}$ is a relatively holomorphic function on $\Omega \times W$ such that $\left.g\right|_{T \times W} \equiv 0$, then for any $\left(z_{0}, w_{0}\right)$ in $\Omega \times W$, there exist relatively holomorphic functions $\phi_{1}, \ldots, \phi_{d}$ in a neighbourhood $U_{z_{0}, w_{0}}$ of $\left(z_{0}, w_{0}\right)$ such that $g=\sum_{i=1}^{d} \phi_{i} f_{i}$ on $U_{z_{0}}, w_{0}$.

- Furthermore, if $G$ is a finite group of diffeomorphisms of $W$ fixing $w_{0}$ and if $g$ is $G$-invariant, then the functions $\phi_{i}$ can be chosen $G$-invariant too. 
Proof Let $\left(z_{0}, w_{0}\right)$ in $\Omega \times W$. For any $z$ in $\Omega$, let $\widehat{g}_{w_{0}}(z)$ be the formal Taylor expansion of the function $w \longmapsto g(z, w)$ at $w_{0}$. Writing $\hat{g}_{w_{0}}(z)=\sum_{|I|=k} \alpha_{I}(z)\left(w-w_{0}\right)^{I}$, the hypotheses made on $g$ imply that the functions $\alpha_{I}$ are holomorphic on $\Omega$ and vanish on $T$. Therefore for every multiindex $I$ of length $k$, there exist holomorphic functions $\alpha_{I 1}, \ldots, \alpha_{I d}$ in a Stein neighbourhood $U_{z_{0}}$ of $z_{0}$ such that $\alpha_{I}=\sum_{i=1}^{d} \alpha_{I i} f_{i}$ on $U_{z_{0}}$. Hence we get $\hat{g}_{w_{0}}=\sum_{i=1}^{d}\left(\sum_{|I|=k} \alpha_{I i}\left(w-w_{0}\right)^{I}\right) f_{i}$ in $\mathcal{O}\left(U_{z_{0}}\right) \llbracket w-w_{0} \rrbracket$. If $S_{i}$ (resp. $\left.\widehat{f}_{i, z_{0}}\right)$ denotes the formal expansion of $z \longmapsto \sum_{|I|=k} \alpha_{I i}(z)\left(w-w_{0}\right)^{I}$ (resp. $\left.f_{i}\right)$ at $z_{0}$ in $\mathbb{C} \llbracket z-z_{0}, w-w_{0} \rrbracket$ (resp. in $\left.\mathbb{C} \llbracket z-z_{0} \rrbracket\right)$ and if $\hat{g}_{z_{0}, w_{0}}$ denotes the formal Taylor expansion of $g$ at $\left(z_{0}, w_{0}\right)$ in $\mathbb{C} \llbracket z-z_{0}, w-w_{0} \rrbracket$, then $\hat{g}_{z_{0}, w_{0}}=\sum_{i=1}^{d} S_{i} \widehat{f}_{i, z_{0}}$. Thus for any $\left(z_{0}, w_{0}\right)$ in $\Omega \times W, \hat{g}_{z_{0}, w_{0}}$ is divisible by $\hat{f}_{1, z_{0}}, \ldots, \hat{f}_{d, z_{0}}$ in $\mathbb{C} \llbracket z-z_{0}, w-w_{0} \rrbracket$. Since the $f_{i}$ 's are analytic, it follows from [32, Theorem 1.1', page 82] that there exist $\phi_{1}, \ldots, \phi_{d}$ in $\mathcal{C}^{\infty}(\Omega)$ such that $g=\sum_{i=1}^{d} \phi_{i} f_{i}$. It remains to prove that the functions $f_{i}$ can be chosen relatively holomorphic in a neighbourhood of any point in $\Omega \times W$.

If $\left(z_{0}, w_{0}\right)$ is an element of $\Omega \times W$ and if $r$ is an integer such that $0 \leq r \leq n$, let us consider the property $\left(\mathrm{P}_{r}\right)$ :

There exist a neighbourhood $U_{z_{0}, w_{0}}$ of $\left(z_{0}, w_{0}\right)$ in $\Omega \times W$ and smooth functions $\phi_{1}, \ldots, \phi_{d}$ on $U_{z_{0}, w_{0}}$ such that:

- $g=\sum_{i=1}^{d} \phi_{i} f_{i}$ on $U_{z_{0}, w_{0}}$.

- If $r \geq 1,1 \leq i \leq d$ and $1 \leq j \leq r$, then $\partial \phi_{i} / \partial \bar{z}_{j} \equiv 0$.

We have seen that $\left(\mathrm{P}_{0}\right)$ is true. Fix $r$ such that $0 \leq r \leq n-1$, and assume that $\left(\mathrm{P}_{r}\right)$ holds. We consider a presentation $\mathcal{O}_{z_{0}}^{q} \longrightarrow \mathcal{O}_{z_{0}}^{d} \rightarrow\left(\mathcal{J}_{T}\right)_{z_{0}} \rightarrow 0$ of the ideal sheaf of $T$ at $z_{0}$, where the first map is given by a matrix $M$ in $\mathfrak{M}_{d, q}\left(\mathcal{O}_{z_{0}}\right)$ and the second one by $\left(f_{1}, \ldots, f_{d}\right)$. If $\mathcal{O}_{r}^{\text {rel }}$ denotes the sheaf of smooth functions on $\Omega \times W$ holomorphic in the first $r$ variables, then $\mathcal{O}_{r}^{\text {rel }}$ is flat over $\operatorname{pr}_{1}^{-1} \mathcal{O}_{\Omega}$ (this is proved exactly as in Lemma 3.5). Thus we get an exact sequence

$$
\left(\mathcal{O}_{r}^{\mathrm{rel}}\right)_{z_{0}, w_{0}}^{q} \stackrel{M}{\longrightarrow}\left(\mathcal{O}_{r}^{\mathrm{rel}}\right)_{z_{0}, w_{0}}^{d} \stackrel{\left(f_{1}, \ldots, f_{d}\right)}{\longrightarrow}\left(\mathcal{O}_{r}^{\mathrm{rel}}\right)_{z_{0}, w_{0}}
$$

Pick $\phi_{1} \ldots, \phi_{d}$ in $\left(\mathcal{O}_{r}^{\text {rel }}\right)_{z_{0}, w_{0}}$ such that $g=\sum_{i=1}^{d} \phi_{i} f_{i}$. Since $g$ is relatively holomorphic, $\sum_{i=1}^{d} \partial \phi_{i} / \partial \bar{z}_{r+1} f_{i} \equiv 0$. By the exactness of (3-1), there exists an element $\left(\varphi_{j}\right)_{1 \leq j \leq q}$ in $\left(\mathcal{O}_{r}^{\text {rel }}\right)_{z_{0}, w_{0}}^{q}$ such that for every $i$ with $1 \leq i \leq d, \partial \phi_{i} / \partial \bar{z}_{r+1}=$ $\sum_{j=1}^{q} M_{i j} \varphi_{j}$. Now the map $\partial / \partial \bar{z}_{r+1}:\left(\mathcal{O}_{r}^{\text {rel }}\right)_{z_{0}, w_{0}} \longrightarrow\left(\mathcal{O}_{r}^{\text {rel }}\right)_{z_{0}, w_{0}}$ is surjective, so that there are functions $\left(\gamma_{j}\right)_{1 \leq j \leq q}$ in $\left(\mathcal{O}_{r}^{\text {rel }}\right)_{z_{0}, w_{0}}$ such that for $1 \leq j \leq q, \partial \gamma_{j} / \partial \bar{z}_{r+1}=$ $\varphi_{j}$. If $\widetilde{\phi}_{i}=\phi_{i}-\sum_{j=1}^{d} M_{i j} \gamma_{j}$ then $\sum_{i=1}^{d} \widetilde{\phi}_{i} f_{i}=\sum_{i=1}^{d} \phi_{i} f_{i}=g$ in a small neighbourhood of the point $\left(z_{0}, w_{0}\right)$ and $\partial \widetilde{\phi}_{i} / \partial \bar{z}_{r+1}=\partial \phi_{i} / \partial \bar{z}_{r+1}-\sum_{i=1}^{d} M_{i j} \partial \gamma_{j} / \partial \bar{z}_{r+1}=0$, so that $\widetilde{\phi}_{i}$ is in $\left(\mathcal{O}_{r+1}^{\text {rel }}\right)_{z_{0}}, w_{0}$. This proves that $\left(\mathrm{P}_{r+1}\right)$ holds. By a finite induction, 
we obtain that $\left(\mathrm{P}_{n}\right)$ holds. This means that $\phi_{1}, \ldots, \phi_{d}$ are relatively holomorphic in a neighbourhood of $\left(z_{0}, w_{0}\right)$.

To prove the last statement, it suffices to replace for each $i$ the function $\phi_{i}$ by the $G$-invariant function $\widetilde{\phi}_{i}$ defined by $\widetilde{\phi}_{i}(z, w)=|G|^{-1} \sum_{u \in G} \phi_{i}(z, u, w)$.

Proposition 3.11 Let $\mathfrak{X}$ be a relative analytic space over $B$ and $\mathfrak{Z}$ be a relative analytic subspace of $\mathfrak{X}$. If $\mathcal{J}_{\mathfrak{Z}}^{\text {rel }}$ is the ideal sheaf of $\mathfrak{Z}$ in $\mathcal{O}_{\mathfrak{X}}^{\text {rel }}$ consisting of the relative holomorphic functions vanishing on $\mathfrak{Z}$ and if $\mathcal{O}_{\mathfrak{Z}}^{\text {rel }}=\mathcal{O}_{\mathfrak{X}}^{\text {rel }} / \stackrel{\mathcal{J}}{\mathfrak{Z}}^{\text {rel }}$ is the structure sheaf of $\mathfrak{Z}$, then $\mathcal{J}_{\mathfrak{Z}}^{\text {rel }}$ and $\mathcal{O}_{\mathfrak{Z}}^{\text {rel }}$ are relatively coherent on $\mathfrak{X}$.

Proof Since the result is local, we can assume that $\mathfrak{X}=Z \times V$ and that $\mathfrak{Z}=Z^{\prime} \times V$, where $V$ is a differentiable orbifold, $Z$ is a smooth analytic space and $Z^{\prime}$ is an analytic subset of $Z$. If $\mathcal{J}_{Z^{\prime}}$ is the ideal sheaf of $Z^{\prime}$ in $Z$, then $\mathcal{J}_{\mathfrak{Z}}^{\text {rel }}=\operatorname{pr}_{1}^{-1} \mathcal{J}_{Z^{\prime}}$. $\mathcal{O}_{\mathfrak{X}}^{\text {rel }}$ by Proposition 3.10. By Lemma 3.5, $\mathcal{O}_{\mathfrak{X}}^{\text {rel }}$ is flat over $\operatorname{pr}_{1}^{-1} \mathcal{O}_{Z}$, so the equality $\operatorname{pr}_{1}^{-1} \mathcal{J}_{Z^{\prime}}$.

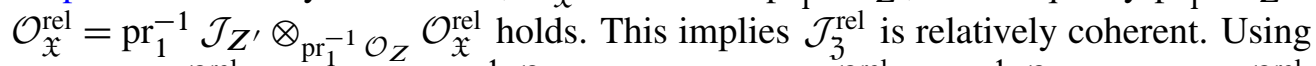
again that $\mathcal{O}_{\mathfrak{X}}^{\text {rel }}$ is flat over $\operatorname{pr}_{1}^{-1} \mathcal{O}_{Z}$, we obtain that $\mathcal{O}_{\mathfrak{Z}}^{\text {rel }}=\operatorname{pr}_{1}^{-1} \mathcal{O}_{Z^{\prime}} \otimes_{p_{1}^{-1} \mathcal{O}_{Z}} \mathcal{O}_{\mathfrak{X}}^{\text {rel }}$, and thus the sheaf $\mathcal{O}_{\mathfrak{Z}}^{\text {rel }}$ is relatively coherent too.

Let us make an important remark:

Remark 3.12 Let $Z$ be any reduced analytic set and $V$ be a differentiable orbifold. Then it is possible to define a sheaf $\mathcal{O}_{Z \times V}^{\text {rel }}$ of relative holomorphic functions on $Z \times V$ (which is a subsheaf of $C_{Z \times V}^{0}$ ) as follows: since we can argue locally, we assume that there exists an open set $U$ in some $\mathbb{C}^{n}$ such that $Z$ is a reduced analytic subset of $U$. Then we define $\mathcal{O}_{Z \times V}^{\text {rel }}$ as the structure sheaf of $Z \times V$ in $U \times V$. Using Proposition 3.11 , it is easy to prove that the definition in independent of $U$. This makes it possible to construct singular relative analytic spaces, although we will not go any further in this direction.

Following the strategy of [20, Chapter 1, Section 3], we prove:

Proposition 3.13 Let $f: \mathfrak{X} \longrightarrow \mathfrak{X}^{\prime}$ be a morphism between two relative analytic spaces $(\mathfrak{X}, \mathcal{A})$ and $\left(\mathfrak{X}^{\prime}, \mathcal{A}^{\prime}\right), \mathfrak{Z}$ be a relative analytic subspace of $\mathfrak{X}$ such that $f$ is finite on $\mathfrak{Z}$ and $\mathcal{F}$ be a relatively coherent sheaf on $\mathfrak{X}$ supported in $\mathfrak{Z}$. Then $f_{*} \mathcal{F}$ is relatively coherent on $\mathfrak{X}^{\prime}$.

Proof For any point $x^{\prime}$ in $\mathfrak{X}^{\prime}$, let $\phi^{\prime}: U^{\prime} \stackrel{\sim}{\longrightarrow} Z^{\prime} \times V$ be a relative holomorphic chart of $\mathcal{A}^{\prime}$ in a neighbourhood $U^{\prime}$ of $x^{\prime}$. Since $f$ is finite on $\mathfrak{Z}$, there exists a relative holomorphic chart $\phi: U \stackrel{\sim}{\longrightarrow} Z \times V$ in a neighbourhood of $f^{-1}\left(x^{\prime}\right) \cap \mathfrak{Z}$ such $\phi$ and $\phi^{\prime}$ are $f$-adapted. Up to a refinement of $\mathcal{A}$, we can assume that: 
- $\phi(U) \subseteq U^{\prime}$ and $\phi^{-1}\left(U^{\prime}\right) \cap \mathfrak{Z} \subseteq U$.

- $\phi^{\prime} \circ f \circ \phi^{-1}=\left(g\right.$,id), where $g: Z \longrightarrow Z^{\prime}$ is holomorphic.

- $\phi(U \cap \mathfrak{Z})=Y \times V$, where $Y$ is a reduced analytic subset of $Z$.

- The function $\left.g\right|_{Y}: Y \longrightarrow Z^{\prime}$ is finite.

- The sheaf $\left.\mathcal{F}\right|_{U}$ is isomorphic to $\phi^{-1}\left(\operatorname{pr}_{1}^{-1} \overline{\mathcal{F}} \otimes_{\mathrm{pr}_{1}^{-1}} \mathcal{O}_{Z} \mathcal{O}_{Z \times V}^{\text {rel }}\right)$, where $\overline{\mathcal{F}}$ is a coherent analytic sheaf on $Z$ supported in $Y$.

Let us prove that the natural morphism

$$
\operatorname{pr}_{1}^{-1}\left(g_{*} \overline{\mathcal{F}}\right) \otimes_{\mathrm{pr}_{1}^{-1} \mathcal{O}_{Z^{\prime}}} \mathcal{O}_{Z^{\prime} \times V}^{\text {rel }} \longrightarrow(g, \text { id })_{*}\left(\operatorname{pr}_{1}^{-1} \overline{\mathcal{F}} \otimes_{\mathrm{pr}_{1}^{-1} \mathcal{O}_{Z}} \mathcal{O}_{Z \times V}^{\text {rel }}\right)
$$

is an isomorphism. The function $g$ being finite on $Y$ and $\mathcal{O}_{Z \times V}^{\text {rel }}\left(\right.$ resp. $\mathcal{O}_{Z^{\prime} \times V}^{\text {rel }}$ ) being flat over $\mathrm{pr}_{1}^{-1} \mathcal{O}_{Z}$ (resp. $\mathrm{pr}_{1}^{-1} \mathcal{O}_{Z^{\prime}}$ ), both members of (3-2) define exact functors from $\operatorname{Coh}_{Y}(Z)$ to $\operatorname{Mod}\left(\mathcal{O}_{Z^{\prime} \times V}^{\text {rel }}\right)$. Let $\mathcal{J}_{Y}$ be the ideal sheaf of $Y$. Since $g$ is proper, we can assume, after shrinking $Z^{\prime}$ if necessary, that $\mathcal{J}_{Y}^{N} \overline{\mathcal{F}}$ vanishes for $N$ large enough. Using the exact sequences

$$
0 \longrightarrow \mathcal{J}_{Y}^{N+1} \overline{\mathcal{F}} \longrightarrow \mathcal{J}_{Y}^{N} \overline{\mathcal{F}} \longrightarrow \mathcal{J}_{Y}^{N} \overline{\mathcal{F}} / \mathcal{J}_{Y}^{N+1} \overline{\mathcal{F}} \longrightarrow 0,
$$

we see that it is sufficient to prove that (3-2) is an isomorphism when $\overline{\mathcal{F}}$ is an $\mathcal{O}_{Y}$-module. Under this assumption, for any $z^{\prime}$ in $g(Y)$, we can take local $\mathcal{O}_{Y}-$ presentations of $\overline{\mathcal{F}}$ in a neighbourhood of the finite set $g^{-1}\left(z^{\prime}\right) \cap Y$. Thus it is enough to prove that (3-2) is an isomorphism when $\overline{\mathcal{F}}=\mathcal{O}_{Y}$. If $h=\left.g\right|_{Y}$, this amounts to show that the natural morphism from $h_{*} \mathcal{O}_{Y} \otimes_{\mathrm{pr}_{1}^{-1} \mathcal{O}_{Z^{\prime}}} \mathcal{O}_{Z^{\prime} \times V}^{\text {rel }}$ to $(h, \mathrm{id})_{*} \mathcal{O}_{Y \times V}^{\text {rel }}$ is an isomorphism. Since the problem is local, we can assume that there exist two positive integers $m$ and $n$ and an open subset $\Omega$ of $\mathbb{C}^{m}$ such that $Z^{\prime}$ is open in $\mathbb{C}^{n}, Y$ is a closed analytic subset of $\Omega \times Z^{\prime}$ and $h$ is the restriction to $Y$ of the projection from $\mathbb{C}^{m} \times \mathbb{C}^{n}$ to $\mathbb{C}^{n}$.

We deal at first with the case $m=1$. If $\left(w, z^{\prime}\right)$ denotes the coordinates on $\mathbb{C} \times \mathbb{C}^{n}$, then for any $z^{\prime}$ in $h(Y),\left(h_{*} \mathcal{O}_{Y}\right)_{z^{\prime}}$ is free over $\mathcal{O}_{z^{\prime}}$ : a basis is given by the functions $1, w, \ldots, w^{d}$, where $d$ is the degree of $h$ (see [20, I, Section 2]). Since the general Weierstrass division theorem remains valid for relative holomorphic functions, for any $v$ in $V$, the module $\left((h, \mathrm{id})_{*} \mathcal{O}_{Y \times V}^{\text {rel }}\right)_{z^{\prime}, v}$ is also free over $\mathcal{O}_{z^{\prime}, v}^{\text {rel }}$ with basis $1, w, \ldots, w^{d}$. This yields the required isomorphism.

To conclude, we argue by induction on $m$. Let us write $\Omega=\Omega^{\prime \prime} \times \Omega^{\prime}$, where $\Omega^{\prime \prime}$ and $\Omega^{\prime}$ are open in $\mathbb{C}$ and $\mathbb{C}^{m-1}$ respectively, and let $p: \Omega \times Z^{\prime} \longrightarrow \Omega^{\prime} \times Z^{\prime}$ and $q: \Omega^{\prime} \times Z^{\prime} \longrightarrow Z^{\prime}$ be the natural projections. Then there exists an analytic hypersurface $S$ in $\Omega \times Z^{\prime}$ containing $Y$ such that $p$ is finite on $S$. If $\tilde{Y}=p(Y)$, then $\tilde{Y}$ is 
an analytic subset of $\Omega^{\prime} \times Z^{\prime}$ and $q$ is finite on $\tilde{Y}$. By Proposition 3.11, $\mathcal{O}_{Y \times V}^{\text {rel }}$ is,

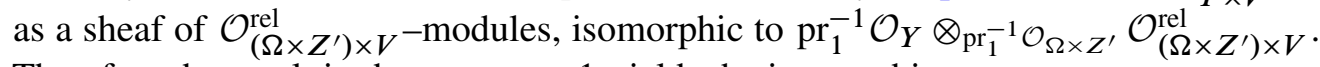
Therefore the result in the case $m=1$ yields the isomorphism

$$
p_{*} \mathcal{O}_{Y} \otimes_{\mathrm{pr}_{1}^{-1} \mathcal{O}_{\Omega^{\prime} \times Z^{\prime}}} \mathcal{O}_{\left(\Omega^{\prime} \times Z^{\prime}\right) \times V}^{\mathrm{rel}} \simeq(p, \mathrm{id})_{*} \mathcal{O}_{Y \times V}^{\mathrm{rel}} .
$$

Besides, we get another isomorphism by induction, namely

$$
q_{*}\left(p_{*} \mathcal{O}_{Y}\right) \otimes_{\mathrm{pr}_{1}^{-1} \mathcal{O}_{Z^{\prime}}} \mathcal{O}_{Z^{\prime} \times V}^{\text {rel }} \simeq(q, \mathrm{id})_{*}\left(p_{*} \mathcal{O}_{Y} \otimes_{\mathrm{pr}_{1}^{-1} \mathcal{O}_{\Omega^{\prime} \times Z^{\prime}}} \mathcal{O}_{\left(\Omega^{\prime} \times Z^{\prime}\right) \times V}^{\text {rel }}\right) .
$$

Putting these two isomorphisms together, we get the result.

\subsection{Relative analytic $K$-theory}

We are now going to introduce morphisms of relatively coherent sheaves. A natural idea would be to consider relatively coherent sheaves on a relative analytic space $(\mathfrak{X}, \mathcal{A})$ as a full subcategory of the abelian category $\operatorname{Mod}\left(\mathcal{O}_{\mathfrak{X}}^{\text {rel }}\right)$ of sheaves of $\mathcal{O}_{\mathfrak{X}}^{\text {rel }}$-modules on $\mathfrak{X}$. Unfortunately, the resulting category would be nonabelian. Indeed, if $\mathfrak{X}=Z \times B$ is trivial, if $\mathcal{F}$ is relatively coherent on $\mathfrak{X}$ and if $\chi$ is a smooth cut-off function in $B$, the multiplication by $\chi$ defines an endomorphism of $\mathcal{F}$ over $\mathcal{O}_{\mathfrak{X}}^{\text {rel }}$ whose kernel is far from being relatively coherent in general. Now, if $\mathcal{A}$ is given by the family of relative holomorphic charts $\left\{\phi_{i}: U_{i} \stackrel{\sim}{\longrightarrow} Z_{i} \times V_{i}\right\}_{i \in I}$, another natural definition is to glue together the abelian categories $\left(\operatorname{Coh}\left(Z_{i}\right)\right)_{i \in I}$ of coherent analytic sheaves on $Z_{i}$. This is what we do.

Definition 3.14 (i) A strict morphism between to relatively coherent sheaves $\mathcal{F}$ and $\mathcal{G}$ on a relative analytic space $(\mathfrak{X}, \mathcal{A})$ is a morphism $u$ in $\operatorname{Hom}_{\mathcal{O}_{\mathfrak{X}}^{\text {rel }}}(\mathcal{F}, \mathcal{G})$ satisfying the following condition: there exists a relative holomorphic atlas $\widetilde{\mathcal{A}}$ equivalent to $\mathcal{A}$ such that for every relative holomorphic chart $\phi: U \stackrel{\sim}{\longrightarrow} Z \times V$ in $\tilde{\mathcal{A}}$, there exist two coherent analytic sheaves $\overline{\mathcal{F}}$ and $\overline{\mathcal{G}}$ on $Z$ as well as a morphism $v$ in $\operatorname{Hom}_{\mathcal{O}_{Z}}(\overline{\mathcal{F}}, \overline{\mathcal{G}})$ such that $\left.\mathcal{F}\right|_{U} \simeq \phi^{-1}\left(\operatorname{pr}_{1}^{-1} \overline{\mathcal{F}} \otimes_{\mathrm{pr}_{1}^{-1} \mathcal{O}_{Z}} \mathcal{O}_{Z \times V}^{\text {rel }}\right)$, $\left.\mathcal{G}\right|_{U} \simeq \phi^{-1}\left(\operatorname{pr}_{1}^{-1} \overline{\mathcal{G}} \otimes_{\mathrm{pr}_{1}^{-1} \mathcal{O}_{Z}} \mathcal{O}_{Z \times V}^{\text {rel }}\right)$ and the following diagram commutes up to isomorphism:

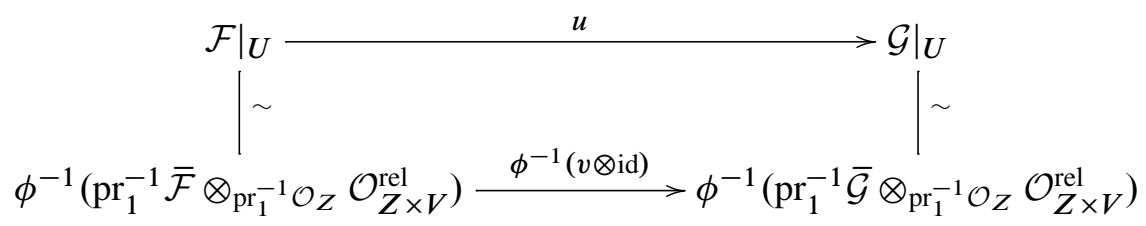

(ii) If $\mathfrak{X}$ is a relative analytic space and $\mathfrak{Z}$ is a closed subspace of $\mathfrak{X}$, we call $\mathrm{Coh}^{\text {rel }}(\mathfrak{X})$ $\left(\right.$ resp. $\left.\operatorname{Coh}_{\mathfrak{Z}}^{\text {rel }}(\mathfrak{X})\right)$ the subcategory of $\operatorname{Mod}\left(\mathcal{O}_{\mathfrak{X}}^{\text {rel }}\right)$ whose objects are relatively coherent sheaves on $\mathfrak{X}$ (resp. relatively coherent sheaves on $\mathfrak{X}$ supported in $\mathfrak{Z}$ ) and whose morphisms are strict morphisms. 
If $\mathfrak{X}$ is a relative analytic space and $\mathfrak{Z}$ is a closed subset of $\mathfrak{X}$, the categories $\operatorname{Coh}^{\text {rel }}(\mathfrak{X})$ and $\operatorname{Coh}_{\mathfrak{Z}}^{\text {rel }}(\mathfrak{X})$ are abelian subcategories of $\operatorname{Mod}\left(\mathcal{O}_{\mathfrak{X}}^{\text {rel }}\right)$ by Lemma 3.5.

In the sequel, the Grothendieck group of an abelian category $\mathcal{C}$ is denoted by $K(\mathcal{C})$.

Definition 3.15 Let $\mathfrak{X}$ be a relative analytic space and $\mathfrak{Z}$ be a closed subset of $\mathfrak{X}$. The relative analytic $K$-theory of $\mathfrak{X}$ (resp. relative analytic $K$-theory of $\mathfrak{X}$ with support in $\mathfrak{Z})$ is defined by $K^{\text {rel }}(\mathfrak{X})=K\left(\operatorname{Coh}^{\text {rel }}(\mathfrak{X})\right)\left(\operatorname{resp} . K_{\mathfrak{Z}}^{\text {rel }}(\mathfrak{X})=K\left(\operatorname{Coh}_{\mathfrak{Z}}^{\text {rel }}(\mathfrak{X})\right)\right)$.

As for coherent sheaves, we can define usual operations on relative analytic $K$-theory. These definitions rely on Proposition 3.6 and Proposition 3.13.

- (Product) If $\mathfrak{X}$ is a relative analytic space and $\mathfrak{Z}$ is a closed subset of $\mathfrak{X}$, a product from $K^{\text {rel }}(\mathfrak{X}) \otimes_{\mathbb{Z}} K^{\text {rel }}(\mathfrak{X})\left(\operatorname{resp} . K^{\text {rel }}(\mathfrak{X}) \otimes_{\mathbb{Z}} K_{\mathfrak{Z}}^{\text {rel }}(\mathfrak{X})\right)$ to $K^{\mathrm{rel}}(\mathfrak{X})$ (resp. $\left.K_{\mathfrak{Z}}^{\mathrm{rel}}(\mathfrak{X})\right)$ is defined by

$$
\mathcal{F} \cdot \mathcal{G}=\sum_{k \geq 0}(-1)^{k} \mathcal{T} r_{\mathcal{O}_{\mathfrak{X}}^{\text {rel }}}^{k}(\mathcal{F}, \mathcal{G})
$$

- (Dual morphism) Let $\mathfrak{X}$ be a relative analytic space and $\mathfrak{Z}$ be a closed subset of $\mathfrak{X}$. The dual morphism $\mathcal{F} \longmapsto \mathcal{F}^{\vee}$ from $K^{\text {rel }}(\mathfrak{X})$ to $K^{\text {rel }}(\mathfrak{X})$ (resp. from $K_{\mathfrak{Z}}^{\text {rel }}(\mathfrak{X})$ to $K_{\mathfrak{Z}}^{\text {rel }}(\mathfrak{X})$ ) is given by

$$
\mathcal{F}^{\vee}=\sum_{k \geq 0}(-1)^{k} \mathcal{E} x t_{\mathcal{O}_{\mathfrak{X}}^{\text {rel }}}^{k}\left(\mathcal{F}, \mathcal{O}_{\mathfrak{X}}^{\text {rel }}\right)
$$

- (Pullback morphism) Let $f: \mathfrak{X} \longrightarrow \mathfrak{X}^{\prime}$ be a weak morphism between relative analytic spaces and $\mathfrak{Z}^{\prime}$ be a closed subset of $\mathfrak{X}^{\prime}$. The pullback morphism (resp. the pullback morphism with support) $f^{!}: K^{\mathrm{rel}}\left(\mathfrak{X}^{\prime}\right) \rightarrow K^{\mathrm{rel}}(\mathfrak{X})$ (resp. $\left.f^{!}: K_{\mathfrak{Z}^{\prime}}^{\text {rel }}\left(\mathfrak{X}^{\prime}\right) \rightarrow K_{f^{-1}\left(\mathfrak{Z}^{\prime}\right)}^{\text {rel }}\left(\mathfrak{X}^{\prime}\right)\right)$ is defined by

$$
f^{!} \mathcal{G}=\sum_{k \geq 0}(-1)^{k} \mathcal{T o r}_{f^{-1} \mathcal{O}_{\mathfrak{X}^{\prime}}^{\text {rel }}}\left(f^{-1} \mathcal{G}, \mathcal{O}_{\mathfrak{X}}^{\text {rel }}\right) \text {. }
$$

- (Gysin morphism) Let $f: \mathfrak{X} \longrightarrow \mathfrak{X}^{\prime}$ be a morphism between two relative analytic spaces and $\mathfrak{Z}$ be a relative analytic subset of $\mathfrak{X}$ such that $f$ is finite on Z. The Gysin morphism $f_{*}$ from $K_{\mathfrak{Z}}^{\mathrm{rel}}(\mathfrak{X})$ to $K^{\mathrm{rel}}\left(\mathfrak{X}^{\prime}\right)$ is induced by the exact functor $f_{*}: \operatorname{Coh}_{\mathfrak{Z}}^{\text {rel }}(\mathfrak{X}) \longrightarrow \operatorname{Coh}\left(\mathfrak{X}^{\prime}\right)$.

We now list all the properties we need concerning the operations introduced above. 
Proposition $3.16 \quad$ (i) (Product structure) If $\mathfrak{X}$ is a relative analytic space and if $\mathfrak{Z}$ is a closed subspace of $\mathfrak{X}$, then $K^{\mathrm{rel}}(\mathfrak{X})$ is a unitary ring and $K_{\mathfrak{Z}}^{\mathrm{rel}}(\mathfrak{X})$ is a module over $K^{\mathrm{rel}}(\mathfrak{X})$. Besides, if $\mathcal{F}$ and $\mathcal{G}$ are relatively coherent sheaves on $\mathfrak{X}$ and if $\mathcal{G}$ is supported in $\mathfrak{Z}$, then

$$
\mathcal{F}^{\vee} \cdot \mathcal{G}=\sum_{i \geq 0}(-1)^{i} \mathcal{E} x t_{\mathcal{O}_{\mathfrak{X}}^{\text {rel }}}^{i}(\mathcal{F}, \mathcal{G}) \quad \text { in } \quad K_{\mathfrak{Z}}^{\text {rel }}(\mathfrak{X})
$$

(ii) (Functoriality) The pullback morphism (resp. Gysin morphism) in relative $K$-theory is contravariant (resp. covariant) with respect to weak morphisms (resp. with respect to morphisms). Besides, the pullback and the dual morphism commute.

(iii) (Projection formula) Let $f: \mathfrak{X} \longrightarrow \mathfrak{X}^{\prime}$ be a morphism between two relative analytic spaces and $\mathfrak{Z}$ be a relative analytic subspace of $\mathfrak{X}$ such that $f$ is finite on $\mathfrak{Z}$. If $\mathcal{F}$ is a relatively coherent sheaf on $\mathfrak{X}$ supported in $\mathfrak{Z}$ and $\mathcal{G}$ is a relatively coherent sheaf on $\mathfrak{X}^{\prime}$, then $f_{*}\left(\mathcal{F} . f^{!} \mathcal{G}\right)=f_{*} \mathcal{F} \cdot \mathcal{G}$ in $K^{\mathrm{rel}}\left(\mathfrak{X}^{\prime}\right)$.

(iv) (Base change I) Let $f: \mathfrak{X} \longrightarrow \mathfrak{X}^{\prime}$ be a morphism between two relative analytic spaces over $B, \Delta$ be a relative analytic space over $B$ and $\mathfrak{Z}^{\prime}$ be a relative analytic subspace of $\mathfrak{X}^{\prime} \times_{B} \Delta$ such that the projection $q$ : $\mathfrak{X}^{\prime} \times_{B} \Delta \longrightarrow \mathfrak{X}^{\prime}$ is finite on $\mathfrak{Z}^{\prime}$. If $f_{\Delta}=f \times_{B} \mathrm{id}_{\Delta}$, we consider the cartesian diagram:

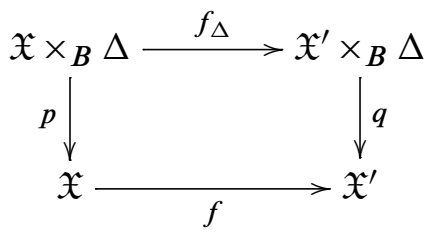

If $\mathfrak{Z}=f_{\Delta}^{-1}\left(\mathfrak{Z}^{\prime}\right)$, then $\mathfrak{Z}$ is a relative analytic subspace of $\mathfrak{X} \times{ }_{B} \Delta$ and $p$ is finite on $\mathfrak{Z}$. Besides, the pullback and Gysin morphisms

$$
\begin{aligned}
& p_{*}: \quad K_{\mathfrak{Z}}^{\mathrm{rel}}\left(\mathfrak{X} \times_{B} \Delta\right) \longrightarrow K^{\mathrm{rel}}(\mathfrak{X}), \quad q_{*}: \quad K_{\mathfrak{Z}^{\prime}}^{\mathrm{rel}}\left(\mathfrak{X}^{\prime} \times_{B} \Delta\right) \longrightarrow K^{\mathrm{rel}}\left(\mathfrak{X}^{\prime}\right), \\
& f^{!}: K^{\mathrm{rel}}\left(\mathfrak{X}^{\prime}\right) \longrightarrow K^{\mathrm{rel}}(\mathfrak{X}) \quad f_{\Delta}^{!}: K_{\mathfrak{Z}^{\prime}}^{\mathrm{rel}}\left(\mathfrak{X}^{\prime} \times{ }_{B} \Delta\right) \longrightarrow K_{\mathfrak{Z}}^{\mathrm{rel}}\left(\mathfrak{X} \times{ }_{B} \Delta\right)
\end{aligned}
$$

are related through the formula $f^{!} q_{*}=p_{*} f_{\Delta}^{!}$.

(v) (Base change II) Let $\Delta$ be a relative analytic space over $B, f: \mathfrak{X} \longrightarrow \mathfrak{X}^{\prime}$ be a morphism between two relative analytic spaces over $B$ and assume that $\mathfrak{X}^{\prime}$ is a smooth relative analytic subspace of $\Delta$. If $f_{\Delta}=f \times_{B} \mathrm{id}_{\Delta}$, consider the 
cartesian diagram

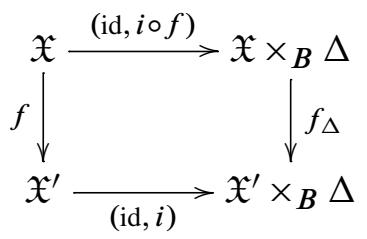

Then the pullback and Gysin morphisms

$(\mathrm{id}, i)_{*}: K^{\mathrm{rel}}\left(\mathfrak{X}^{\prime}\right) \rightarrow K_{\mathfrak{X}^{\prime}}^{\mathrm{rel}}\left(\mathfrak{X}^{\prime} \times_{B} \Delta\right),(\mathrm{id}, i \circ f)_{*}: K^{\mathrm{rel}}(\mathfrak{X}) \rightarrow K_{\mathfrak{X}}^{\mathrm{rel}}\left(\mathfrak{X} \times_{B} \Delta\right)$,

$f^{!}: K^{\mathrm{rel}}\left(\mathfrak{X}^{\prime}\right) \rightarrow K^{\mathrm{rel}}(\mathfrak{X}), \quad f_{\dot{\Delta}}^{!}: K_{\mathfrak{X}^{\prime}}^{\mathrm{rel}}\left(\mathfrak{X}^{\prime} \times_{B} \Delta\right) \rightarrow K_{\mathfrak{X}}^{\mathrm{rel}}\left(\mathfrak{X} \times{ }_{B} \Delta\right)$

are related through the formula $f_{\dot{\Delta}}^{!}(\mathrm{id}, i)_{*}=(\mathrm{id}, i \circ f)_{*} f^{!}$.

(vi) (Base change III) Let $(\mathfrak{X}, \mathcal{A})$ be a relative analytic space over $B, \mathfrak{Z}$ be a closed subset of $\mathfrak{X}, u: B \longrightarrow B^{\prime}$ be a smooth map and $\tilde{u}: \mathfrak{X} \times{ }_{B} B^{\prime} \longrightarrow \mathfrak{X}$ be the associated base change morphism. Then the pullback functor $\tilde{u}^{*}$ from $\operatorname{Coh}^{\text {rel }}(\mathfrak{X})$ to $\operatorname{Coh}^{\text {rel }}\left(\mathfrak{X} \times_{B} B^{\prime}\right)$ (resp. from $\operatorname{Coh}_{\mathfrak{Z}}^{\text {rel }}(\mathfrak{X})$ to $\operatorname{Coh}_{\widetilde{u}^{-1}(\mathfrak{Z})}^{\text {rel }}\left(\mathfrak{X} \times_{B} B^{\prime}\right)$ ) is exact, and $\tilde{u}^{!}=\tilde{u}^{*}$.

Proof (i) If $\mathcal{F}, \mathcal{G}$ and $\mathcal{H}$ are relatively coherent sheaves on $\mathfrak{X}$, there is a spectral sequence (canonical from $E_{2}$ ) such that

$$
E_{2}^{p, q}=\mathcal{T}_{o} r_{\mathcal{O}}^{p}\left(\mathcal{T}_{\mathfrak{X}}^{\text {rel }} r_{\mathfrak{X}}^{q}(\mathcal{F}, \mathcal{G}), \mathcal{H}\right), \quad E_{\infty}^{p, q}=\operatorname{Gr}_{p} \mathcal{T}_{\mathcal{O}^{\text {rel }}}^{p+q}{ }_{\mathcal{O}_{\mathfrak{X}}^{\text {rel }}}^{p}(\mathcal{F}, \mathcal{G}, \mathcal{H})
$$

and $E_{2}^{p, q}$ vanishes on each component of $\mathfrak{X}$ except for finitely many couples $(p, q)$. Furthermore, by Proposition 3.6 (i), the sheaves $E_{2}^{p, q}$ are relatively coherent on $\mathfrak{X}$ and the morphisms $d_{2}^{p, q}$ are strict. Thus, for all $r \geq 2$, the sheaves $E_{r}^{p, q}$ are relatively coherent and the morphisms $d_{r}^{p, q}$ are strict, so that

$$
\sum_{p, q \geq 0}(-1)^{p+q} E_{2}^{p, q}=\sum_{n \geq 0}(-1)^{n} \mathcal{T}_{\operatorname{or}_{\mathcal{X}}^{\text {rel }}}^{n}(\mathcal{F}, \mathcal{G}, \mathcal{H})
$$

in $K^{\text {rel }}(\mathfrak{X})$. This yields the associativity of the product. The proofs of the remaining properties in (i) and of (ii) are essentially similar, using spectral sequences associated with the composition of two functors.

The proofs of (iii), (iv) and (v) are performed in the same way. We detail the proof of (iv).

(iv) For $x$ in $\mathfrak{X}$, we take two relative holomorphic charts $\phi: U \stackrel{\sim}{\longrightarrow} Z \times V$ and $\phi^{\prime}: U^{\prime} \stackrel{\sim}{\longrightarrow} Z^{\prime} \times V$ in neighbourhoods of $x$ and $f(x)$ such that $\phi$ and $\phi^{\prime}$ are $f-$ compatible. Let us write $\phi^{\prime} \circ f \circ \phi^{-1}=(g$, id $)$, where $g: Z \longrightarrow Z^{\prime}$ is holomorphic. If $\delta_{1}, \ldots, \delta_{N}$ are elements of $\Delta$ such that $q^{-1}(f(x)) \cap \mathfrak{Z}=\bigcup_{i=1}^{N}\left(f(x), \delta_{i}\right)$, we 
choose a relative holomorphic chart $\psi: U_{\delta_{1}, \ldots, \delta_{N}} \stackrel{\sim}{\longrightarrow} Y \times V$ in a neighbourhood of the $\delta_{i}$ 's. The local form of the diagram of (iv) is then:

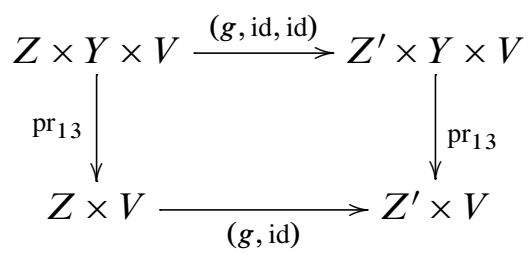

Furthermore, we can assume that $\left(\phi^{\prime} \times_{V} \psi\right)\left[\left(U^{\prime} \times U_{\delta_{1}, \ldots, \delta_{N}}\right) \cap \mathfrak{X}\right]=\overline{\mathfrak{Z}} \times V$, where $\overline{\mathfrak{Z}}$ is an analytic subset of $Z^{\prime} \times Y$ and $\mathrm{pr}_{1}$ is finite on $\overline{\mathfrak{Z}}$. Then for any coherent analytic sheaf $\overline{\mathcal{G}}$ on $Z^{\prime} \times Y$ supported in $\overline{\mathfrak{Z}}$, we use Proposition 3.13 and we get

$$
\begin{aligned}
& (g, \mathrm{id})^{*}\left[\operatorname{pr}_{13 *}\left(\operatorname{pr}_{12}^{-1} \overline{\mathcal{G}} \otimes_{\mathrm{pr}_{12}^{-1} \mathcal{O}_{Z^{\prime} \times Y}} \mathcal{O}_{Z^{\prime} \times Y \times V}^{\text {rel }}\right)\right] \\
& \simeq(g, \mathrm{id})^{*}\left(\mathrm{pr}_{1 *} \overline{\mathcal{G}} \otimes_{\mathrm{pr}_{1}^{-1}}^{-1} \mathcal{O}_{Z^{\prime}} \mathcal{O}_{Z^{\prime} \times V}^{\mathrm{rel}}\right) \\
& \simeq g^{*}\left(\operatorname{pr}_{1 *} \overline{\mathcal{G}}\right) \otimes_{\mathrm{pr}_{1}^{-1} \mathcal{O}_{Z}} \mathcal{O}_{Z \times V}^{\text {rel }} \\
& \simeq \operatorname{pr}_{1 *}\left[(g, \mathrm{id})^{*} \overline{\mathcal{G}}\right] \otimes \operatorname{pr}_{1}^{-1} \mathcal{O}_{Z} \mathcal{O}_{Z \times V}^{\mathrm{rel}} \\
& \simeq \operatorname{pr}_{13 *}\left[\operatorname{pr}_{12}^{-1}(g, \mathrm{id})^{*} \overline{\mathcal{G}} \otimes_{\mathrm{pr}_{12}^{-1}} \mathcal{O}_{Z \times Y} \mathcal{O}_{Z \times Y \times V}^{\mathrm{rel}}\right] \\
& \simeq \operatorname{pr}_{13 *}\left[(g, \mathrm{id}, \mathrm{id})^{*}\left(\operatorname{pr}_{12}^{-1} \overline{\mathcal{G}} \otimes_{\operatorname{pr}_{12}^{-1} \mathcal{O}_{Z^{\prime} \times Y}} \mathcal{O}_{Z^{\prime} \times Y \times V}^{\text {rel }}\right)\right] .
\end{aligned}
$$

Taking the derivative with respect to $\overline{\mathcal{G}}$ and using Lemma 3.5, we obtain the result.

(vi) This is an immediate consequence of Proposition 3.6 (iii).

\subsection{Topological $K$-theory for relatively coherent sheaves}

In Section 3.3, we have constructed a theory for relative coherent sheaves as well as associated operations. It remains to obtain cohomological information about these objects. To do so, we construct global resolutions by complex vector bundles for relatively coherent sheaves. We start with a general result:

Proposition 3.17 Let $Y$ be a differentiable orbifold.

(i) Locally free sheaves of $\mathcal{C}_{Y}^{\infty}$-modules are projective elements in the category $\operatorname{Mod}\left(\mathcal{C}_{Y}^{\infty}\right)$. In particular, if $0 \longrightarrow \mathcal{F} \longrightarrow \mathcal{G} \longrightarrow \mathcal{H} \longrightarrow 0$ is an exact sequence of sheaves of $\mathcal{C}_{Y}^{\infty}$-modules on $Y$ and if $\mathcal{H}$ is locally free, then this sequence globally splits.

(ii) If $\mathcal{H}$ is sheaf of $\mathcal{C}_{Y}^{\infty}$-modules admitting a finite free resolution in a neighbourhood of any point of $Y$, then $\mathcal{H}$ admits a finite locally free resolution in a neighbourhood of any compact subset of $Y$. 
(iii) Two finite locally free resolutions of a sheaf of $\mathcal{C}_{Y}^{\infty}$-modules are subresolutions of a third one.

(iv) Let $0 \longrightarrow \mathcal{F} \longrightarrow \mathcal{G} \longrightarrow \mathcal{H} \longrightarrow 0$ be an exact sequence of sheaves of $\mathcal{C}_{Y}^{\infty}$-modules such that $\mathcal{G}$ and $\mathcal{H}$ admit finite locally free resolutions on $Y$. Then $\mathcal{F}$ admits a finite locally free resolution on $Y$ and we can find three such resolutions $F_{\bullet}, G_{\bullet}$ and $H_{\bullet}$ of $\mathcal{F}, \mathcal{G}$ and $\mathcal{H}$ related by an exact sequence $0 \longrightarrow F_{\bullet} \longrightarrow G_{\bullet} \longrightarrow H_{\bullet} \longrightarrow 0$.

Proof (i) Let $\mathcal{P}, \mathcal{Q}, \mathcal{H}$ be sheaves of $\mathcal{C}_{Y}^{\infty}$-modules such that $\mathcal{Q}$ is a quotient of $\mathcal{P}$ and $\mathcal{H}$ is locally free, and let $\pi$ be in $\operatorname{Hom}_{\mathcal{C}_{Y}^{\infty}}(\mathcal{H}, \mathcal{Q})$. It is possible to lift $\pi$ locally to a morphism from $\mathcal{H}$ to $\mathcal{P}$, and then globally using a partition of unity on $Y$.

(ii) Let $K$ be a compact subset of $Y$. We choose a finite covering $\left(U_{i}\right)_{1 \leq i \leq d}$ of $K$ and open sets $\left(V_{i}\right)_{1 \leq i \leq d}$ such that for $1 \leq i \leq d, U_{i}$ is relatively compact in $V_{i}$ and $\mathcal{H}$ admits a finite free resolution on $V_{i}$. For each $i$, we multiply this resolution by a smooth cut-off function equal to 1 on $U_{i}$ and supported in $V_{i}$. We obtain in this way a complex of sheaves

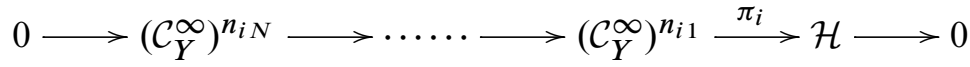

on $Y$, which is exact in $U_{i}$. If $E=\bigoplus_{i=1}^{d}\left(\mathcal{C}_{Y}^{\infty}\right)^{n_{i 1}}$ and if $\pi=\bigoplus_{i=1}^{d} \pi_{i}: E \longrightarrow \mathcal{H}$ is the sum of the $\pi_{i}$ 's, then the morphism $\pi$ is surjective in a neighbourhood of $K$. For $1 \leq i \leq d$, let $\mathcal{N}_{i}$ and $\mathcal{N}$ denote the kernels of $\pi_{i}$ and $\pi$ respectively. We have an exact sequence

$$
\left.\left.0 \longrightarrow \mathcal{N}_{i}\right|_{U_{i}} \longrightarrow \mathcal{N}\right|_{U_{i}} \longrightarrow \bigoplus_{j \neq i}\left(\mathcal{C}_{U_{i}}^{\infty}\right)^{n_{j 1}} \longrightarrow 0 .
$$

By (i), $\left.\mathcal{N}\right|_{U_{i}}$ is isomorphic to $\left.\mathcal{N}_{i}\right|_{U_{i}} \oplus\left(\mathcal{C}_{U_{i}}^{\infty}\right)^{\sum_{j \neq i} n_{j 1}}$. Furthermore $\left.\mathcal{N}_{i}\right|_{U_{i}}$ admits a finite free resolution of length $N-1$. Thus $\mathcal{N}$ admits a finite free resolution of length at most $N-1$ in a neighbourhood of every point in $K$ and we can start the argument again. After at most $N$ steps, we obtain a locally free kernel.

(iii) Let $\mathcal{H}$ be a sheaf of $\mathcal{C}_{Y}^{\infty}$-modules and $\left(E_{i}\right)_{0 \leq i \leq N}$ and $\left(F_{i}\right)_{0 \leq i \leq N}$ be two finite locally free resolutions of $\mathcal{H}$ on $Y$. Let us construct by induction a finite locally free resolution $G_{\bullet}$ of $\mathcal{H}$ on $Y$ such that $E_{\bullet}$ and $F_{\bullet}$ are subresolutions of $G_{\bullet}$. We put $G_{0}=E_{0} \oplus F_{0}$, the map from $G_{0}$ to $\mathcal{H}$ being obtained by adding the two maps from $E_{0}$ and from $F_{0}$ to $\mathcal{H}$. If $k$ is a positive integer smaller than or equal to $N-1$, assume that we have constructed $\left(G_{i}\right)_{0 \leq i \leq k}$ as well as injections $E_{\bullet} \longrightarrow G_{\bullet}$ and 
$F_{\bullet} \longrightarrow G_{\bullet}$ in degrees at most $k$. Then in the two diagrams
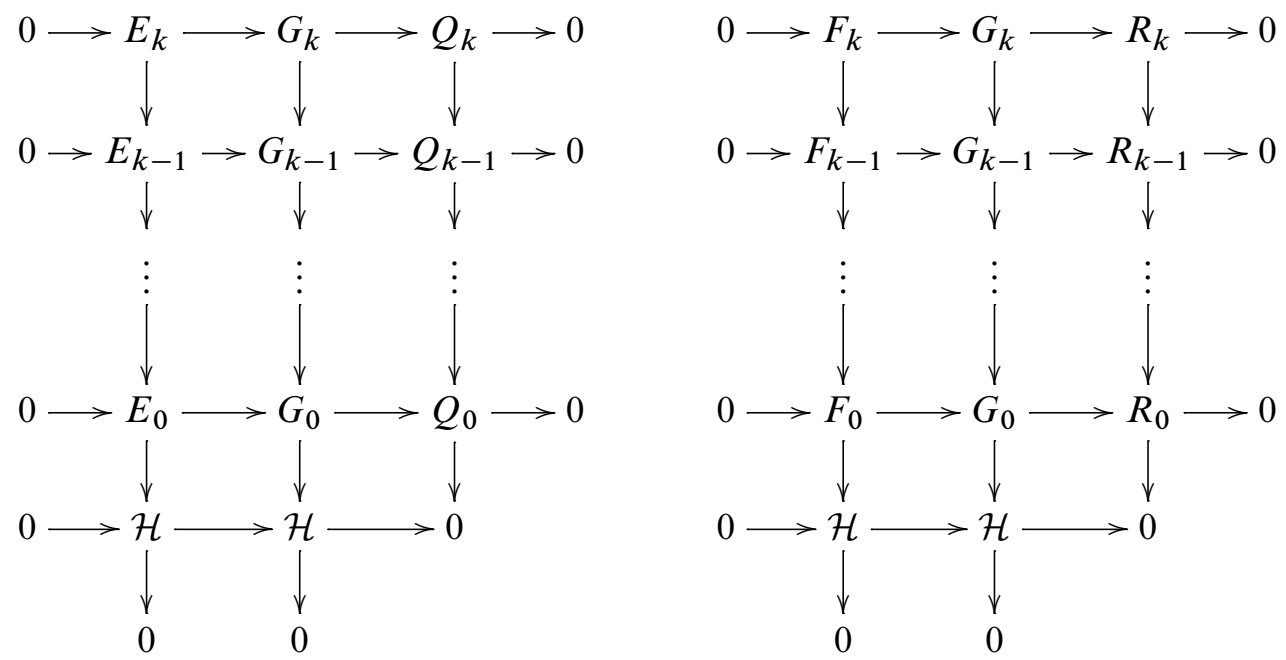

all the lines and the columns are exact and $Q_{0}, \ldots, Q_{k}, R_{0}, \ldots, R_{k}$ are locally free by induction. Let $N_{k}=\operatorname{ker}\left(E_{k} \rightarrow E_{k-1}\right), N_{k}^{\prime}=\operatorname{ker}\left(F_{k} \rightarrow F_{k-1}\right), N_{k}^{\prime \prime}=\operatorname{ker}\left(G_{k} \rightarrow G_{k-1}\right)$, $\widetilde{Q}_{k}=\operatorname{ker}\left(Q_{k} \rightarrow Q_{k-1}\right)$ and $\widetilde{R}_{k}=\operatorname{ker}\left(R_{k} \rightarrow R_{k-1}\right)$. By breaking the exact sequences of the two last columns into short exact sequences, we obtain that $\widetilde{Q}_{k}$ and $\widetilde{R}_{k}$ are locally free. The two sequences

$$
0 \longrightarrow N_{k} \longrightarrow N_{k}^{\prime \prime} \longrightarrow \widetilde{Q}_{k} \longrightarrow 0 \text { and } 0 \longrightarrow N_{k}^{\prime} \longrightarrow N_{k}^{\prime \prime} \longrightarrow \widetilde{R}_{k} \longrightarrow 0
$$

are exact. By (i), $N_{k}^{\prime \prime} \simeq N_{k} \oplus \widetilde{Q}_{k} \simeq N_{k}^{\prime} \oplus \widetilde{R}_{k}$, and we can define $G_{k+1}$ by the formula $G_{k+1}=\left(E_{k+1} \oplus \widetilde{Q}_{k}\right) \oplus\left(F_{k+1} \oplus \widetilde{R}_{k}\right)$.

If $k=N$, we end the resolution $G_{\bullet}$ by putting $G_{N+1}=N_{N}^{\prime \prime}$.

(iv) If $\left(\widetilde{G}_{i}\right)_{0 \leq i \leq N}$ and $\left(H_{i}\right)_{0 \leq i \leq N}$ are locally free resolutions of $\mathcal{G}$ and $\mathcal{H}$, let us construct by induction locally free resolutions $F_{\bullet}$ and $G_{\bullet}$ of $\mathcal{F}$ and $\mathcal{G}$ such that $\widetilde{G}_{\bullet}$ is a quotient of $G_{\bullet}$, together with an exact sequence $0 \longrightarrow F_{\bullet} \longrightarrow G_{\bullet} \longrightarrow H_{\bullet} \longrightarrow 0$.

Since $H_{0}$ (resp. $\widetilde{G}_{0}$ ) is a projective object by (i), we can lift the map from $H_{0}$ to $\mathcal{H}$ (resp. from $\widetilde{G}_{0}$ to $\mathcal{H}$ ) to a map $\pi$ (resp. $\tilde{\pi}$ ) from $H_{0}$ to $\mathcal{G}$ (resp. from $\widetilde{G}_{0}$ to $H_{0}$ ). If $G_{0}=H_{0} \oplus \widetilde{G}_{0}$, there is a natural surjective map from $G_{0}$ to $\mathcal{G}$ obtained by adding $\pi$ and the map from $\widetilde{G}_{0}$ to $\mathcal{G}$. Besides, (id, $\tilde{\pi}$ ): $G_{0} \longrightarrow H_{0}$ is surjective, we denote its kernel by $F_{0}$.

If $k$ is a positive integer smaller than or equal to $N$, assume that we have constructed $\left(F_{i}\right)_{0 \leq i \leq k}$ and $\left(G_{i}\right)_{0 \leq i \leq k}$, an exact sequence $0 \longrightarrow F_{\bullet} \longrightarrow G_{\bullet} \longrightarrow H_{\bullet} \longrightarrow 0$ and a surjective morphism from $G_{\bullet}$ to $\widetilde{G}_{\bullet}$ in degrees at most $k$. Let $N_{k}=\operatorname{ker}\left(F_{k} \rightarrow F_{k-1}\right)$, 
$N_{k}^{\prime}=\operatorname{ker}\left(G_{k} \rightarrow G_{k-1}\right), \quad N_{k}^{\prime \prime}=\operatorname{ker}\left(H_{k} \rightarrow H_{k-1}\right)$ and $\tilde{N}_{k}=\operatorname{ker}\left(\widetilde{G}_{k} \rightarrow \widetilde{G}_{k-1}\right)$. We have an exact sequence $0 \longrightarrow N_{k} \longrightarrow N_{k}^{\prime} \longrightarrow N_{k}^{\prime \prime} \longrightarrow 0$. If $R_{\bullet}=\operatorname{ker}\left(G_{\bullet} \rightarrow \widetilde{G}_{\bullet}\right)$ and $S_{k}=\operatorname{ker}\left(R_{k} \rightarrow R_{k-1}\right)$ the sequence $0 \longrightarrow S_{k} \longrightarrow N_{k}^{\prime} \longrightarrow \tilde{N}_{k} \longrightarrow 0$ is exact, so that $N_{k}^{\prime} \simeq S_{k} \oplus \tilde{N}_{k}$ by (i). As above, we lift the map from $H_{k+1}$ to $N_{k}^{\prime \prime}$ (resp. from $S_{k} \oplus \widetilde{G}_{k+1}$ to $N_{k}^{\prime \prime}$ ) to a map $\pi_{k}$ (resp. $\tilde{\pi}_{k}$ ) from $H_{k+1}$ to $N_{k}^{\prime}$ (resp. from $S_{k} \oplus \widetilde{G}_{k}$ to $H_{k+1}$ ). If $G_{k+1}=H_{k+1} \oplus S_{k} \oplus \widetilde{G}_{k+1}$, there is a natural surjection from $G_{k+1}$ to $N_{k}^{\prime}$ obtained by adding $\pi_{k}$ and the map from $S_{k} \oplus \widetilde{G}_{k+1}$ to $N_{k}^{\prime}$. Then we define $F_{k+1}$ by $F_{k+1}=\operatorname{ker}\left(\tilde{\pi}_{k}\right.$, id $)$.

If $k=N, F_{N+1}=N_{N+1}$ and $G_{N+1}=N_{N+1}^{\prime}$, so that $F_{\bullet}$ and $G_{\bullet}$ are locally free resolutions of $\mathcal{F}$ and $\mathcal{G}$.

We apply now this result in our context. If $(\mathfrak{X}, \mathcal{A})$ is a relative analytic space, then $\mathfrak{X}$ is also a differentiable orbifold, and $\mathcal{O}_{\mathfrak{X}}^{\text {rel }}$ is a subsheaf of $\mathcal{C}_{\mathfrak{X}}^{\infty}$. Therefore, we can associate with every relatively coherent analytic sheaf $\mathcal{F}$ on $\mathfrak{X}$ the sheaf $\mathcal{F}^{\infty}$ defined by $\mathcal{F}^{\infty}=\mathcal{F} \otimes_{\mathcal{O}_{\mathfrak{X}}^{\text {rel }}} \mathcal{C}_{\mathfrak{X}}^{\infty}$, which is a sheaf of $\mathcal{C}_{\mathfrak{X}}^{\infty}$-modules. This sheaf admits a finite free resolution in a neighbourhood of any point of $\mathfrak{X}$, thanks to the lemma:

Lemma 3.18 Let $U$ be an open subset of $\mathbb{R}^{n}, G$ be a finite group of diffeomorphisms of $U$ and $Z$ be a smooth analytic set. If $W=U / G$, then $\mathcal{C}_{Z \times W}^{\infty}$ is flat over $\operatorname{pr}_{1}^{-1} \mathcal{O}_{Z}$.

Proof As in Lemma 3.5, it suffices to prove that $\mathcal{O}_{Z \times U}^{\text {rel }}$ is flat over $\operatorname{pr}_{1}^{-1} \mathcal{O}_{Z}$. If $Y$ is a real-analytic manifold, let $\mathcal{C}_{Y}^{\omega}$ be the sheaf of real-analytic functions on $Y$. Then, $\mathcal{C}_{Z}^{\omega}$ is flat over $\mathcal{O}_{Z}, \mathcal{C}_{Z \times U}^{\omega}$ is flat over $\operatorname{pr}_{1}^{-1} \mathcal{C}_{Z}^{\omega}$ and $\mathcal{C}_{Z \times U}^{\infty}$ is flat over $\mathcal{C}_{Z \times U}^{\omega}$ by [31, Theorem 2].

The Grothendieck group of the category of complex topological vector bundles on a topological space $Y$ will be called $K(Y)$. Besides, the class in $K(Y)$ of a complex vector bundle $E$ on $Y$ will be denoted by $[E]$. Then Proposition 3.17 and Lemma 3.18 yield:

Proposition 3.19 If $(\mathfrak{X}, \mathcal{A})$ is a relative analytic space and if $\mathcal{F}$ is a relatively coherent sheaf on $\mathfrak{X}$, then the sheaf $\mathcal{F}^{\infty}$ admits a finite locally free resolution in a neighbourhood of any compact subset of $\mathfrak{X}$. Besides, if $\mathfrak{U}$ is a relatively compact open subset of $\mathfrak{X}$ and $\left(E_{i}\right)_{0 \leq i \leq N}$ is a locally free resolution of $\mathcal{F}^{\infty}$ on $\mathfrak{U}$, then the element $\sum_{i=0}^{N}(-1)^{i}\left[E_{i}\right]$ of $K^{\mathrm{rel}}(\mathfrak{U})$ is independent of $E_{\bullet}$ and depends only on the class of $\mathcal{F}$ in $K^{\mathrm{rel}}(\mathfrak{X})$.

In conclusion, we can associate with each relatively coherent sheaf $\mathcal{F}$ on $\mathfrak{X}$ a topological class $\left[\mathcal{F}^{\infty}\right]$ in $\lim _{\mathfrak{U}} K(\mathfrak{U})$, where $\mathfrak{U}$ runs through all the relatively compact open subsets of $\mathfrak{X}$. Furthermore, if $\mathfrak{Z}$ is a closed subset of $\mathfrak{X}$ and $\mathcal{F}$ is supported in $\mathfrak{Z}$, then the topological class of $\mathcal{F}$ lies naturally in $\lim _{\mathfrak{U}} K_{\mathfrak{U} \cap \mathfrak{Z}}(\mathfrak{U})$. 
We are going to prove two fundamental properties of the topological class, namely the functoriality by pullback and the homotopy invariance. We start with the first one.

Proposition 3.20 Let $f$ be a weak morphism between two relative analytic spaces $\mathfrak{X}$ and $\mathfrak{X}^{\prime}, \mathfrak{Z}$ be a relative analytic subspace of $\mathfrak{X}^{\prime}$ and $\mathcal{F}$ be a relatively coherent sheaf on $\mathfrak{X}^{\prime}$ supported in $\mathfrak{Z}$. Then the pullback morphism

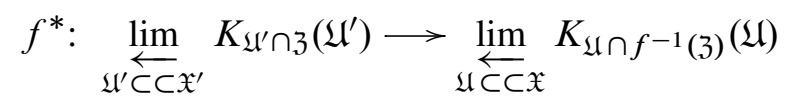

$\operatorname{maps}\left[\mathcal{F}^{\infty}\right]$ to $\left[\left(f^{!} \mathcal{F}\right)^{\infty}\right]$.

Proof We can assume that $\mathcal{F}^{\infty}$ admits a global locally free resolution on $\mathfrak{X}^{\prime}$. Let $\left(E_{i}\right)_{0 \leq i \leq N}$ be such a resolution. Then for every nonnegative integer $k$, the cohomology sheaf of $f^{*} E_{\bullet}$ in degree $k$ is

which is isomorphic to

$$
\operatorname{Tor}_{f}^{k}{ }^{-1} \mathcal{C}_{\mathfrak{X}^{\prime}}^{\infty}\left(f^{-1} \mathcal{F}^{\infty}, \mathcal{C}_{\mathfrak{X}}^{\infty}\right)
$$

$$
\mathcal{T o r}_{f^{-1}}^{k} \mathcal{O}_{\mathfrak{X}^{\prime}}^{\text {rel }}\left(f^{-1} \mathcal{F}, \mathcal{O}_{\mathfrak{X}}^{\text {rel }}\right)^{\infty}
$$

by Lemma 3.5 and Lemma 3.18. We define the sheaves $\mathcal{N}_{k}$ and $\mathcal{I}_{k}$ by the expressions $\mathcal{N}_{k}=\operatorname{ker}\left(f^{*} E_{k} \rightarrow f^{*} E_{k-1}\right)$ and $\mathcal{I}_{k}=\operatorname{Im}\left(f^{*} E_{k+1} \rightarrow f^{*} E_{k}\right)$. Then we have exact sequences

$$
\begin{aligned}
& 0 \longrightarrow \mathcal{N}_{k} \longrightarrow f^{*} E_{k} \longrightarrow \mathcal{I}_{k-1} \longrightarrow 0 \\
& 0 \longrightarrow \mathcal{I}_{k} \longrightarrow \mathcal{N}_{k} \longrightarrow \mathcal{T} r_{f^{-1}}^{k} \mathcal{C}_{\mathfrak{X}^{\prime}}^{\infty}\left(f^{-1} \mathcal{F}^{\infty}, \mathcal{C}_{\mathfrak{X}}^{\infty}\right) \longrightarrow 0 .
\end{aligned}
$$

If $\mathfrak{U}$ is relatively compact in $\mathfrak{X}$, then the sheaves

$$
\mathcal{T o r}_{f}^{i}{ }^{-1} \mathcal{C}_{\mathfrak{X}^{\prime}}^{\infty}\left(f^{-1} \mathcal{F}^{\infty}, \mathcal{C}_{\mathfrak{X}}^{\infty}\right)
$$

admit, by Proposition 3.19, a global locally free resolution on $\mathfrak{U}$. Since $\mathcal{N}_{0}=E_{0}$, the repeated use of Proposition 3.17 (iv) with the exact sequences (3-3) and (3-4) shows that the sheaves $\mathcal{N}_{i}$ and $\mathcal{I}_{i}$ admit global locally free resolutions on $\mathfrak{U}$, and that the following identities hold in $K_{\mathfrak{U} \cap f^{-1}(\mathfrak{Z})}(\mathfrak{U})$ :

$$
\left[f^{*} E_{k}\right]=\left[\mathcal{N}_{k}\right]+\left[\mathcal{I}_{k-1}\right] \quad \text { and } \quad\left[\mathcal{N}_{k}\right]=\left[\mathcal{I}_{k}\right]+\left[\mathcal{T}_{\text {or }}^{k}{ }^{-1} \mathcal{C}_{\mathfrak{X}^{\prime}}^{\infty}\left(f^{-1} \mathcal{F}^{\infty}, \mathcal{C}_{\mathfrak{X}}^{\infty}\right)\right] \text {. }
$$

As a consequence, $\sum_{i=0}^{N}(-1)^{i}\left[f^{*} E_{i}\right]=\sum_{i=0}^{N}(-1)^{i}\left[\mathcal{T o r}_{f^{-1}}^{i} \mathcal{C}_{\mathfrak{X}^{\prime}}^{\infty}\left(f^{-1} \mathcal{F}^{\infty}, \mathcal{C}_{\mathfrak{X}}^{\infty}\right)\right]$. This yields the result.

We can now come to the homotopy invariance of the topological class: 
Proposition 3.21 Let $\mathfrak{Y}$ be a relative analytic space over $B \times[0,1]$, $\mathfrak{R}$ be a relative analytic subspace of $\mathfrak{Y}$ and $\mathcal{F}$ be a relatively coherent sheaf on $\mathfrak{Y}$ supported in $\mathfrak{R}$. Assume that the pair $(\mathfrak{R}, \mathfrak{Y})$ is topologically trivial over $[0,1]$ (ie for any $t_{0}$ in $[0,1]$, if $\mathfrak{X}=\mathfrak{Y}_{t_{0}}$ and $\mathfrak{Z}=\mathfrak{R}_{t_{0}}$, then there exists a homeomorphism between $\mathfrak{Y}$ and $\mathfrak{X} \times[0,1]$ over $B \times[0,1]$ mapping $\mathfrak{R}$ to $\mathfrak{Z} \times[0,1])$. For every $t$ in $[0,1]$, let $i_{t}: \mathfrak{Y}_{t} \longrightarrow \mathfrak{Y}$ be the natural inclusion. Then, via the homeomorphism between $\mathfrak{Y}_{t}$ and $\mathfrak{X}$, the topological class $\left[\left(i_{t}^{*} \mathcal{F}\right)^{\infty}\right]$ in $\lim _{\mathfrak{U} \subset \subset \mathfrak{X}} K_{\mathfrak{U} \cap \mathfrak{Z}}(\mathfrak{U})$ is independent of $t$.

Proof Let $\mathfrak{U}$ be an open relatively compact subset of $\mathfrak{X}, \varphi: \mathfrak{X} \times[0,1] \longrightarrow \mathfrak{Y}$ be a homeomorphism trivializing the pair $(\mathfrak{R}, \mathfrak{Y})$ and let $\mathfrak{U}^{\prime}=\varphi(\mathfrak{U} \times[0,1])$. Then $\mathfrak{U}^{\prime}$ is open and relatively compact in $\mathfrak{Y}$. We take a locally free resolution $\left(E_{i}\right)_{0 \leq i \leq N}$ of $\mathcal{F}^{\infty}$ on $\mathfrak{U}^{\prime}$. By the homotopy invariance property for topological $K$-theory, the class $\sum_{i=0}^{N}(-1)^{i}\left[i_{t}^{*} \mathcal{F}^{\infty}\right]$ in $K_{\mathfrak{U} \cap \mathfrak{Z}}(\mathfrak{U})$ is independent of $t$. By Proposition 3.16 (vi) and Proposition $3.20, i_{t}^{*} E_{\bullet}$ is a locally free resolution of $i_{t}^{*} \mathcal{F}^{\infty}$ in $\mathfrak{U}$. This yields the desired result.

\subsection{Relative incidence sheaves}

Let $(X, J)$ be an almost-complex compact four-manifold and $n$ be a fixed positive integer. If $W, W^{\prime}$ and $W^{\prime \prime}$ are small neighbourhoods of the incidence loci $Z_{n \times 1}, Z_{n}$ and $Z_{n+1}$ introduced in (2-5) and (2-6), let $J_{n \times 1}^{\text {rel }}, J_{n}^{\text {rel }}$ and $J_{n+1}^{\text {rel }}$ be relative integrable complex structures on $W, W^{\prime}$ and $W^{\prime \prime}$ respectively. To simplify the notation, we put

$$
\begin{aligned}
X^{[n]} & =X_{J_{n}^{\text {rel }}}^{[n]}, & X^{[n+1]} & =X_{J_{n+1}^{\text {rel }}}^{[n+1]}, \\
X^{[n+1, n]} & =X_{J_{n \times 1}^{[n+1, n]}}^{[n+1,1}, & X^{[n] \times[1]} & =\left(X^{[n] \times[1]}, J_{n \times 1}^{\text {rel }}, J_{n \times 1}^{\text {rel }}\right) .
\end{aligned}
$$

Definition 3.22 The four relative incidence sets $\mathfrak{Y}_{n}, \mathfrak{Y}_{n+1}, \widetilde{\mathfrak{Y}}_{n}$ and $\widetilde{\mathfrak{Y}}_{n+1}$ are defined by:

$$
\begin{aligned}
\mathfrak{Y}_{n} & =\left\{(\xi, w ; \underline{x}) \text { in } W_{\text {rel }}^{\prime[n]} \times_{X^{(n)}} W^{\prime} \text { such that } w \in \operatorname{supp}(\xi)\right\}, \\
\mathfrak{Y}_{n+1} & =\left\{(\xi, w ; \underline{y}) \text { in } W_{\text {rel }}^{\prime \prime[1]} \times_{X^{(n+1)}} W^{\prime \prime} \text { such that } w \in \operatorname{supp}(\xi)\right\}, \\
\tilde{\mathfrak{Y}}_{n} & =\left\{(\xi, w ; \underline{x}, p) \text { in } W_{\text {rel }}^{[n]} \times_{X^{(n)} \times X} W \text { such that } w \in \operatorname{supp}(\xi)\right\}, \\
\tilde{\mathfrak{Y}}_{n+1} & =\left\{(\xi, w ; \underline{x}, p) \text { in } W_{\text {rel }}^{[n+1]} \times_{X^{(n)} \times X} W \text { such that } w \in \operatorname{supp}(\xi)\right\} .
\end{aligned}
$$

The relative incidence sets $\mathfrak{Y}_{n}, \mathfrak{Y}_{n+1}, \widetilde{\mathfrak{Y}}_{n}$ and $\tilde{\mathfrak{Y}}_{n+1}$ are relative analytic subspaces of $W_{\text {rel }}^{\prime[n]} \times_{X^{(n)}} W^{\prime}, W_{\text {rel }}^{\prime \prime[1]}{ }^{[n+1]} \times_{X^{(n+1)}} W^{\prime \prime}, W_{\text {rel }}^{[n]} \times_{X^{(n)} \times X} W$ and $W_{\text {rel }}^{[n+1]} \times_{X^{(n)} \times X} W$ respectively. For instance, if $\left\{\phi_{i}: U_{i} \stackrel{\sim}{\longrightarrow} \Omega_{i} \times V_{i}\right\}_{i \in I}$, where $V_{i}$ and $\Omega_{i}$ are open 
subsets of $X^{(n)}$ and $\mathbb{C}^{2}$ respectively, is a maximal relative atlas on $W^{\prime}$, the associated atlas on $W_{\text {rel }}^{\prime[n]} \times_{X^{(n)}} W^{\prime}$ is the completed atlas of

$$
\left\{\phi_{i}^{[n]} \times_{V_{i}} \phi_{i}: U_{i}^{[n]} \times_{V_{i}} U_{i} \stackrel{\sim}{\longrightarrow} \Omega_{i}^{[n]} \times \Omega_{i} \times V_{i}\right\}_{i \in I} .
$$

For any $i$ in $I$, let $Y_{n, i}$ be the incidence locus in $\Omega_{i}^{[n]} \times \Omega_{i}$ defined by (2-1). Then we have $\left(\phi_{i}^{[n]} \times_{V_{i}} \phi_{i}\right)\left[\left(U_{i}^{[n]} \times_{V_{i}} U_{i}\right) \cap \mathfrak{Y}_{n}\right]=Y_{n, i} \times V_{i}$.

Definition 3.23 (i) The relative exceptional divisor $D_{\text {rel }}$ is the subset of $W_{\text {rel }}^{[n+1, n]}$ defined by

$$
D_{\text {rel }}=\left\{\left(\xi, \xi^{\prime} ; \underline{x}, p\right) \text { in } W_{\text {rel }}^{[n+1, n]} \text { such that } \operatorname{supp}(\xi)=\operatorname{supp}\left(\xi^{\prime}\right)\right\} .
$$

(ii) The relative residual morphism $\bar{\rho}: W_{\mathrm{rel}}^{[n+1, n]} \longrightarrow W$ is defined by

$$
\bar{\rho}\left(\xi, \xi^{\prime} ; \underline{x}, p\right)=\left(\operatorname{supp}\left(\xi / \xi^{\prime}\right) ; \underline{x}, p .\right)
$$

(iii) The relative diagonal $\Delta_{\text {rel }}$ is the subset of $W \times_{X^{(n)} \times X} W$ defined by

$$
\Delta_{\text {rel }}=\left\{\left(w_{1}, w_{2} ; \underline{x}, p\right) \text { in } W \times_{X^{(n)} \times X} W \text { such that } w_{1}=w_{2}\right\} .
$$

The set $D_{\text {rel }}$ is a relative analytic subspace of $W_{\text {rel }}^{[n+1, n]}$ of relative codimension one, and the fibers $D_{\text {rel, }, x}, p$ of $D_{\text {rel }}$ over $X^{(n)} \times X$ are the usual exceptional divisors in $\left.W_{\underline{x}}^{[n+1, p}, n\right]$ defined by (2-2). The ideal sheaf $\mathcal{J}_{D_{\text {rel }}}^{\text {rel }}$ is locally free of rank one on $W_{\text {rel }}^{[n+1, n]}$, so that $\mathcal{J}_{D_{\text {rel }}}^{\infty}$ is a complex line bundle on $W_{\text {rel }}^{[n+1, n]}$.

Let us give a list of notation which are extensively used in the sequel of this section as well as in Section 5.2 and Section 6.

Notation 3.24 Notation related to sheaves is as follows:

- The relatively coherent sheaves

$$
\begin{gathered}
\mathcal{O}_{\mathfrak{Y} n}^{\text {rel }}, \mathcal{O}_{\mathfrak{Y}_{n+1}}^{\text {rel }}, \mathcal{O}_{\widetilde{\mathfrak{Y}}_{n}}^{\text {rel }}, \mathcal{O}_{\mathfrak{Y}_{n+1}}^{\text {rel }} \quad\left(\text { resp. } \mathcal{J}_{\mathfrak{Y}_{n}}^{\text {rel }}, \mathcal{J}_{\mathfrak{Y}_{n+1}}^{\text {rel }}, \mathcal{J}_{\widetilde{\mathfrak{Y}}_{n}}^{\text {rel }}, \mathcal{J}_{\widetilde{\mathfrak{Y}}_{n+1}}^{\text {rel }}\right) \\
\text { on } W_{\text {rel }}^{\prime[n]} \times_{X^{(n)}} W^{\prime}, W_{\text {rel }}^{\prime \prime[1]} \times_{X^{(n+1)}} W^{\prime \prime}, W_{\text {rel }}^{[n+1} \times_{X^{(n)} \times X} W, W_{\text {rel }}^{[n+1]} \times_{X^{(n)} \times X} W
\end{gathered}
$$

defined by Proposition 3.11 are denoted by $\mathcal{O}_{n}, \mathcal{O}_{n+1}, \widetilde{\mathcal{O}}_{n}$ and $\widetilde{\mathcal{O}}_{n+1}$ (resp. $\mathcal{J}_{n}, \mathcal{J}_{n+1}, \widetilde{\mathcal{J}}_{n}$ and $\left.\widetilde{\mathcal{J}}_{n+1}\right)$.

- The ideal sheaf $\mathcal{J}_{D_{\text {rel }}}^{\text {rel }}$ is denoted by $\mathcal{L}$.

- The relatively coherent sheaf $\mathcal{O}_{\Delta_{\text {rel }}}^{\text {rel }}$ on $W \times_{X^{(n)} \times X} W$ is denoted by $\mathcal{O}_{\Delta}$. 
Notation for cohomology classes is given as follows:

- The restriction to $X^{[n+1, n]}$ of the first Chern class of $\mathcal{L}^{\infty}$ in $H^{2}\left(X^{[n+1, n]}, \mathbb{Q}\right)$ is denoted by $l$.

- If $0 \leq i \leq 2 n+2$, we define a class $\mu_{i, n}$ in $H^{2 i}\left(X^{[n]} \times X\right.$, $\left.\mathbb{Q}\right)$ by the formula $\mu_{i, n}=\left.c_{i}\left(\left[\tilde{\mathcal{O}}_{n}^{\infty}\right]\right)\right|_{X^{[n] \times[1]}}$.

For morphisms, we use the following notation:

- The natural morphisms from $W_{\text {rel }}^{[n+1, n]}$ to $W_{\text {rel }}^{[n]}$ and $W_{\text {rel }}^{[n+1]}$ are denoted by $\phi$ and $\psi$ respectively.

- The natural projections from

$$
\begin{gathered}
W_{\text {rel }}^{\prime[n]} \times_{X^{(n)}} W^{\prime}, \quad W_{\text {rel }}^{\prime \prime[n+1]} \times_{X^{(n+1)}} W^{\prime \prime}, \quad W_{\text {rel }}^{[n]} \times_{X^{(n)} \times X} W, \\
W_{\text {rel }}^{[n+1]} \times_{X^{(n)} \times X} W, \quad W_{\text {rel }}^{[n+1, n]} \times_{X^{(n)} \times X} W \\
W_{\text {rel }}^{\prime[n]}, W_{\text {rel }}^{\prime \prime[n+1]}, W_{\text {rel }}^{[n]}, W_{\text {rel }}^{[n+1]}, W_{\text {rel }}^{[n+1, n]}, \text { resp. }
\end{gathered}
$$

to

are denoted by $\bar{p}, \bar{q}, p, q, \tilde{p}$, resp.

- The morphism $\bar{\rho}: W_{\text {rel }}^{[n+1, n]} \longrightarrow W$ has already been defined in Definition 3.23 (ii).

- The morphism $\bar{\sigma}: W_{\text {rel }}^{[n+1, n]} \longrightarrow W_{\text {rel }}^{[n]} \times_{X^{(n)} \times X} W$ is defined by $\bar{\sigma}=(\phi, \bar{\rho})$.

- The morphism $j: W_{\text {rel }}^{[n+1, n]} \longrightarrow W_{\text {rel }}^{[n+1, n]}{ }_{X^{(n)} \times X} W$ is defined by $j=(\mathrm{id}, \bar{\rho})$.

- If $f: \mathfrak{X} \longrightarrow \mathfrak{X}^{\prime}$ is a morphism of relative analytic spaces over $X^{(n)} \times X$, we define $f_{W}$ by

$$
f_{W}=f \times_{X^{(n)} \times X} \operatorname{id}_{W}: \mathfrak{X} \times_{X^{(n)} \times X} W \longrightarrow \mathfrak{X}^{\prime} \times_{X^{(n)} \times X} W .
$$

The relative incidence sheaves $\mathcal{O}_{n}, \mathcal{O}_{n+1}, \widetilde{\mathcal{O}}_{n}$ and $\widetilde{\mathcal{O}}_{n+1}$ are related if the relative integrable complex structures $J_{n}^{\text {rel }}, J_{n+1}^{\text {rel }}$ and $J_{n \times 1}^{\text {rel }}$ satisfy some compatibility conditions.

- If the compatibility condition (A) of Section 2.4 is satisfied in the case $m=n+1$, let $k: W_{\text {rel }}^{\prime n]} \times_{X^{(n)}}\left(X^{(n)} \times X\right) \hookrightarrow W_{\text {rel }}^{[n]}$ be the associated injection. Letting $r$ denote the base change morphism from $W_{\mathrm{rel}}^{\prime[n]} \times_{X^{(n)}}\left(X^{[n]} \times X\right)$ to $W_{\mathrm{rel}}^{\prime[n]}$, then $r^{*} \mathcal{O}_{n}=k^{*} \widetilde{\mathcal{O}}_{n}$ and $r^{*} \mathcal{J}_{n}=k^{*} \tilde{\mathcal{J}}_{n}$.

- If the condition (B) of Section 2.4 is satisfied, a weak morphism $s$ from $W_{\text {rel }}^{[n+1]}$ to $W_{\text {rel }}^{\prime \prime[n+1]}$ can be obtained by composing the isomorphism

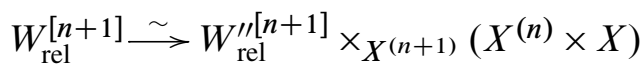

with the base change morphism from $W_{\text {rel }}^{\prime \prime[n+1]} \times_{X^{(n+1)}}\left(X^{(n)} \times X\right)$ to $W_{\text {rel }}^{\prime \prime[n+1]}$. Then $s^{*} \mathcal{O}_{n+1}=\widetilde{\mathcal{O}}_{n+1}$ and $s^{*} \mathcal{J}_{n+1}=\widetilde{\mathcal{J}}_{n+1}$. 
The sheaves $\widetilde{\mathcal{O}}_{n}$ and $\widetilde{\mathcal{O}}_{n+1}$ fit into an important exact sequence:

Proposition 3.25 Let $\mathfrak{X}$ be the relative analytic space $W_{\text {rel }}^{[n+1, n]} \times X_{X^{(n)} \times X} W$.

(i) There is a natural exact sequence on $\mathfrak{X}$ :

$$
0 \longrightarrow j_{*} \mathcal{L} \longrightarrow \psi_{W}^{*} \tilde{\mathcal{O}}_{n+1} \longrightarrow \phi_{W}^{*} \tilde{\mathcal{O}}_{n} \longrightarrow 0 .
$$

(ii) The relatively coherent sheaves $j_{*} \mathcal{L}$ and $\tilde{p}^{*} \mathcal{L} \otimes \bar{\rho}_{W}^{*} \mathcal{O}_{\Delta}$ are isomorphic on $\mathfrak{X}$.

(iii) The three sheaves

$$
\mathcal{T o r}_{\bar{\rho}_{W}^{-1}}^{i} \mathcal{O}_{W}^{\text {rel }}\left(\bar{\rho}_{W}^{1} \mathcal{O}_{\Delta}, \mathcal{O}_{\mathfrak{X}}^{\text {rel }}\right), \quad \mathcal{T}_{o r}^{i} \psi_{W}^{-1} \mathcal{O}^{\text {rel }}\left(\psi_{W}^{-1} \widetilde{\mathcal{O}}_{n+1}, \mathcal{O}_{\mathfrak{X}}^{\text {rel }}\right)
$$

and

$$
\mathcal{T}_{o r}^{i} \phi_{W}^{-1} \mathcal{O}^{\text {rel }}\left(\phi_{W}^{-1} \widetilde{\mathcal{O}}_{n}, \mathcal{O}_{\mathfrak{X}}^{\text {rel }}\right)
$$

vanish for any positive integer $i$.

Proof Using Proposition 3.6, the proposition is an immediate consequence of the analogous results in the integrable case. For instance, the exact sequence of (i) is obtained via relative holomorphic charts on $W$ using the exact sequence (2-3).

We now turn to the computation of the classes $\mu_{i, n}$.

Lemma 3.26 For every positive integer $i$, the classes $\mu_{i, n}$ are independent of the relative integrable complex structure $J_{n \times 1}^{\mathrm{rel}}$.

Proof Let $J_{0, n \times 1}^{\text {rel }}$ and $J_{1, n \times 1}^{\text {rel }}$ be two relative integrable complex structures on a neighbourhood $W$ of $Z_{n \times 1}$ such that $\left\|J_{0, n \times 1}^{\mathrm{rel}}-J\right\|_{C^{0}, g, W}$ and $\left\|J_{1, n \times 1}^{\mathrm{rel}}-J\right\|_{C^{0}, g, W}$ are strictly smaller than the bound $\varepsilon_{0}$ given in Proposition 2.13. Then there exist a neighbourhood $U$ of $Z_{n \times 1}$ included in $W$ as well as a relative integrable complex structure $\widetilde{J}_{n \times 1}^{\text {rel }}$ on $U \times[0,1]$ (considered as a relative differentiable space over the base $\left.X^{(n)} \times X \times[0,1]\right)$ such that $\left.\widetilde{J}_{n \times 1}^{\text {rel }}\right|_{U \times\{0\}}=\left.J_{0, n \times 1}^{\text {rel }}\right|_{U}$ and $\left.\widetilde{J}_{n \times 1}^{\text {rel }}\right|_{U \times\{1\}}=\left.J_{1, n \times 1}^{\text {rel }}\right|_{U}$. If $\mathfrak{X}=\left(U \times[0,1], \widetilde{J}_{n \times 1}^{\text {rel }}\right)$, let us introduce the incidence set

$$
\hat{\mathfrak{Y}}_{n}=\left\{(\xi, w ; \underline{x}, p, t) \text { in } \mathfrak{X}^{[n]} \times_{X^{(n)} \times X \times[0,1]} \mathfrak{X} \text { such that } w \in \operatorname{supp} \xi\right\} .
$$

Then $\hat{\mathfrak{Y}}_{n}$ is a relative analytic subset of $\mathfrak{X}^{[n]} \times_{X^{(n)} \times X \times[0,1]} \mathfrak{X}$. Furthermore, for any $t$ in $[0,1]$, if $i_{t}: \mathfrak{X}_{t}^{[n]} \times_{X^{(n)} \times X \times\{t\}} \mathfrak{X}_{t} \longrightarrow \mathfrak{X}^{[n]} \times_{X^{(n)} \times X \times[0,1]} \mathfrak{X}$ is the natural injection, then $i_{t}^{*} \mathcal{O}_{\widehat{\mathfrak{Y}}_{n}}^{\text {rel }}$ is the incidence sheaf $\widetilde{\mathcal{O}}_{n}$ on $U^{[n]} \times_{X^{(n)} \times X} U$, where $U$ is endowed with the relative integrable complex structure $\left.\widetilde{J}_{n \times 1}^{\text {rel }}\right|_{U \times\{t\}}$. Since the relative product Hilbert scheme $\left(X^{[n] \times[1]}, \tilde{J}_{n \times 1}^{\text {rel }}\right)$ is a topological fibration over $[0,1]$, the required result is a direct consequence of Proposition 3.21. 
We compute now the cohomology classes $\mu_{i, n}$. Let $\rho: X^{[n+1, n]} \longrightarrow X$ be the restriction of $\operatorname{pr}_{1} \circ \bar{\rho}$ to $X^{[n+1, n]}$. If $\lambda$ is the canonical homotopy class in $\left[X^{[n+1, n]}: X^{[n]}\right]$ introduced in Section 2.4, we define $\sigma$ in $\left[X^{[n+1, n]}: X^{[n]} \times X\right]$ by $\sigma=(\lambda, \rho)$.

Proposition 3.27 If $1 \leq i \leq 2 n+2, \mu_{i, n}=(-1)^{i} \sigma_{*}\left(l^{i}\right)$.

Proof Let us assume that $\left(W, J_{n \times 1}^{\text {rel }}\right)$ and $\left(W^{\prime}, J_{n}^{\text {rel }}\right)$ satisfy the compatibility condition (A) of Section 2.4 for $m=n+1$. Then $X^{[n] \times[1]}=X^{[n]} \times X$. In this proof, we denote the two relative analytic spaces $W_{\text {rel }}^{[n+1, n]}$ and $W_{\text {rel }}^{[n]} \times_{X^{(n)} \times X} W$ by $\mathfrak{X}$ and $\mathfrak{X}^{\prime}$ respectively. The homotopy class of the restriction of $\bar{\sigma}: \mathfrak{X} \longrightarrow \mathfrak{X}^{\prime}$ to $X^{[n+1, n]}$ is $\sigma$.

Let $\left\{\phi_{i}: U_{i} \stackrel{\sim}{\longrightarrow} \Omega_{i} \times V_{i}\right\}_{i \in I}$, where $\Omega_{i}$ and $V_{i}$ are open subsets of $\mathbb{C}^{2}$ and $X^{(n)} \times X$ respectively, be a maximal relative holomorphic atlas on $W$. If $\psi_{i}=\phi_{i}^{[n+1, n]}$, if $\gamma_{i}=\phi_{i}^{[n]} \times_{V_{i}} \phi_{i}$ and if $O_{i}=U_{i, \text { rel }}^{[n]} \times_{V_{i}} U_{i}$, then the family

$$
\left\{\psi_{i}: U_{i, \text { rel }}^{[n+1, n]} \stackrel{\sim}{\longrightarrow} \Omega_{i}^{[n+1, n]} \times V_{i}\right\}_{i \in I} \quad\left(\text { resp. }\left\{\gamma_{i}: O_{i} \stackrel{\sim}{\longrightarrow} \Omega_{i}^{[n]} \times \Omega_{i} \times V_{i}\right\}_{i \in I}\right)
$$

is a relative holomorphic atlas on $\mathfrak{X}$ (resp. $\mathfrak{X}^{\prime}$ ). For any $i$ in $I$, let $Y_{n, i}$ be the incidence locus in $\Omega_{i}^{[n]} \times \Omega_{i}$ defined by (2-2), and let $0 \longrightarrow \mathcal{A}_{i} \longrightarrow \mathcal{B}_{i}$ be a locally free resolution of length two of the ideal sheaf $\mathcal{J}_{Y_{n, i}}$ (see Section 2.1). By the very construction of global smooth resolutions for relatively coherent sheaves (Proposition 3.19), we can assume, after shrinking $W$ if necessary, that there exists a locally free resolution $0 \longrightarrow \mathcal{A} \longrightarrow \mathcal{B}$ of length 2 of $\tilde{\mathcal{J}}_{n}^{\infty}$ on $\mathfrak{X}^{\prime}$ such that for any $i$ in $I$, $0 \longrightarrow \gamma_{i}^{-1} \mathcal{A}_{i}^{\infty} \longrightarrow \gamma_{i}^{-1} \mathcal{B}_{i}^{\infty}$ is a subresolution of $\left.\left.0 \longrightarrow \mathcal{A}\right|_{O_{i}} \longrightarrow \mathcal{B}\right|_{O_{i}}$, where by a slight abuse of notation, we write $\mathcal{A}_{i}^{\infty}$ instead of

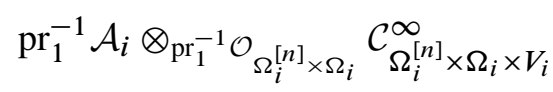

and $\mathcal{B}_{i}^{\infty}$ instead of

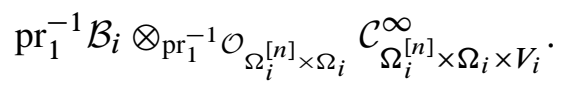

Let $\mathbb{P}(\mathcal{B})$ be the projective bundle of $\mathcal{B}$ (using Grothendieck's convention), $\mathcal{K}$ be the universal quotient line bundle on $\mathbb{P}(\mathcal{B}), \pi: \mathbb{P}(\mathcal{B}) \longrightarrow W_{\text {rel }}^{[n]} \times_{X^{(n)} \times X} W$ be the projection and $s$ be the section of $\pi^{*} \mathcal{A}^{*} \otimes \mathcal{K}$ obtained via the morphism $\pi^{*} \mathcal{A} \longrightarrow \pi^{*} \mathcal{B} \longrightarrow \mathcal{K}$.

Lemma 3.28 (i) The vanishing locus $Z(s)$ of $s$ is canonically isomorphic to $\mathfrak{X}$.

(ii) After changing base from $X^{(n)} \times X$ to $X^{n} \times X$, the section $s$ is transverse to the zero section.

(iii) If $j$ is the embedding of $\mathfrak{X}$ into $\mathbb{P}(\mathcal{B})$, then $j^{*} \mathcal{K} \simeq \mathcal{L}^{\infty}$. 
Proof (i) By Proposition 3.17 (i), we can split the injection of $\gamma_{i}^{-1} \mathcal{A}_{i}^{\infty}$ in $\left.\mathcal{A}\right|_{O_{i}}$. If $\left.\mathcal{A}\right|_{O_{i}} \simeq \gamma_{i}^{-1}\left(\mathcal{A}_{i}^{\infty} \oplus \mathcal{R}_{i}\right)$ is such a splitting, it induces another splitting $\left.\mathcal{B}\right|_{o_{i}} \simeq$ $\gamma_{i}^{-1}\left(\mathcal{B}_{i}^{\infty} \oplus \mathcal{R}_{i}\right)$. The resolution $\left.\left.0 \longrightarrow \mathcal{A}\right|_{O_{i}} \longrightarrow \mathcal{B}\right|_{O_{i}}$ is therefore isomorphic to

where

$$
\begin{gathered}
0 \longrightarrow \gamma_{i}^{-1}\left(\mathcal{A}_{i}^{\infty} \oplus \mathcal{R}_{i}\right) \stackrel{N_{i}}{\longrightarrow} \gamma_{i}^{-1}\left(\mathcal{B}_{i}^{\infty} \oplus \mathcal{R}_{i}\right), \\
N_{i}=\left(\begin{array}{cc}
\gamma_{i}^{-1} M_{i} & 0 \\
0 & \mathrm{id}
\end{array}\right) .
\end{gathered}
$$

For any point $p$ in $\Omega_{i}^{[n+1, n]} \times V_{i}$, we have an obvious bijection between the two sets

$$
\left\{u \text { in }\left(\mathcal{B}_{i \mid p}^{\infty} \oplus \mathcal{R}_{i \mid p}\right)^{*} \text { such that } u \text { vanishes on }\left(\mathcal{A}_{i \mid p}^{\infty} \oplus \mathcal{R}_{i \mid p}\right)\right\}
$$

and

$$
\left\{u \text { in }\left(\mathcal{B}_{i \mid p}^{\infty}\right)^{*} \text { such that } u \text { vanishes on } \mathcal{A}_{i \mid p}^{\infty}\right\} \text {. }
$$

If we consider the embedding of $\mathbb{P}\left(\gamma_{i}^{-1} \mathcal{B}_{i}^{\infty}\right)$ in $\mathbb{P}\left(\left.\mathcal{B}\right|_{O_{i}}\right)$ given by the splitting of $\left.\mathcal{B}\right|_{O_{i}}$, this means that $Z(s) \cap \pi^{-1}\left(O_{i}\right)$ lies in $\mathbb{P}\left(\gamma_{i}^{-1} \mathcal{B}_{i}^{\infty}\right)$. Furthermore, it is easy to see that the embedding of $Z(s) \cap \pi^{-1}\left(O_{i}\right)$ in $\mathbb{P}\left(\gamma_{i}^{-1} \mathcal{B}_{i}^{\infty}\right)$ is independent of the splitting. If $\tilde{\pi}: \mathbb{P}\left(\mathcal{B}_{i}\right) \longrightarrow \Omega_{i}^{[n]} \times \Omega_{i}$ is the projection of the projective bundle of $\mathcal{B}_{i}$, if $\tilde{s}$ is the section of $\tilde{\pi}^{*} \mathcal{A}_{i}^{*}(1)$ given by the morphism $\tilde{\pi}^{*} \mathcal{A}_{i} \longrightarrow \tilde{\pi}^{*} \mathcal{B}_{i} \longrightarrow \mathcal{O}_{\mathbb{P}\left(\mathcal{B}_{i}\right)}(1)$ and if $\delta_{i}: \mathbb{P}\left(\gamma_{i}^{-1} \mathcal{B}_{i}^{\infty}\right) \stackrel{\sim}{\longrightarrow} \mathbb{P}\left(\mathcal{B}_{i}^{\infty}\right)$ is the natural isomorphism induced by $\gamma_{i}$, then $Z(s) \cap \pi^{-1}\left(O_{i}\right)=\delta_{i}^{-1}\left(Z(\widetilde{s}) \times V_{i}\right)$. The zero locus $Z(\widetilde{s})$ being canonically isomorphic to $\Omega_{i}^{[n+1, n]}$, we get a commutative diagram

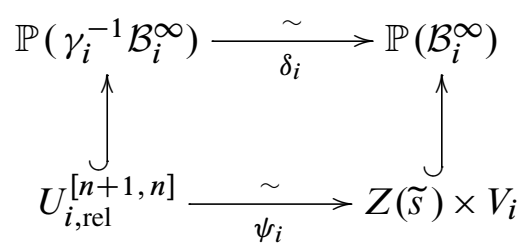

Thus $Z(s) \cap \pi^{-1}\left(O_{i}\right) \simeq U_{i, \text { rel }}^{[n+1, n]}$.

(ii) Let $p$ be in $\left(\Omega_{i}^{[n]} \times \Omega_{i}\right) \times V_{i}$. We choose a neighbourhood $U_{p} \times V_{p}$ of $p$ such that $\mathcal{A}_{i}, \mathcal{B}_{i}$ and $\mathcal{R}_{i}$ are trivial on $U_{p}, U_{p}$ and $U_{p} \times V_{p}$, of respective ranks $r, r+1$ and $m$. Let $q$ be a point in $\tilde{\pi}^{-1}(p) \cap\left(Z(\widetilde{s}) \times V_{i}\right)$. We can find an affine hyperplane $H_{1}$ in $\mathbb{C}^{r+1}$ which does not contain zero and an hyperplane $H_{2}$ in $\mathbb{C}^{m}$ such that the open subset $\left(H_{1} \times H_{2} \times U_{p}\right) \times V_{p}$ of $\mathbb{P}\left(\mathcal{B}_{i}^{\infty} \oplus \mathcal{R}_{i}\right)$ contains $q$. The restriction of $s$ to $H_{1} \times\{0\} \times U_{p}$ is a holomorphic map from $H_{1} \times U_{p}$ to $\left(\mathbb{C}^{r}\right)^{*}$ corresponding to the section $\tilde{s}$, and $s:\left(H_{1} \times H_{2} \times U_{p}\right) \times V_{p} \longrightarrow\left(\mathbb{C}^{r} \oplus \mathbb{C}^{m}\right)^{*}$ can be expressed as $s\left(u_{1}, u_{2}, z ; v\right)(\alpha, \beta)=\tilde{s}\left(u_{1}, z\right)(\alpha)+u_{2}(\beta)$. After changing base from $X^{(n)} \times X$ to $X^{n} \times X$, the variable $v$ lies in the preimage of $V_{i}$ in $X^{n} \times X$, which is smooth. Since $\widetilde{s}$ is transverse to the zero section, the result ensues. 
(iii) We write $j$ on $W_{i, \text { rel }}^{[n+1, n]}$ as the composed of the following morphisms:

$$
W_{i, \text { rel }}^{[n+1, n]} \underset{\psi_{i}}{\sim}\left(\Omega_{i}^{[n]} \times \Omega_{i}\right) \times V_{i} \longleftrightarrow \mathbb{P}\left(\mathcal{B}_{i}\right) \times V_{i} \simeq \mathbb{P}\left(\mathcal{B}_{i}^{\infty}\right) \longleftrightarrow \mathbb{P}\left(\left.\mathcal{B}\right|_{O_{i}}\right)
$$

Since the restriction of $\mathcal{O}_{\mathbb{P}\left(\mathcal{B}_{i}\right)}(1)$ to $\Omega_{i}^{[n]} \times \Omega_{i}$ is the ideal sheaf of the exceptional divisor in $\Omega_{i}^{[n]} \times \Omega_{i}$, the sheaves $j^{*} \mathcal{K}$ and $\mathcal{L}^{\infty}$ are isomorphic on $W_{i, \text { rel }}^{[n+1, n]}$. It is then a routine verification to check that these isomorphisms patch together into a global isomorphism between $j^{*} \mathcal{K}$ and $\mathcal{L}^{\infty}$.

We now finish the proof of Proposition 3.27. Let $r: \mathbb{P}(\mathcal{B}) \times_{X^{(n)} \times X}\left(X^{n} \times X\right) \rightarrow \mathbb{P}(\mathcal{B})$ be the natural base change map. Point (ii) of Lemma 3.28 imply that $Z\left(r^{*} s\right)$ is Poincaré dual to the top Chern class of $r^{*}\left(\pi^{*} \mathcal{A}^{*} \otimes \mathcal{K}\right)$. Since the pullback morphism $r^{*}$ is injective in cohomology with rational coefficients, $Z(s)$ is Poincare dual to the top Chern class of $\pi^{*} \mathcal{A}^{*} \otimes \mathcal{K}$ in $H_{8 n+8}(\mathbb{P}(\mathcal{B}), \mathbb{Q})$. Besides, if $\varepsilon$ is the first Chern class of $\mathcal{K}$ in $H^{2}(\mathbb{P}(\mathcal{B}), \mathbb{Q})$, point (iii) of Lemma 3.28 imply that $l=\left.j^{*} \varepsilon\right|_{X^{[n+1, n]}}$. As $\bar{\sigma}$ is proper, we get for $1 \leq i \leq 2 n+2$ :

$$
\begin{aligned}
\bar{\sigma}_{*}\left(j^{*} \varepsilon^{i}\right) & =\pi_{*} j_{*}\left(j^{*} \varepsilon^{i}\right)=\pi_{*}\left([\mathfrak{X}] \cdot \varepsilon^{i}\right) \\
& =\pi_{*}\left(c_{r}\left(\pi^{*} \mathcal{A}^{*} \otimes \mathcal{K}\right) . \varepsilon^{i}\right)=\sum_{k=0}^{r} c_{k}\left(\mathcal{A}^{*}\right) \pi_{*} \varepsilon^{r+i-k} \\
& =\sum_{k=0}^{r} c_{k}\left(\mathcal{A}^{*}\right) s_{i-k}\left(\mathcal{B}^{*}\right)=c_{i}\left(\mathcal{A}^{*}-\mathcal{B}^{*}\right)=(-1)^{i} c_{i}\left(\tilde{\mathcal{J}}_{n}^{\infty}\right)=(-1)^{i} c_{i}\left(\tilde{\mathcal{O}}_{n}^{\infty}\right) .
\end{aligned}
$$

Let $u$ (resp. $v$ ) denote the embedding of $X^{[n+1, n]}\left(\right.$ resp. $\left.X^{[n]} \times X\right)$ in $\mathfrak{X}$ (resp. in $\mathfrak{X}^{\prime}$ ). Since $\bar{\sigma}_{*} \bar{\sigma}^{*}=$ id and $\sigma_{*} \sigma^{*}=$ id, we get

$$
v^{*} \bar{\sigma}_{*}\left(j^{*} \varepsilon^{i}\right)=\sigma_{*} \sigma^{*} v^{*} \bar{\sigma}_{*}\left(j^{*} \varepsilon^{i}\right)=\sigma_{*} u^{*} \bar{\sigma}^{*} \bar{\sigma}_{*}\left(j^{*} \varepsilon^{i}\right)=\sigma_{*} u^{*} j^{*} \varepsilon^{i}=\sigma_{*} l^{i},
$$

so that $\sigma_{*} l^{i}=(-1)^{i} v^{*} c_{i}\left(\widetilde{\mathcal{O}}_{n}^{\infty}\right)=(-1)^{i} \mu_{i, n}$.

\section{The boundary operator}

\subsection{Lehn's formula in the almost-complex case}

Let $(X, J)$ be an almost-complex compact four-manifold, and $W^{\prime}, W$ be respective neighbourhoods of $Z_{n}$ and $Z_{n \times 1}$ endowed with relative integrable complex structures $J_{n}^{\text {rel }}$ and $J_{n \times 1}^{\text {rel }}$. If $\mathcal{L}$ is the invertible sheaf defined in Notation 3.24, let $F$ be the restriction of $\mathcal{L}^{\infty}$ to $X^{[n+1, n]}$. By the homotopy invariance of topological $K$-theory, the class of $F$ in $K\left(X^{[n+1, n]}\right)$ is independent of $J_{n \times 1}^{\mathrm{rel}}$, and its first Chern class is $l$. 
If $\left\{\phi_{i}: U_{i} \stackrel{\sim}{\longrightarrow} Z_{i} \times V_{i}\right\}_{i \in I}$ is a maximal relative holomorphic atlas on $W$, the tautological sheaves

$$
\operatorname{pr}_{1}^{-1} \mathcal{O}_{Z_{i}^{[n]}}^{[n]} \otimes_{\mathrm{pr}_{1}^{-1}} \mathcal{O}_{Z_{i}^{[n]}} \mathcal{O}_{Z_{i}^{\mathrm{rel}} \times V_{i}}^{[n]}
$$

patch together into a locally free sheaf $\mathbb{T}_{\text {rel }}^{[n]}$ on $W_{\text {rel }}^{[n]}$. The restriction $\mathbb{T}^{[n]}$ of $\mathbb{T}_{\text {rel }}^{[n]}$ to $X^{[n]}$ satisfies the properties (see [21, Proposition 5.3, Lemma 5.4, Proposition 5.5]):

(i) The class of $\mathbb{T}^{[n]}$ in $K\left(X^{[n]}\right)$ is independent of $J_{n}^{\text {rel }}$.

(ii) The cohomology class $-2 c_{1}\left(\mathbb{T}^{[n]}\right)$ in $H^{2}\left(X^{[n]}, \mathbb{Q}\right)$ is Poincaré dual to the fundamental homology class of $\partial X^{[n]}$, where

$$
\partial X^{[n]}=\left\{(\xi ; \underline{x}) \text { in } X^{[n]} \text { such that there exists } p \text { in } \underline{x} \text { with length }{ }_{p}(\xi) \geq 2\right\}
$$

is the so-called boundary of $X^{[n]}$.

(iii) If $\lambda$ and $v$ are the homotopy classes introduced in Section 2.4 in the case $m=n+1$, then $v^{*} \mathbb{T}^{[n+1]}-\lambda^{*} \mathbb{T}^{[n]}=F$ in $K\left(X^{[n+1, n]}\right)$.

If $m$ and $n$ are positive integers with $m>n$, we define a class $I_{\mathbb{T}}^{[m, n]}$ in $K\left(X^{[m, n]}\right)$ by the formula $I_{\mathbb{T}}^{[m, n]}=v^{*} \mathbb{T}^{[m]}-\lambda^{*} \mathbb{T}^{[n]}$. Then property (iii) implies that $I_{\mathbb{T}}^{[n+1, n]}$ and $F$ are equal in $K\left(X^{[n+1, n]}\right)$.

Let us recall Lehn's definition of the boundary operator [25, Definition 3.8]:

Definition 4.1 Let $\mathbb{H}=\bigoplus_{n \geq 0} H^{*}\left(X^{[n]}, \mathbb{Q}\right)$.

(i) The boundary operator $\mathfrak{d}: \mathbb{H} \longrightarrow \mathbb{H}$ is defined by

$$
\mathfrak{d}\left[\left(\alpha_{n}\right)_{n \geq 0}\right]=\left(c_{1}\left(\mathbb{T}^{[n]}\right) \cup \alpha_{n}\right)_{n \geq 0} .
$$

(ii) If $A$ is an endomorphism of $\mathbb{H}$, the derivative $A^{\prime}$ of $A$ is defined by the formula

$$
A^{\prime}=[\mathfrak{d}, A]=\mathfrak{d} \circ A-A \circ \mathfrak{d} .
$$

We now state a partial extension of Lehn's main formula [25, Theorem 3.10] for almost-complex four-manifolds.

Theorem 4.2 Let $(X, J)$ be an almost-complex compact four-manifold. Then there exist classes $\left(e_{n}\right)_{n \geq 0}$ in $H^{2}(X, \mathbb{Q})$ such that for all integers $m, n$ and for all rational cohomology classes $\alpha$ and $\beta$ on $X$,

$$
\left[\mathfrak{q}_{n}^{\prime}(\alpha), \mathfrak{q}_{m}(\beta)\right]=-n m \mathfrak{q}_{n+m}(\alpha \beta)+\delta_{n+m, 0}\left(\int_{X} e_{|n|} \alpha \beta\right) \operatorname{id} \mathbb{H}
$$


Proof Exactly as in [25, Lemma 3.9], we start by proving that correspondences actions induced by homology classes on incidence varieties are stable under derivation. We denote by PD the Poincaré duality map between the homology groups and the cohomology groups of a compact topological manifold.

Lemma 4.3 Let $m, n$ be two positive integers with $m>n, u$ be a rational homology class on $X^{[m, n]}$ and $u_{*}: H^{*}\left(X^{[n]}, \mathbb{Q}\right) \longrightarrow H^{*}\left(X^{[m]}, \mathbb{Q}\right)$ be the correspondence map given by the formula $u_{*}(\tau)=\operatorname{PD}^{-1}\left[v_{*}\left(u \cap \lambda^{*} \tau\right)\right]$. Then $\left(u_{*}\right)^{\prime}=\left[u \cap c_{1}\left(I_{\mathbb{T}}^{[m, n]}\right)\right]_{*}$.

Proof For every rational cohomology class $\tau$ on $X^{[n]}$,

$$
\begin{aligned}
\left(u_{*}\right)^{\prime} \tau & =c_{1}\left(\mathbb{T}^{[m]}\right) \cup u_{*} \tau-u_{*}\left(c_{1}\left(\mathbb{T}^{[n]}\right) \cup \tau\right) \\
& =\operatorname{PD}^{-1}\left[\left(v_{*}\left(u \cap \lambda^{*} \tau\right)\right) \cap c_{1}\left(\mathbb{T}^{[m]}\right)-v_{*}\left(u \cap \lambda^{*}\left(c_{1}\left(\mathbb{T}^{[n]}\right) \cup \tau\right)\right)\right] \\
& =\mathrm{PD}^{-1}\left[v_{*}\left(u \cap\left[\left(v^{*} c_{1}\left(\mathbb{T}^{[m]}\right)-\lambda^{*} c_{1}\left(\mathbb{T}^{[n]}\right)\right) \cup \lambda^{*} \tau\right]\right)\right] \\
& =\mathrm{PD}^{-1} v_{*}\left(\left[u \cap c_{1}\left(I_{\mathbb{T}}^{[m, n]}\right)\right] \cap \lambda^{*} \tau\right) \\
& =\left[u \cap c_{1}\left(I_{\mathbb{T}}^{[m, n]}\right)\right]_{*}(\tau) .
\end{aligned}
$$

By this Lemma, the proof of the theorem boils down to computing the commutator of two correspondences. Lehn's proof can be adapted exactly as we did in [21] for the Nakajima relations. This yields (see [22, Section 4.3] for a detailed exposition):

- For all integers $m$ and $n$ such that $m+n$ is nonzero, there exists an excess multiplicity $\mu_{n, m}$ in $\mathbb{Z}$ such that for all rational cohomology classes $\alpha$ and $\beta$ on $X$, the commutation relation $\left[\mathfrak{q}_{n}^{\prime}(\alpha), \mathfrak{q}_{m}(\beta)\right]=\mu_{n, m} \mathfrak{q}_{n+m}(\alpha \beta)$ holds.

- For every nonnegative integer $k$, there exists an excess intersection class $e_{k}$ in $H^{2}(X, \mathbb{Q})$ such that for every integer $n$ and for all rational cohomology classes $\alpha$ and $\beta$ on $X$, the identity $\left[\mathfrak{q}_{n}^{\prime}(\alpha), \mathfrak{q}_{-n}(\beta)\right]=\left(\int_{X} e_{|n|} \alpha \beta\right) \mathrm{id}_{\mathbb{H}}$ holds.

The terms $\mu_{n, m}$ and $e_{k}$ are the excess contributions. The multiplicity $\mu_{n, m}$ can be computed locally on $X$, so that Lehn's proof is valid and gives $\mu_{n, m}=-n m$.

Unlike the multiplicities $\mu_{n, m}$, the excess classes $e_{k}$ involve the global geometry of $X$. We compute these classes in Section 4.3 under the additional assumption that $X$ be symplectic.

Corollary 4.4 When $\alpha$ runs through a basis of $H^{*}(X, \mathbb{Q})$, the operators $\mathfrak{d}$ and $\mathfrak{q}_{1}(\alpha)$ generate $\mathbb{H}$ from the vector 1 .

Proof The corollary is a straightforward consequence of the commutation relations $\left[q_{1}^{\prime}(\alpha), q_{m}(1)\right]=-m q_{m+1}(\alpha), m>0$. 


\subsection{Holomorphic curves in symplectic four-manifolds}

Until now, we have only considered integrable structures in small open sets of $(X, J)$. To compute the excess classes $e_{n}$ appearing in Theorem 4.2, we construct pseudoholomorphic curves in $X$ for perturbed almost-complex structures. To do so we use the following theorem of Donaldson, which is a symplectic analog of Kodaira's embedding theorem:

Theorem 4.5 [11, Theorem 1] Let $(V, \omega)$ be a symplectic manifold of dimension $2 n$ such that $\omega$ is an integral class and let $\widetilde{\omega}$ be a lift of $\omega$ in $H^{2}(V, \mathbb{Z})$. For any sufficiently large positive integer $k$, the Poincaré dual of $k \widetilde{\omega}$ in $H_{2 n-2}(V, \mathbb{Z})$ is the homology class of a closed symplectic submanifold of $V$. More precisely, if $J$ is an almost-complex structure on $V$ compatible with $\omega$, there is a positive constant $C$ such that for any large integer $k$, there exist an almost-complex structure $J_{k}$ on $X$ and a $J_{k}$-holomorphic submanifold $S_{k}$ of codimension two in $V$ such that $k \widetilde{\omega}$ is Poincaré dual to $S_{k}$ and that $\left\|J_{k}-J\right\|_{C^{0}} \leq C / \sqrt{k}$.

We apply this theorem to our situation:

Proposition 4.6 Let $(X, \omega)$ be a symplectic compact four-manifold, $J$ be an adapted almost-complex structure on $X$ and $N$ be the second Betti number of $X$. Then there exist almost-complex structures $\left(J_{i}\right)_{1 \leq i \leq N}$ arbitrary close to $J$ in $C^{0}$-norm and two-dimensional submanifolds $\left(C_{i}\right)_{1 \leq i \leq N}$ such that:

(i) For any $i$ with $1 \leq i \leq N, C_{i}$ is $J_{i}$-holomorphic and $J_{i}$ is integrable in a neighbourhood of $C_{i}$.

(ii) The homology classes of $C_{1}, \ldots, C_{N}$ span $H_{2}(X, \mathbb{Q})$ over $\mathbb{Q}$.

Proof Let $\alpha_{1}, \ldots, \alpha_{N}$ be closed differential two-forms on $X$ such that the $\omega+\alpha_{i}$ 's are rational symplectic forms whose cohomology classes span $H^{2}(X, \mathbb{Q})$. Then there exist almost-complex structures $\left(\widetilde{J}_{i}\right)_{1 \leq i \leq N}$ on $X$ such that for every $i, \widetilde{J}_{i}$ is adapted to $\omega+\alpha_{i}$. Besides, if $g$ is a Riemannian metric on $X$ and if $\varepsilon$ is a positive real number, we can assume by choosing the $\alpha_{j}$ 's small enough that $\left\|\widetilde{J}_{i}-J\right\|_{C^{0}, g}<\varepsilon$ for $1 \leq i \leq N$. Let $m_{1}, \ldots, m_{N}$ be positive integers such that $m_{1}\left(\omega+\alpha_{1}\right), \ldots, m_{N}\left(\omega+\alpha_{N}\right)$ are integral classes. By Theorem 4.5, there exist a positive integer $k$, a family $\left(J_{i}^{\prime}\right)_{1 \leq i \leq N}$ of almost-complex structures on $X$ and a family $\left(C_{i}\right)_{1 \leq i \leq N}$ of two-dimensional submanifolds of $X$ such that for $1 \leq i \leq N, C_{i}$ is $J_{i}^{\prime}$-holomorphic, $\left\|J_{i}^{\prime}-\widetilde{J}_{i}\right\|_{C^{0}, g}<\varepsilon$ and $k m_{i}\left[\omega+\alpha_{i}\right]$ is Poincaré dual to $\left[C_{i}\right]$. Thus, for each integer $i$ between 1 and $N$, $J_{i}^{\prime}$ defines an almost-complex structure on $C_{i}$, which is integrable since $C_{i}$ is twodimensional. Furthermore, $J_{i}^{\prime}$ endows the normal bundle $N_{C_{i}} / X$ with the structure of 
a complex vector bundle over the Riemann surface $\left(C_{i}, J_{i}^{\prime}\right)$. By the Koszul-Malgrange integrability theorem [12, Theorem 2.1.53], there exists a structure of holomorphic line bundle on $N_{C_{i} / X}$. Let $U_{i}$ be a tubular neighbourhood of $C_{i}$ in $X$, diffeomorphic to a neighbourhood of the zero section in $N_{C_{i} / X}$. Pulling back the integrable complex structure on $N_{C_{i} / X}$ by this diffeomorphism, we obtain an integrable complex structure $J_{i}^{\prime \prime}$ on $U_{i}$ whose restriction to $C_{i}$ is equal to $J_{i}^{\prime}$. Since we are free to restrict $U_{i}$, we can assume that $\left\|J_{i}^{\prime \prime}-J_{i}^{\prime}\right\|_{C^{0}, g, U_{i}}<\varepsilon$, so that $\left\|J_{i}^{\prime \prime}-J\right\|_{C^{0}, g, U_{i}}<3 \varepsilon$. If $\varepsilon$ is small enough, this implies that there exist a relatively compact neighbourhood $V_{i}$ of $C_{i}$ in $U_{i}$ as well as a smooth family $\left(J_{t}\right)_{0 \leq t \leq 1}$ of almost-complex structures on $V_{i}$ such that $J_{0}=\left.J\right|_{V_{i}}, J_{1}=\left.J_{i}^{\prime \prime}\right|_{V_{i}}$ and for every $t$ in $[0,1],\left\|J-J_{t}\right\|_{C^{0}, g, V_{i}}<3 \varepsilon$. Let $\chi$ be a smooth real-valued function on $X$ supported in $V_{i}$ such that $\chi \equiv 1$ in a neighbourhood of $C_{i}$. We define an almost-complex structure $J_{i}$ on $X$ by $J_{i}(p)=J_{\chi(p)}(p)$. Then $J_{i}$ is integrable on $V_{i}, C_{i}$ is $J_{i}$-holomorphic and $\left\|J_{i}-J\right\|_{C^{0}}<3 \varepsilon$.

\subsection{Computation of the excess term in the symplectic case}

Our aim in this section is to prove Lehn's formula in full generality for symplectic four-manifolds:

Theorem 4.7 Let $(X, \omega)$ be a symplectic compact four-manifold and $J$ be is an almost-complex structure compatible with $\omega$. If $n$ is a nonnegative integer, the excess contribution $e_{n}$ of Theorem 4.2 is given by

$$
e_{n}=\frac{1}{2} n^{2}(n-1) c_{1}(X) \text {. }
$$

This means that for all integers $n, m$ and for all rational cohomology classes $\alpha$ and $\beta$ on $X$,

$$
\left[\mathfrak{q}_{n}^{\prime}(\alpha), \mathfrak{q}_{m}(\beta)\right]=-n m\left(\mathfrak{q}_{n+m}(\alpha \beta)-\frac{|n|-1}{2} \delta_{n+m, 0}\left(\int_{X} c_{1}(X) \alpha \beta\right) \operatorname{id}_{\mathbb{H}}\right) .
$$

In the integrable case, the statement of the theorem is [25, Proposition 3.15], with slightly different notation. We start by an outline of Lehn's original proof [25, Section 3.4], then we show how to adapt it in the symplectic case. In the sequel, if $Z$ is a triangulable cycle in a topological manifold $Y$, we denote by $[Z]$ the cohomological cycle class of $Z$.

If $X$ is a smooth projective surface and if $C$ is a smooth algebraic curve on $X$, a result of Grojnowski (see Lemma 4.8 below) describes explicitly the class $\left[C^{[n]}\right]$ in $H^{2 n}\left(X^{[n]}, \mathbb{Q}\right)$ in terms of the classes $\mathfrak{q}_{i_{1}}([C]) \ldots \mathfrak{q}_{i_{N}}([C]) .1$, where $i_{1}, \ldots, i_{N}$ are positive integers of total sum $n$. 
Let $X_{0}^{[n]}$ be the set of elements in $X^{[n]}$ whose support is a single point. If $\partial C^{[n]}$ denotes the intersection $C^{[n]} \cap \partial X^{[n]}$, the term

$$
I=\int_{X^{[n]}}\left[X_{0}^{[n]}\right] \cdot\left[\partial C^{[n]}\right]
$$

can be computed in two different ways:

(i) The integral $I$ is equal to $\mathfrak{q}_{-n}(1)\left(\left[\partial C^{[n]}\right]\right)$. Since $C^{[n]}$ and $\partial X^{[n]}$ intersect generically transversally, $\left[\partial C^{[n]}\right]=\left[\partial X^{[n]}\right] \cdot\left[C^{[n]}\right]=-2 c_{1}\left(\mathbb{T}^{n}\right) \cdot\left[C^{[n]}\right]=-2 \mathfrak{d}\left(\left[C^{[n]}\right]\right)$. Thus, $I$ is a linear combination of terms $\mathfrak{q}_{-n}(1) \mathfrak{q}_{i_{1}}([C]) \ldots \mathfrak{q}_{i_{k}}^{\prime}([C]) \ldots \mathfrak{q}_{i_{N}}([C]) .1$, where $i_{1}, \ldots, i_{N}$ are positive integers of total sum $n$. These terms vanish except in two cases:

- $N=1, i_{1}=n$. Then

$$
\mathfrak{q}_{-n}(1) \mathfrak{q}_{n}^{\prime}([C]) .1=-\int_{X} e_{n} \cdot[C] .
$$

- $\quad N=2, i_{1}+i_{2}=n$. Then $\mathfrak{q}_{-n}(1) \mathfrak{q}_{k}([C]) \mathfrak{q}_{n-k}^{\prime}([C]) .1=0$ and

$$
\mathfrak{q}_{-n}(1) \mathfrak{q}_{k}^{\prime}([C]) \mathfrak{q}_{n-k}([C]) .1=-n k \mathfrak{q}_{k-n}([C]) \mathfrak{q}_{n-k}([C]) .1=n k(n-k)[C]^{2} .
$$

This computation gives

$$
I=\frac{1}{n} \int_{X} e_{n} \cdot[C]+\left(\begin{array}{l}
n \\
2
\end{array}\right)[C]^{2} .
$$

(ii) The cycle $C^{[n]}$ intersects transversally $X_{0}^{[n]}$ in its smooth locus. Besides, the intersection $C^{[n]} \cap X_{0}^{[n]}$ is $C_{0}^{[n]}$, which is canonically isomorphic to $C$. Therefore

$$
I=\int_{X^{[n]}}\left[X_{0}^{[n]}\right] \cdot\left[C^{[n]}\right] \cdot\left[\partial X^{[n]}\right]=\operatorname{deg}_{C}\left[\mathcal{O}_{X^{[n]}}\left(\partial X^{[n]}\right)\right]=\operatorname{deg}_{C}\left[\mathcal{O}_{C^{[n]}}\left(\partial C^{[n]}\right)\right],
$$

which is $-n(n-1) \operatorname{deg}_{C} K_{X}$ by direct computation.

The excess terms $e_{n}$ lie in the Neron-Severi group of $X$ so that it is enough to show that for every smooth algebraic curve $C$,

$$
\int_{X}\left[e_{n}-\frac{1}{2} n^{2}(n-1) c_{1}(X)\right] \cdot[C]=0 .
$$

This is proved by comparison of the two expressions obtained for $I$.

Proof of Theorem 4.7 If $\gamma$ is a rational cohomology class on $X$ of even degree, we define the vertex operators $\left(S_{m}(\gamma)\right)_{m \geq 0}$ acting on $\mathbb{H}$ by the formula

$$
\sum_{m \geq 0} S_{m}(\gamma) t^{m}=\exp \left(\sum_{n>0} \frac{(-1)^{n-1}}{n} \mathfrak{q}_{n}(\gamma) t^{n}\right) .
$$


Since $\gamma$ is of even degree, the operators $\left(\mathfrak{q}_{i}(\gamma)\right)_{i>0}$ commute in the usual sense, so that the definition of $S_{m}(\gamma)$ is unambiguous. The following lemma is due to Grojnowski [23] in the integrable case; we refer the reader to Nakajima [35, Section 9.3] for a detailed exposition.

Lemma 4.8 Let $\widetilde{J}$ be an almost-complex structure on $X$ in the deformation class of $J$, and let $C$ be a $\widetilde{J}$-holomorphic curve on $X$. Assume that $\widetilde{J}$ is integrable in a neighbourhood of $C$. Then for any positive integer $n,\left[C^{[n]}\right]=S_{n}([C]) .1$ in $H^{2 n}\left(X^{[n]}, \mathbb{Q}\right)$.

Proof Let $V$ be a small neighbourhood of $C$ in $X$ such that $\widetilde{J}$ is integrable on $V, U$ be a relatively compact neighbourhood of $C$ in $V, m$ and $n$ be two positive integers such that $m>n$ and $J_{n \times(m-n)}^{\text {rel }}$ be a relative integrable complex structure in a neighbourhood $W$ of $Z_{n \times(m-n)}$. If $\widetilde{W}=V \times U^{(n)} \times U^{(m-n)}$, let us assume that $\widetilde{W} \subseteq W$, that $\left.J_{n \times(m-n)}^{\text {rel }}\right|_{\widetilde{W}}=\widetilde{J}$ and that $J_{n \times(m-n)}^{\text {rel }}$ is close to $\widetilde{J}$ in $C^{0}-$ norm. Then $\widetilde{W}_{\text {rel }}^{[m, n]} \cap\left(X^{[m, n]}, J_{n \times(m-n)}^{\text {rel }}\right)$ is exactly the usual incidence variety $U^{[m, n]}$, where $U$ is endowed with the integrable complex structure $\widetilde{J}$. We denote by $\hat{q}_{i}(\alpha)$ the usual Nakajima operators on $U$, in order to distinguish them from the almost-complex Nakajima operators on $X$. Then we have a commutative diagram:

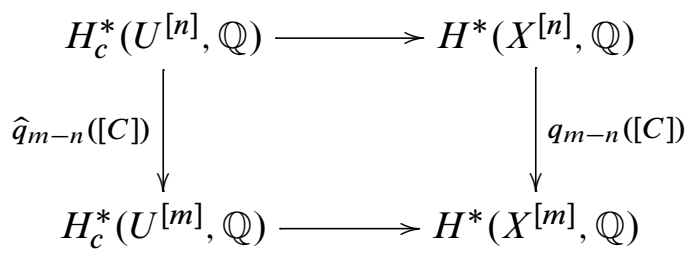

Besides $\widehat{q}_{n}([C]) .1$ lies in $H_{c}^{*}\left(U^{[n]}, \mathbb{Q}\right)$ and its image in $H^{*}\left(X^{[n]}, \mathbb{Q}\right)$ is $q_{n}([C]) .1$. Since the identity of the lemma holds in $H_{c}^{2 n}\left(U^{[n]}, \mathbb{Q}\right)$ for the classical Nakajima operators, we obtain the result.

If $(C, \widetilde{J})$ satisfies the hypotheses of Lemma 4.8, Lehn's computations recalled above apply verbatim and give

$$
\int_{X}\left[e_{n}-\frac{1}{2} n^{2}(n-1) c_{1}(X)\right] \cdot[C]=0 .
$$

By Proposition $4.6, H^{2}(X, \mathbb{Q})$ is spanned by cohomology classes of such holomorphic curves. Since the intersection form of $X$ is nondegenerate on $H^{2}(X, \mathbb{Q})$, we get $e_{n}=\frac{1}{2} n^{2}(n-1) c_{1}(X)$. 
The derivative of the Nakajima operators can be explicitly expressed using the Virasoro operators $\mathfrak{L}_{n}(\alpha)$ defined in [25, Section 3.1]:

Corollary 4.9 If $(X, \omega)$ is a symplectic compact four-manifold and if $J$ is a compatible almost-complex structure, then for every $n$ in $\mathbb{Z}$,

$$
\mathfrak{q}_{n}^{\prime}(\alpha)=n \mathfrak{L}_{n}(\alpha)-\frac{1}{2} n(|n|-1) \mathfrak{q}_{n}\left(c_{1}(X) \alpha\right) .
$$

For the proof, see [25, page 180].

\section{The ring structure of $H^{*}\left(X^{[n]}, \mathbb{Q}\right)$}

\subsection{Geometric tautological Chern characters}

Let $(X, J)$ be an almost-complex compact four-manifold, $n$ be a positive integer and $\lambda, v$ be the homotopy classes in $\left[X^{[n+1, n]}: X^{[n]}\right]$ and $\left[X^{[n+1, n]}: X^{[n+1]}\right]$ introduced in Section 2.4.

If $E$ is a complex vector bundle on $X$, it is possible to associate with $E$ a sequence of tautological vector bundles $\left(E^{[n]}\right)_{n>0}$ on $X^{[n]}$. These tautological bundles are constructed in [21, Section 5] using relative holomorphic structures on $E$, and their classes in complex $K$-theory are shown to be independent of these auxiliary structures [21, Proposition 5.3]. This construction yields tautological morphisms from $K(X)$ to $K\left(X^{[n]}\right)$.

If $F$ is the class in $K\left(X^{[n+1, n]}\right)$ defined at the beginning of Section 4.1, then the tautological bundles $E^{[n]}$ and $E^{[n+1]}$ are related through the identity $v^{*} E^{[n+1]}=$ $\lambda^{*} E^{[n]}+\rho^{*} E \otimes F$ in $K\left(X^{[n+1, n]}\right)$, which is a $K$-theoretical analog of (2-4) (see $\left[21\right.$, Proposition 5.5]). This gives in $H^{\text {even }}\left(X^{[n+1, n]}, \mathbb{Q}\right)$ the relation

$$
\nu^{*}\left(\operatorname{ch}\left(E^{[n+1]}\right)\right)=\lambda^{*}\left(\operatorname{ch}\left(E^{[n]}\right)\right)+\rho^{*} \operatorname{ch}(E) \cdot \operatorname{ch}(F) .
$$

Lemma 5.1 For every class $\alpha$ in $H^{\text {even }}(X, \mathbb{Q})$ and every positive integer $n$, there exists a unique class $G(\alpha, n)$ in $H^{\text {even }}\left(X^{[n]}, \mathbb{Q}\right)$ such that $G(\alpha, 1)=\alpha$ and for every positive integer $n$,

$$
\nu^{*} G(\alpha, n+1)-\lambda^{*} G(\alpha, n)=\rho^{*} \alpha \cdot \operatorname{ch}(F) .
$$


Proof The Chern character on $X$ gives an isomorphism between $K(X) \otimes_{\mathbb{Z}} \mathbb{Q}$ and $H^{\text {even }}(X, \mathbb{Q})$. Therefore, we can define the classes $G(\alpha, n)$ in $H^{\text {even }}\left(X^{[n]}, \mathbb{Q}\right)$ as follows: if $y$ is the unique class in $K(X) \otimes_{\mathbb{Z}} \mathbb{Q}$ such that $\operatorname{ch}(y)=\alpha$, then $G(\alpha, n)=$ $\operatorname{ch}\left(y^{[n]}\right)$. Furthermore, $G(\alpha, n)$ is unique since $v_{*} \nu^{*}=(n+1)$ id.

\subsection{Virtual tautological Chern characters}

In this section, we extend Lemma 5.1 to odd cohomology classes. We adapt the method originally developed in the projective case by Li, Qin and Wang in [29, Section 5].

Proposition 5.2 For every class $\alpha$ in $H^{*}(X, \mathbb{Q})$ and every $n$ in $\mathbb{N}^{*}$, there exists a unique class $G(\alpha, n)$ in $H^{*}\left(X^{[n]}, \mathbb{Q}\right)$ such that $G(\alpha, 1)=\alpha$ and for every positive integer $n$,

$$
\nu^{*} G(\alpha, n+1)-\lambda^{*} G(\alpha, n)=\rho^{*} \alpha \cdot \operatorname{ch}(F) .
$$

Remark 5.3 If $X$ is a projective surface, if $Y_{n}$ is the incidence locus in $X^{[n]} \times X$ and if $\operatorname{td}(X)$ is the Todd class of $X$, then $G(\alpha, n)=\operatorname{pr}_{1}^{*}\left[\operatorname{ch}\left(\mathcal{O}_{Y_{n}}\right) \cdot \operatorname{pr}_{2}^{*} \alpha \cdot \operatorname{pr}_{2}^{*} \operatorname{td}(X)\right]$ (see [29, Lemma 5.8]).

Proof We adopt the notation of Section 3.5, especially those of Notation 3.24.

- The projection from $W\left(W^{\prime}, W^{\prime \prime}\right)$ to $X$ is denoted by $t\left(t^{\prime}, t^{\prime \prime}\right.$, resp.).

- The projection from

$$
\begin{gathered}
W_{\mathrm{rel}}^{\prime[n]} \times_{X^{(n)}} W^{\prime}, \quad W_{\mathrm{rel}}^{\prime \prime[n+1]} \times_{X^{(n+1)}} W^{\prime \prime}, \quad W_{\mathrm{rel}}^{[n]} \times_{X^{(n)} \times X} W, \\
W_{\mathrm{rel}}^{[n+1]} \times_{X^{(n)} \times X} W, \quad W_{\mathrm{rel}}^{[n+1, n]} \times_{X^{(n)} \times X} W
\end{gathered}
$$

to

$$
W^{\prime}, \quad W^{\prime \prime}, \quad W, \quad W, \quad W, \quad \text { resp. }
$$

is denoted by $\pi_{1}, \pi_{2}, \pi_{3}, \pi_{4}, \pi_{5}$, resp.

- The first and second projections from $W \times_{X^{(n)} \times X} W$ to $W$ are denoted by $\pi_{6}$ and $\pi_{7}$.

Thanks to Proposition 3.19, we can assume that $\mathcal{O}_{n}^{\infty}, \mathcal{O}_{n+1}^{\infty}, \widetilde{\mathcal{O}}_{n}^{\infty}$ and $\widetilde{\mathcal{O}}_{n+1}^{\infty}$ admit global smooth locally free resolutions. Let $\mu_{n}$ (resp. $\mu_{n+1}, \tilde{\mu}_{n}, \widetilde{\mu}_{n+1}$ ) be the Chern character of the relative incidence sheaf $\mathcal{O}_{n}\left(\operatorname{resp} . \mathcal{O}_{n+1}, \widetilde{\mathcal{O}}_{n}, \widetilde{\mathcal{O}}_{n+1}\right)$ in

$$
\begin{aligned}
H_{\mathfrak{Y}} n & \left(W_{\mathrm{rel}}^{\prime[n]} \times_{X^{(n)}} W^{\prime}\right) \quad\left(\text { resp. } H_{\mathfrak{Y})}^{*}\left(W_{\mathrm{rel}}^{\prime \prime}{ }^{[n+1]} \times_{X^{(n+1)}} W^{\prime \prime}\right),\right. \\
& \left.H_{\tilde{\mathfrak{Y}}_{n}}^{*}\left(W_{\mathrm{rel}}^{[n]} \times_{X^{(n)} \times X} W\right), H_{\tilde{\mathfrak{Y}}_{n+1}^{*}}^{*}\left(W_{\mathrm{rel}}^{[n+1]} \times_{X^{(n)} \times X} W\right)\right) .
\end{aligned}
$$


Since $\bar{p}$ (resp. $\bar{q}, p, q$ ) is finite on $\mathfrak{Y}_{n}\left(\right.$ resp. $\mathfrak{Y}_{n+1}, \tilde{\mathfrak{Y}}_{n}, \tilde{\mathfrak{Y}}_{n+1}$ ), we can define six cohomology classes as follows:

$$
\begin{aligned}
& K(\alpha, n)=\bar{p}_{*}\left[\mu_{n} \cdot \pi_{1}^{*} t^{\prime *}(\alpha \cdot \operatorname{td}(X))\right] \text { in } H^{*}\left(W_{\mathrm{rel}}^{\prime[n]}, \mathbb{Q}\right), \\
& K(\alpha, n+1)=\bar{q}_{*}\left[\mu_{n+1} \cdot \pi_{2}^{*} t^{\prime \prime *}(\alpha \cdot \operatorname{td}(X))\right] \text { in } H^{*}\left(W_{\mathrm{rel}}^{\prime \prime[}[n+1], \mathbb{Q}\right) \text {, } \\
& \widetilde{K}(\alpha, n)=p_{*}\left[\tilde{\mu}_{n} \cdot \pi_{3}^{*} t^{*}(\alpha \cdot \operatorname{td}(X))\right] \text { in } H^{*}\left(W_{\mathrm{rel}}^{[n]}, \mathbb{Q}\right), \\
& \tilde{K}(\alpha, n+1)=q_{*}\left[\tilde{\mu}_{n+1} \cdot \pi_{4}^{*} t^{*}(\alpha \cdot \operatorname{td}(X))\right] \text { in } H^{*}\left(W_{\mathrm{rel}}^{[n+1]}, \mathbb{Q}\right) \text {, } \\
& G(\alpha, n)=\left.K(\alpha, n)\right|_{X^{[n]}} \text { in } H^{*}\left(X^{[n]}, \mathbb{Q}\right), \\
& G(\alpha, n+1)=\left.K(\alpha, n+1)\right|_{X^{[n+1]}} \text { in } H^{*}\left(X^{[n+1]}, \mathbb{Q}\right) .
\end{aligned}
$$

Then, by Proposition 3.10 (ii),

$$
\begin{aligned}
\psi^{*} \tilde{K}(\alpha, n+1)-\phi^{*} \tilde{K}(\alpha, n) & =\tilde{p}_{*}\left[\left(\psi_{W}^{*} \tilde{\mu}_{n+1}-\phi_{W}^{*} \tilde{\mu}_{n}\right) \cdot \pi_{5}^{*} t^{*}(\alpha \cdot \operatorname{td}(X))\right] \\
& =\tilde{p}_{*}\left[\tilde{p}^{*} \operatorname{ch}(\mathcal{L}) \cdot \bar{\rho}_{W}^{*} \operatorname{ch}\left(\left[\mathcal{O}_{\Delta}^{\infty}\right]\right) \cdot \bar{\rho}_{W}^{*} \pi_{7}^{*} t^{*}(\alpha \cdot \operatorname{td}(X))\right] .
\end{aligned}
$$

Since $\operatorname{ch}\left(\left[\mathcal{O}_{\Delta}^{\infty}\right]\right)$ is supported in $\Delta_{\text {rel }}$, for every $\beta$ in $H^{*}\left(W \times_{X^{(n)} \times X} W, \mathbb{Q}\right)$, we obtain that $\operatorname{ch}\left(\left[\mathcal{O}_{\Delta}^{\infty}\right]\right) \cdot \pi_{6}^{*} \beta=\operatorname{ch}\left(\left[\mathcal{O}_{\Delta}^{\infty}\right]\right) \cdot \pi_{7}^{*} \beta$. Using the diagram

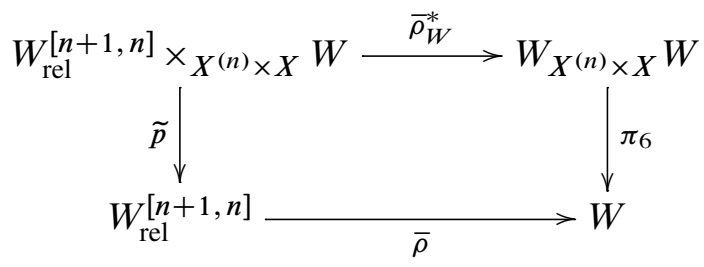

we get

$$
\begin{aligned}
\psi^{*} \tilde{K}(\alpha, n+1)-\phi^{*} \tilde{K}(\alpha, n) & =\operatorname{ch}(\mathcal{L}) \tilde{p}_{*} \bar{\rho}_{W}^{*}\left[\operatorname{ch}\left(\left[\mathcal{O}_{\Delta}^{\infty}\right]\right) \cdot \pi_{6}^{*} t^{*}(\alpha \cdot \operatorname{td}(X))\right] \\
& =\operatorname{ch}(\mathcal{L}) \bar{\rho}^{*}\left(\pi_{6 *} \operatorname{ch}\left(\left[\mathcal{O}_{\Delta}^{\infty}\right]\right) \cdot t^{*}(\alpha \cdot \operatorname{td}(X))\right) .
\end{aligned}
$$

Lemma 5.4 (i) If $\tilde{t}=t \times_{X^{(n)} \times X} t: W_{X^{(n)} \times X} W \longrightarrow X \times X$ and if $i: X \rightarrow X \times X$ is the diagonal injection, then $\operatorname{ch}\left(\left[\mathcal{O}_{\Delta}^{\infty}\right]\right)=\widetilde{t}^{*} i_{*} \operatorname{td}(X)^{-1}$.

(ii) $\lambda^{*} G(\alpha, n)=\left.\phi^{*} \widetilde{K}(\alpha, n)\right|_{X^{[n+1, n]}}$.

(iii) $\nu^{*} G(\alpha, n+1)=\left.\psi^{*} \widetilde{K}(\alpha, n+1)\right|_{X^{[n+1, n]}}$.

Proof (i) The class $\left[\mathcal{O}_{\Delta}^{\infty}\right]$ in $K_{\Delta_{\text {rel }}}\left(W_{X^{(n)} \times X} W\right)$ is the pullback by the map $\tilde{t}$ of the class $\left[\mathcal{C}_{\Delta_{X}}^{\infty}\right]$ in $K_{\Delta_{X}}(X \times X)$. Besides, the differentiable Grothendieck-Riemann-Roch theorem for immersions [3, Theorem 3.3] yields $\operatorname{ch}\left(\left[\mathcal{C}_{\Delta_{X}}^{\infty}\right]\right)=i_{*} \operatorname{td}(X)^{-1}$. 
(ii) If $\left(W, J_{n \times 1}^{\mathrm{rel}}\right)$ and $\left(W^{\prime}, J_{n}^{\text {rel }}\right)$ satisfy the compatibility condition (A) of Section 2.4, then we have $\lambda^{*} G(\alpha, n)=\left.\phi^{*} \widetilde{K}(\alpha, n)\right|_{X^{[n+1, n]}}$. To conclude in the general case, we argue exactly as in Lemma 3.26.

(iii) The proof is the same as (ii), replacing condition (A) by condition (B).

By (i), we get $\pi_{6 *} \operatorname{ch}\left(\left[\mathcal{O}_{\Delta}^{\infty}\right]\right)=\pi_{6 *} \widetilde{t}^{*} i_{*} \operatorname{td}(X)^{-1}=t^{*} \operatorname{pr}_{1 *} i_{*} \operatorname{td}(X)^{-1}=t^{*} \operatorname{td}(X)^{-1}$, so that $\psi^{*} \widetilde{K}(\alpha, n+1)-\phi^{*} \widetilde{K}(\alpha, n)=\operatorname{ch}(\mathcal{L}) \bar{\rho}^{*} t^{*} \alpha$. By points (ii) and (iii), we obtain the relation $\lambda^{*} G(\alpha, n)-v^{*} G(\alpha, n+1)=\operatorname{ch}(F) \cdot \rho^{*} \alpha$. This finishes the proof of Proposition 5.2.

\subsection{The ring structure and the crepant resolution conjecture}

In this section, $X$ is a symplectic compact four-manifold endowed with a compatible almost-complex structure.

We introduce operators acting on $\mathbb{H}=\bigoplus_{n \in \mathbb{N}} H^{*}\left(X^{[n]}, \mathbb{Q}\right)$ by cup product with the components of the virtual tautological Chern characters constructed in Section 5.2.

If $\alpha$ is a homogeneous rational cohomology class on $X$ and if $i, n$ are positive integers, we denote by $G_{i}(\alpha, n)$ the $(|\alpha|+2 i)$-th component of $G(\alpha, n)$ and by $\mathfrak{S}_{i}(\alpha)$ the operator on $\mathbb{H}$ that acts by cup product with $G_{i}(\alpha, n)$ on $H^{*}\left(X^{[n]}, \mathbb{Q}\right)$.

We now state a result, originally proved by Lehn for geometric tautological Chern characters [25, Theorem 4.2] and generalized by Li, Qin and Wang for virtual ones [29, Lemma 5.8]. We include a proof for the sake of completeness.

Proposition 5.5 For all homogeneous rational cohomology classes $\alpha, \beta$ on $X$ and for any positive integer $k,\left[\mathfrak{S}_{k}(\alpha), \mathfrak{q}_{1}(\beta)\right]=(1 / k !) \mathfrak{q}_{1}^{(k)}(\alpha \beta)$.

Proof Let $\alpha, \beta$ be homogeneous rational cohomology classes on $X$ and $n$ be a positive integer. The operator $\mathfrak{q}_{1}(\beta): H^{*}\left(X^{[n]}, \mathbb{Q}\right) \longrightarrow H^{*}\left(X^{[n+1]}, \mathbb{Q}\right)$ is given by $\mathfrak{q}_{1}(\beta), \tau=v_{*}\left(\lambda^{*} \tau \cdot \rho^{*} \beta\right)$. Therefore

$$
\begin{aligned}
G(\alpha, n+1) \cdot\left(\mathfrak{q}_{1}(\beta) . \tau\right) & -\mathfrak{q}_{1}(\beta) \cdot(G(\alpha, n) \cdot \tau) \\
= & v_{*}\left(\lambda^{*} \tau \cdot v^{*} G(\alpha, n+1) \cdot \rho^{*} \beta\right)-v_{*}\left(\lambda^{*}(\tau \cdot G(\alpha, n)) \cdot \rho^{*} \beta\right) \\
= & v_{*}\left(\operatorname{ch}(F) \cdot \lambda^{*} \tau \cdot \rho^{*}(\alpha \beta)\right) \\
= & \sum_{k \geq 0} \frac{1}{k !} v_{*}\left(c_{1}(F)^{k} \lambda^{*} \tau \cdot \rho^{*}(\alpha \beta)\right) .
\end{aligned}
$$


On the other hand, since $\mathfrak{q}_{1}(\alpha \beta)$ is given on $H^{*}\left(X^{[n]}, \mathbb{Q}\right)$ by the action by correspondence of the Poincaré dual of $\rho^{*}(\alpha \beta)$, we have by Lemma 4.3

$$
\begin{aligned}
\mathfrak{q}_{1}^{(k)}(\alpha \beta) & =\left[\left(\operatorname{PD}\left(\rho^{*}(\alpha \beta)\right)\right)_{*}\right]^{(k)}=\left[\left(\operatorname{PD}\left(\rho^{*}(\alpha \beta)\right) \cap c_{1}(F)\right)_{*}\right]^{(k-1)} \\
& =\cdots=\left[\operatorname{PD}\left(\rho^{*}(\alpha \beta)\right) \cap c_{1}(F)^{k}\right]_{*} .
\end{aligned}
$$

This yields the result.

As explained in [25, Remark 4.5], Proposition 5.5, Theorem 4.7 and Corollary 4.4 yield a complete description of the operators $\mathfrak{S}_{k}(\alpha)$.

The forthcoming Theorems 5.6, 5.7 and 5.9 extend to Hilbert schemes of symplectic manifolds the analogous results for projective surfaces of Li, Qin and Wang [29, Theorem 5.2; 30, Theorem 4.1] and, for Theorem 5.9, of Lehn and Sorger [27, Theorem 1.1] and Qin and Wang [37, Theorem 5.13]. The first two results are formal consequences of the various relations between $\mathfrak{q}_{n}(\alpha), \mathfrak{d}, \mathfrak{L}_{n}(\alpha)$ and $\mathfrak{S}_{i}(\alpha)$ listed in [30, Theorem 2.1]. Thus, the two following theorems are formal consequences of Theorem 4.7, Corollary 4.9 and Proposition 5.5:

Theorem 5.6 If $0 \leq i<n$ and if $\alpha$ runs through a fixed basis of $H^{*}(X, \mathbb{Q})$, the classes $G_{i}(\alpha, n)$ generate the ring $H^{*}\left(X^{[n]}, \mathbb{Q}\right)$.

Theorem 5.7 For every integer $n$, the ring $H^{*}\left(X^{[n]}, \mathbb{Q}\right)$ can be built by universal formulae from the ring $H^{*}(X, \mathbb{Q})$ and the first Chern class of $X$ in $H^{2}(X, \mathbb{Q})$.

In the case where $b_{1}(X)$ vanishes, Theorem 5.6 implies that the rings $H^{*}\left(X^{[n]}, \mathbb{Q}\right)$ are generated by the components of the tautological Chern characters $\operatorname{ch}\left(E^{[n]}\right)$, where $E$ runs through all complex vector bundles on $X$.

We now turn to the study of a particular case of Ruan's crepant resolution conjecture. Orbifold cohomology provides a geometrical approach to the rings $H^{*}\left(X^{[n]}, \mathbb{Q}\right)$. If $J$ is an adapted almost-complex structure on $X$, the symmetric product $X^{(n)}$ is an almost-complex Gorenstein orbifold. The orbifold cohomology ring $H_{\text {orb }}^{*}\left(X^{(n)}, \mathbb{Q}\right)$ is $\mathbb{Z}$-graded and depends only on the deformation class of $J$ (see $[9 ; 1 ; 15]$ ).

After works by Lehn and Sorger [26; 27], Li, Qin and Wang [28; 29; 30], Fantechi and Göttsche [15] and Uribe [42], Qin and Wang [37, Section 2.6] developed a set of axioms that characterize $H_{\mathrm{orb}}^{*}\left(X^{(n)}, \mathbb{Q}\right)$ as a ring. Here is their result, as stated in $[1$, Theorem 5.24]: 
Theorem 5.8 Let $A$ be a graded unitary ring, $(X, J)$ be an almost-complex compact four-manifold and $\mathcal{H}\left(H^{*}(X, \mathbb{C})\right)$ be the Heisenberg superalgebra of $H^{*}(X, \mathbb{C})$. We assume that:

(i) The ring $A$ is an irreducible $\mathcal{H}\left(H^{*}(X, \mathbb{C})\right)$-module and 1 is a highest weight vector.

(ii) For any $\alpha$ in $H^{*}(X, \mathbb{C})$ and for any nonnegative integer $i$, there exist classes $O_{i}(\alpha, n)$ in $A^{|\alpha|+2 i}$ such that if $\mathfrak{D}_{i}(\alpha)$ is the left multiplication by $\bigoplus_{n} O_{i}(\alpha, n)$ on $A$ and if $\mathfrak{d}=\mathfrak{D}_{1}(1)$, then:

- For all $\alpha, \beta$ in $H^{*}(X, \mathbb{C})$, for every nonnegative integer $k$,

$$
\left[\mathfrak{D}_{k}(\alpha), \mathfrak{q}_{1}(\beta)\right]=\mathfrak{q}_{1}^{(k)}(\alpha \beta) .
$$

- If $\delta_{X}$ is the class in $H^{*}(X, \mathbb{C})^{\otimes 3}$ mapped by the Künneth isomorphism to the cycle class of the diagonal in $X^{3}$, then

$$
\sum_{l_{1}+l_{2}+l_{3}=0}: \mathfrak{q}_{l_{1}} \mathfrak{q}_{l_{2}} \mathfrak{q}_{3}:\left(\delta_{X}\right)=-6 \mathfrak{d} .
$$

Then the rings $A$ and $H_{\text {orb }}^{*}\left(X^{(n)}, \mathbb{C}\right)$ are isomorphic.

(We use the physicists' normal ordering convention

$: \mathfrak{q}_{l_{1}} \mathfrak{q}_{l_{2}} \mathfrak{q}_{l_{3}}:=\mathfrak{q}_{m_{1}} \mathfrak{q}_{m_{2}} \mathfrak{q}_{m_{3}}$, where $\left\{l_{1}, l_{2}, l_{3}\right\}=\left\{m_{1}, m_{2}, m_{3}\right\}$ and $\left.m_{1} \leq m_{2} \leq m_{3}\right)$.

We apply this theorem to prove Ruan's conjecture for the symmetric products of a symplectic four-manifold with torsion first Chern class.

Theorem 5.9 Let $(X, \omega)$ be a symplectic compact four-manifold with vanishing first Chern class in $H^{2}(X, \mathbb{Q})$. Then, for every positive integer $n$, Ruan's crepant conjecture holds for $X^{(n)}$, ie the rings $H^{*}\left(X^{[n]}, \mathbb{C}\right)$ and $H_{\mathrm{orb}}^{*}\left(X^{(n)}, \mathbb{C}\right)$ are isomorphic.

Proof Let $O_{k}(\alpha, n)=k ! \mathfrak{S}_{k}(\alpha, n)$. The first condition of Theorem 5.8 (ii) is exactly Proposition 5.5. The second condition is a formal consequence of the Nakajima relations and of the formulae $\left[\mathfrak{q}_{n}^{\prime}(\alpha), \mathfrak{q}_{m}(\beta)\right]=-n m \mathfrak{q}_{n+m}(\alpha \beta), \mathfrak{q}_{n}^{\prime}(\alpha)=n \mathfrak{L}_{n}(\alpha)$.

\section{The cobordism class of $X^{[n]}$}

In this section, $(X, J)$ is an almost-complex compact four-manifold, and no symplectic hypotheses are required. The almost-complex Hilbert schemes $X^{[n]}$ are endowed with a stable almost complex structure, hence define almost-complex cobordism classes. By 
classical results of Novikov [36] and Milnor [33], the almost-complex cobordism class of $X^{[n]}$ is completely determined by the Chern numbers

$$
\int_{X^{[n]}} P\left[c_{1}\left(X^{[n]}\right), \ldots, c_{2 n}\left(X^{[n]}\right)\right],
$$

where $P$ runs through all polynomials $P$ in $\mathbb{Q}\left[T_{1}, \ldots, T_{2 n}\right]$ of weighted degree $4 n$, each variable $T_{k}$ having degree $2 k$. We intend to prove the following result:

Theorem 6.1 The almost-complex cobordism class of $X^{[n]}$ depends only on the almost-complex cobordism class of $X$.

This means that if $P$ is a weighted polynomial in $\mathbb{Q}\left[T_{1}, \ldots, T_{2 n}\right]$ of degree $4 n$, there exists a weighted polynomial $\widetilde{P}\left[T_{1}, T_{2}\right]$ of degree 4 , depending only on $P$ and $n$, such that

$$
\int_{X^{[n]}} P\left[c_{1}\left(X^{[n]}\right), \ldots, c_{2 n}\left(X^{[n]}\right)\right]=\int_{X} \tilde{P}\left[c_{1}(X), c_{2}(X)\right] .
$$

This result has been proved by Ellinsgrud, Göttsche and Lehn [14, Theorem 0.1] when $X$ is projective. In Sections 6.1, 6.2 and 6.3, we adapt the authors' original proof in a relative setting. Throughout this section, we use extensively the notation of Section 3.5, especially Notation 3.24.

\subsection{Computation of $T X^{[n]}$ in $K$-theory}

Let $J_{n \times 1}^{\text {rel }}\left(\right.$ resp. $\left.J_{n}^{\text {rel }}, J_{n+1}^{\text {rel }}\right)$ be a relative integrable complex structure in a neighbourhood $W$ (resp. $W^{\prime}, W^{\prime \prime}$ ) of $Z_{n \times 1}$ (resp. $Z_{n}, Z_{n+1}$ ). We denote the class of

$$
T^{\text {rel }} W_{\text {rel }}^{[n]} \quad\left(\text { resp. } T^{\text {rel }} W_{\text {rel }}^{[n+1]}, \quad T^{\text {rel }} W_{\text {rel }}^{\prime[n]}, \quad T^{\text {rel }} W_{\text {rel }}^{\prime \prime}[n+1]\right. \text { ) }
$$

in $K^{\mathrm{rel}}\left(W_{\mathrm{rel}}^{[n]}\right)\left(\operatorname{resp} . K^{\mathrm{rel}}\left(W_{\mathrm{rel}}^{[n+1]}\right), K^{\mathrm{rel}}\left(W_{\mathrm{rel}}^{\prime[n]}\right), K^{\mathrm{rel}}\left(W_{\text {rel }}^{\prime \prime[n+1]}\right)\right)$ by $\widetilde{\kappa}_{n}\left(\operatorname{resp} . \widetilde{\kappa}_{n+1}\right.$, $\left.\kappa_{n}, \kappa_{n+1}\right)$.

Lemma 6.2 The restriction to $X^{[n]}$ (resp. $X^{[n+1]}$ ) of the topological class of $\kappa_{n}$ (resp. $\left.\kappa_{n+1}\right)$ is the class of the complex vector bundle $T X^{[n]}$ (resp. $\left.T X^{[n+1]}\right)$ in $K\left(X^{[n]}\right)\left(\right.$ resp. $\left.K\left(X^{[n+1]}\right)\right)$ given by the stable almost-complex structure on $X^{[n]}$ (resp. $\left.X^{[n+1]}\right)$.

Proof If $J_{n}^{\text {rel }}$ satisfies the conditions ( $\left.\mathscr{C}\right)$ listed in [43, page 711], then $X_{J_{n}^{\text {rel }}}^{[n]}$ is smooth. Besides, the construction performed in [43] of the stable almost-complex structure of $X_{\boldsymbol{J}_{n}^{\text {rel }}}^{[n}$ shows that $T X^{[n]}$ and $\left.T^{\mathrm{rel}} W_{\mathrm{rel}}^{[n]}\right|_{X^{[n]}}$ have the same class in $K\left(X^{[n]}\right)$. Since relative almost-complex structures satisfying the conditions (C) can be chosen arbitrary close to $J$ in $C^{0}$-norm, Proposition 2.13 implies, after shrinking $W$ if necessary, 
that $J_{n}^{\text {rel }}$ can be joined by a smooth path $\left\{J_{n, t}^{\text {rel }}\right\}_{t \in[0,1]}$ to another relative integrable structure satisfying the conditions $(\mathscr{C})$. By rigidity of the topological $K$-theory, the class of $\left.T^{\mathrm{rel}}\left[W_{\mathrm{rel}}^{[n]}, J_{n, t}^{\mathrm{rel}}\right]\right|_{X^{[n]}}$ in $K\left(X^{[n]}\right)$ is independent of $t$. The result follows.

Remark 6.3 For an arbitrary $J_{n}^{\text {rel }}$, by Theorem 2.18 (ii), $X_{J_{n}^{\text {rel }}}^{[n]}$ is only a topological manifold. Therefore, the advantage of using $T^{\mathrm{rel}} W_{\mathrm{rel}}^{[n]}$ is that this complex vector bundle is defined for any relative integrable complex structure $J_{n}^{\text {rel }}$.

Proposition 6.4 The following identities hold in $K^{\mathrm{rel}}\left(W_{\mathrm{rel}}^{[n]}\right)$ and $K^{\mathrm{rel}}\left(W_{\mathrm{rel}}^{[n+1]}\right)$ respectively:

$$
\widetilde{\kappa}_{n}=p_{*}\left(\widetilde{\mathcal{O}}_{n}+\widetilde{\mathcal{O}}_{n}^{\vee}-\widetilde{\mathcal{O}}_{n} \cdot \tilde{\mathcal{O}}_{n}^{\vee}\right), \quad \widetilde{\kappa}_{n+1}=q_{*}\left(\widetilde{\mathcal{O}}_{n+1}+\widetilde{\mathcal{O}}_{n+1}^{\vee}-\widetilde{\mathcal{O}}_{n+1} \cdot \tilde{\mathcal{O}}_{n+1}^{\vee}\right) .
$$

Proof Let $\phi: U \stackrel{\sim}{\longrightarrow} Z \times V$ be a relative holomorphic chart on $W^{\prime}, \mathfrak{p}$ be the first projection from $Z^{[n]} \times Z$ to $Z^{[n]}$ and $Y_{n}$ be the incidence locus in $Z^{[n]} \times Z$ defined by (2-1). Since $p$ is finite on $\tilde{\mathfrak{Y}}_{n}$, we obtain by Proposition 3.13:

$$
\begin{aligned}
& \left.p_{*} \mathcal{H o m}_{\mathcal{O}_{\text {rel }}}\left(\tilde{\mathcal{J}}_{n}, \widetilde{\mathcal{O}}_{n}\right)\right|_{U}
\end{aligned}
$$

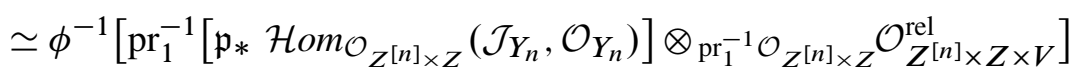

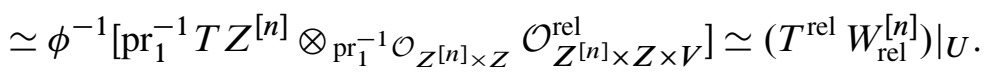

These local isomorphisms patch together into a global isomorphism between the two relative holomorphic bundles $p_{*} \mathcal{H o m}_{\mathcal{O}_{\text {rel }}}\left(\widetilde{\mathcal{J}}_{n}, \widetilde{\mathcal{O}}_{n}\right)$ and $T W_{\text {rel }}^{[n]}$. If $i$ is a nonnegative integer, Proposition 3.6 yields the isomorphism

$$
\begin{aligned}
& \left.\mathcal{E} x t_{\mathcal{O}^{\text {rel }}}^{i}\left(\widetilde{\mathcal{O}}_{n}, \mathcal{O}^{\text {rel }}\right)\right|_{U} \\
& \simeq \phi^{-1}\left(\operatorname{pr}_{1}^{-1} \mathcal{E} x t_{\mathcal{O}_{Z}^{[n]} \times Z}^{i}\left(\mathcal{O}_{Y_{n}}, \mathcal{O}_{Z^{[n]} \times Z}\right) \otimes_{\operatorname{pr}_{1}^{-1} \mathcal{O}_{Z^{[n]_{\times}}}} \mathcal{O}_{Z^{[n]} \times Z \times V}^{\mathrm{rel}}\right) .
\end{aligned}
$$

Since $Y_{n}$ has codimension 2 in $Z^{[n]} \times Z, \mathcal{E} x t_{\mathcal{O}^{\text {rel }}}^{i}\left(\widetilde{\mathcal{O}}_{n}, \mathcal{O}^{\text {rel }}\right)=0$ for $i<2$ by [13, Proposition 18.4, Theorem 18.7]. Besides, $\widetilde{\mathcal{J}}_{n}$ locally admits a free resolution of length 2 . Using Proposition 3.16 (i), we get the following equalities in $K_{\widetilde{\mathfrak{Y}}_{n}}^{\text {rel }}\left(W_{\text {rel }}^{[n]} \times_{X}{ }^{(n)} \times X W\right)$ :

$$
\begin{aligned}
& \tilde{\mathcal{J}}_{n}^{\vee} . \widetilde{\mathcal{O}}_{n}=\mathcal{H o m}_{\mathcal{O}^{\text {rel }}}\left(\tilde{\mathcal{J}}_{n}, \widetilde{\mathcal{O}}_{n}\right)-\mathcal{E} x t_{\mathcal{O}^{\text {rel }}}^{1}\left(\tilde{\mathcal{J}}_{n}, \widetilde{\mathcal{O}}_{n}\right)+\mathcal{E} x t_{\mathcal{O}^{\text {rel }}}^{2}\left(\tilde{\mathcal{J}}_{n}, \widetilde{\mathcal{O}}_{n}\right) \\
& =\mathcal{H o m}_{\mathcal{O}^{\text {rel }}}\left(\tilde{\mathcal{J}}_{n}, \widetilde{\mathcal{O}}_{n}\right)-\mathcal{E} x t_{\mathcal{O}^{\text {rel }}}^{2}\left(\widetilde{\mathcal{O}}_{n}, \widetilde{\mathcal{O}}_{n}\right) \\
& =\mathcal{H o m}_{\mathcal{O}^{\text {rel }}}\left(\tilde{\mathcal{J}}_{n}, \widetilde{\mathcal{O}}_{n}\right)-\mathcal{E} x t_{\mathcal{O}^{\text {rel }}}^{2}\left(\widetilde{\mathcal{O}}_{n}, \mathcal{O}^{\text {rel }}\right) \\
& =\mathcal{H o m}_{\mathcal{O}_{\text {rel }}}\left(\tilde{\mathcal{J}}_{n}, \widetilde{\mathcal{O}}_{n}\right)-\widetilde{\mathcal{O}}_{n}^{\vee}
\end{aligned}
$$

so that $p_{*} \mathcal{H}_{o m_{\mathcal{O}^{\text {rel }}}}\left(\tilde{\mathcal{J}}_{n}, \widetilde{\mathcal{O}}_{n}\right)=p_{*}\left[\left(\mathcal{O}^{\text {rel }}-\widetilde{\mathcal{O}}_{n}^{\vee}\right) \cdot \widetilde{\mathcal{O}}_{n}+\widetilde{\mathcal{O}}_{n}^{\vee}\right]$. The proof of the second identity is exactly the same. 


\subsection{Comparison of $T X^{[n]}$ and $T X^{[n+1]}$ via the incidence variety $X^{[n+1, n]}$}

We use the notation of the previous section. The relative canonical bundle of $W$ is denoted by $K_{W}^{\mathrm{rel}}$.

Proposition 6.5 The following identity holds in $K^{\mathrm{rel}}\left(W_{\mathrm{rel}}^{[n+1, n]}\right)$ :

$$
\begin{aligned}
\psi ! \widetilde{\kappa}_{n+1}=\phi^{!} \widetilde{\kappa}_{n}+\mathcal{L}+\mathcal{L}^{\vee} \cdot \bar{\rho}^{!} K_{W}^{\mathrm{rel} \vee}-\bar{\rho}^{!}\left(\mathcal{O}_{W}^{\mathrm{rel}}-T^{\mathrm{rel}} W+K_{W}^{\mathrm{rel} \vee}\right) \\
-\mathcal{L} \cdot \bar{\sigma}^{!} \widetilde{\mathcal{O}}_{n}^{\vee}-\mathcal{L}^{\vee} \cdot \bar{\rho}^{!} K_{W}^{\mathrm{rel} \vee} \cdot \bar{\sigma}^{!} \widetilde{\mathcal{O}}_{n}
\end{aligned}
$$

Proof By Proposition 6.4, $\psi ! \widetilde{\kappa}_{n+1}=\psi ! \widetilde{p}_{*}\left(\widetilde{\mathcal{O}}_{n+1}+\widetilde{\mathcal{O}}_{n+1}^{\vee}-\widetilde{\mathcal{O}}_{n+1} \cdot \widetilde{\mathcal{O}}_{n+1}^{\vee}\right)$. Let us consider the cartesian diagrams:

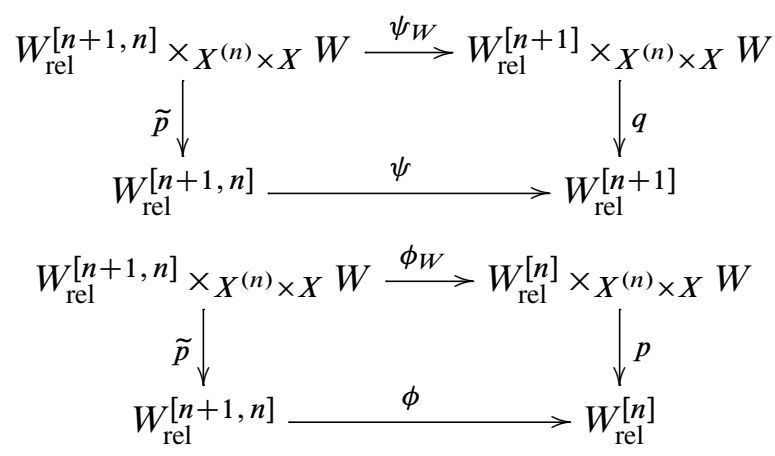

Since $p$ (resp. $q$ ) is finite on $\widetilde{\mathfrak{Y}}_{n}$ (resp. $\widetilde{\mathfrak{Y}}_{n+1}$ ), Proposition 6.4 and Proposition 3.16 (iv) yield

and

$$
\psi ! \widetilde{\kappa}_{n+1}=\tilde{p}_{*} \psi_{W}^{!}\left(\widetilde{\mathcal{O}}_{n+1}+\widetilde{\mathcal{O}}_{n+1}^{\vee}-\widetilde{\mathcal{O}}_{n+1} \cdot \tilde{\mathcal{O}}_{n+1}^{\vee}\right)
$$

$$
\phi ! \widetilde{\kappa}_{n}=\tilde{p}_{*} \phi_{W}^{!}\left(\widetilde{\mathcal{O}}_{n}+\widetilde{\mathcal{O}}_{n}^{\vee}-\widetilde{\mathcal{O}}_{n} \cdot \widetilde{\mathcal{O}}_{n}^{\vee}\right) .
$$

Thus, we obtain by Proposition 3.25 and Proposition 3.16 (ii):

$$
\begin{array}{r}
\psi ! \widetilde{\kappa}_{n+1}=\phi ! \widetilde{\kappa}_{n}+\tilde{p}_{*}\left[\tilde{p}^{!} \mathcal{L} \cdot \bar{\rho}_{W}^{!} \mathcal{O}_{\Delta}+\tilde{p}^{!} \mathcal{L}^{\vee} \cdot \bar{\rho}_{W}^{!} \mathcal{O}_{\Delta}^{\vee}-\tilde{p}^{!}\left(\mathcal{L} \cdot \mathcal{L}^{\vee}\right) \cdot \bar{\rho}_{W}^{!}\left(\mathcal{O}_{\Delta} \cdot \mathcal{O}_{\Delta}^{\vee}\right)\right. \\
\left.-\tilde{p}^{!} \mathcal{L} \cdot \bar{\rho}_{W}^{!} \mathcal{O}_{\Delta} \cdot \phi_{W}^{!} \widetilde{\mathcal{O}}_{n}^{\vee}-\tilde{p}^{!} \mathcal{L}^{\vee} \cdot \bar{\rho}_{W}^{!} \mathcal{O}_{\Delta}^{\vee} \cdot \phi_{W}^{!} \widetilde{\mathcal{O}}_{n}\right]
\end{array}
$$

Note that $\mathcal{L} \cdot \mathcal{L}^{\vee}=\mathcal{O}_{W_{\text {rel }}^{[n+1, n]}}^{\text {rel }}$. Let $\pi: W \times_{X^{(n)} \times X} W \longrightarrow W$ be the first projection. Since $\pi$ is injective on $\Delta_{\text {rel }}$, the diagram

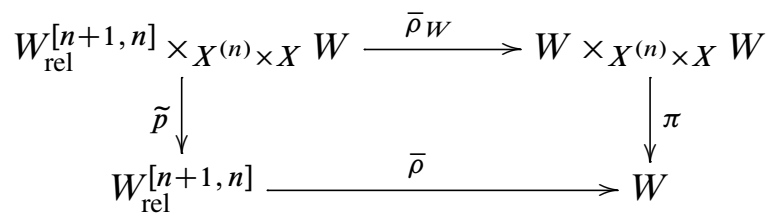


and Propositions 3.16 (iv) and 3.25 (ii) give

$$
\begin{aligned}
\psi ! & \widetilde{\kappa}_{n+1}=\phi ! \widetilde{\kappa}_{n}+\mathcal{L} \cdot \bar{\rho}^{!} \pi_{*} \mathcal{O}_{\Delta}+\mathcal{L}^{\vee} \cdot \bar{\rho}^{!} \pi_{*} \mathcal{O}_{\Delta}^{\vee}-\bar{\rho}^{!} \pi_{*}\left(\mathcal{O}_{\Delta} \cdot \mathcal{O}_{\Delta}^{\vee}\right) \\
& -\tilde{p}_{*}\left(j_{*} \mathcal{L} \cdot \phi_{W}^{!} \widetilde{\mathcal{O}}_{n}^{\vee}\right)-\mathcal{L}^{\vee} \cdot \tilde{p}_{*}\left[\bar{\rho}_{W}^{!} \mathcal{O}_{\Delta}^{\vee} \cdot \phi_{W}^{!} \widetilde{\mathcal{O}}_{n}\right] .
\end{aligned}
$$

Now $\pi_{*} \mathcal{O}_{\Delta}=\mathcal{O}_{W}^{\text {rel }}, \pi_{*} \mathcal{O}_{\Delta}^{\vee}=K_{W}^{\text {rel } \vee}$ and $\mathcal{O}_{\Delta} \cdot \mathcal{O}_{\Delta}^{\vee}=\mathcal{O}_{W}^{\text {rel }}-T_{W}^{\text {rel }}+K_{W}^{\text {rel } \vee}$, and if $\delta: W \longrightarrow W \times{ }_{X}^{(n)} \times X W$ is the diagonal injection, then $\bar{\rho}_{W}^{!} \mathcal{O}_{\Delta}^{\vee}=\bar{\rho}_{W}^{!} \delta_{*} K_{W}^{\text {rel } \vee}=$ $j_{*} \bar{\rho}^{!} K_{W}^{\mathrm{rel} \vee}$ thanks to Proposition $3.16(\mathrm{v})$ and to the diagram:

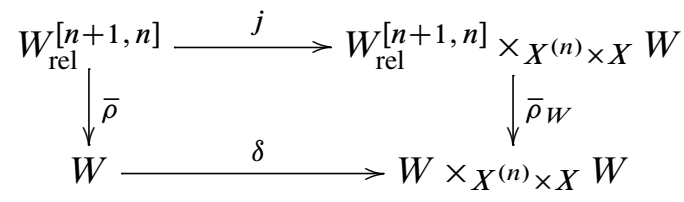

By Proposition 3.16 (ii) and (iii), we get

$$
\begin{aligned}
\psi ! & \widetilde{\kappa}_{n+1}=\phi ! \widetilde{\kappa}_{n}+\mathcal{L}+\mathcal{L}^{\vee} \cdot \bar{\rho}^{!} K_{W}^{\mathrm{rel} \vee}-\bar{\rho}^{!}\left(\mathcal{O}_{W}^{\mathrm{rel}}-T_{W}^{\mathrm{rel}}+K_{W}^{\mathrm{rel} \vee}\right) \\
& -(\tilde{p} \circ j)_{*}\left[\mathcal{L} \cdot\left(\phi_{W} \circ j\right)^{!} \widetilde{\mathcal{O}}_{n}^{\vee}\right]-\mathcal{L}^{\vee} \cdot(\tilde{p} \circ j)_{*}\left(\phi_{W} \circ j\right)^{!} \widetilde{\mathcal{O}}_{n} .
\end{aligned}
$$

Since $\tilde{p} \circ j=\mathrm{id}$ and $\phi_{W} \circ j=\bar{\sigma}$, we obtain the result.

\subsection{Cohomological computations}

Lemma 6.6 If $i$ is a positive integer, the following identities hold in $H^{2 i}\left(X^{[n+1, n]}, \mathbb{Q}\right)$ :

(i) $\left.c_{i}\left(\phi^{!} \widetilde{\kappa}_{n}\right)\right|_{X^{[n+1, n]}}=\lambda^{*} c_{i}\left(X^{[n]}\right)$ and $\left.c_{i}\left(\psi^{!} \widetilde{\kappa}_{n+1}\right)\right|_{X^{[n+1, n]}}=v^{*} c_{i}\left(X^{[n+1]}\right)$.

(ii) $\left.c_{i}\left[\left(\bar{\sigma}^{!} \widetilde{\mathcal{O}}_{n}\right)^{\infty}\right]\right|_{X^{[n+1, n]}}=\sigma^{*} \mu_{i, n}$.

(iii) $\left.c_{i}\left(\bar{\rho}^{*} T^{\mathrm{rel}} W\right)\right|_{X} ^{[n+1, n]}=\rho^{*} c_{i}(X)$.

Proof (i) By the homotopy invariance of topological K-theory, the cohomology class $\left.c_{i}\left(\phi^{!} \widetilde{\kappa}_{n}\right)\right|_{X^{[n+1, n]}}$ in $H^{2 i}\left(X^{[n+1, n]}, \mathbb{Q}\right)$ is independent of $J_{n \times 1}^{\text {rel }}$. Thus, we can assume that $W$ and $W^{\prime}$ satisfy the compatibility condition (A) of Section 2.4. It follows from Lemma 6.2 that $\left.c_{i}\left(\phi^{!} \widetilde{\kappa}_{n}\right)\right|_{X^{[n+1, n]}}=\left.\lambda^{*} c_{i}\left(\kappa_{n}\right)\right|_{X^{[n]}}=\lambda^{*} c_{i}\left(X^{[n]}\right)$. Using the compatibility condition (B) instead of (A) we obtain the second identity.

(ii) By Proposition 3.20, $\left[\left(\bar{\sigma}^{!} \widetilde{\mathcal{O}}_{n}\right)^{\infty}\right]=\bar{\sigma}^{*}\left[\widetilde{\mathcal{O}}_{n}{ }^{\infty}\right]$. This gives the result. 
(iii) Let $\bar{W}=W \times X^{(n)} X$, where the base change morphism is given by the diagonal injection of $X$ in $X^{(n)}$. We consider the diagram:

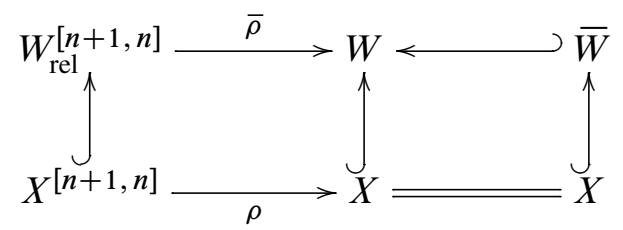

Then, $\left.c_{i}\left(\bar{\rho}^{*} T^{\mathrm{rel}} W\right)\right|_{X} ^{[n+1, n]}=\left.\rho^{*} c_{i}\left(T^{\mathrm{rel}} \bar{W}\right)\right|_{X}$. Since $\bar{W}$ is a neighbourhood of $\Delta_{X}$ in $X \times X,\left.T^{\mathrm{rel}} \frac{X}{W}\right|_{X} \simeq T X$, so that $\left.c_{i}\left(T^{\mathrm{rel}} \bar{W}\right)\right|_{X}=c_{i}(X)$.

For any nonnegative integer $k$, denote by $d_{k}$ the $k$-th Chern class of $\left[\mathcal{C}_{\Delta_{X}}^{\infty}\right]$ in $H^{2 k}(X \times X, \mathbb{Q})$. If $i: X \longrightarrow X \times X$ is the diagonal injection, the differentiable Grothendieck-Riemann-Roch theorem for immersions [3, Theorem 3.3] gives $d_{0}=1$, $d_{1}=0, d_{2}=-i_{*}(1), d_{3}=-i_{*}\left[c_{1}(X)\right]$ and $d_{k}=0$ for $k \geq 4$. Thus $i^{*} d_{0}=1$, $i^{*} d_{1}=0, i^{*} d_{2}=-c_{2}(X), i^{*} d_{3}=-c_{1}(X) c_{2}(X)$ and $i^{*} d_{k}=0$ for $k \geq 4$.

Proposition 6.7 Let $i$ be a positive integer.

(i) $(v, \text { id })^{*} \mu_{i, n+1}-(\lambda, \text { id })^{*} \mu_{i, n}=\sum_{k=0}^{i} \operatorname{pr}_{1}^{*} l^{k} .(\rho, \text { id })^{*} d_{i-k}$.

(ii) $v^{*} c_{i}\left(X^{[n+1]}\right)-\lambda^{*} c_{i}\left(X^{[n]}\right)$ is a universal polynomial in the classes $l, \rho^{*} c_{j}(X)$ and $\sigma^{*} \mu_{j, n}$.

Proof (i) Let $Z_{n \times 1 \times 1}$ be the incidence locus in $X \times\left(X^{(n)} \times X \times X\right)$ defined by

$$
Z_{n \times 1 \times 1}=\left\{(p ; \underline{x}, q, r) \text { in } X \times\left(X^{(n)} \times X \times X\right) \text { such that } p \in \underline{x} \cup q \cup r\right\}
$$

and let $J_{n \times 1 \times 1}^{\text {rel }}$ be a relative integrable complex structure in a neighbourhood $\widehat{W}$ of $Z_{n \times 1 \times 1}$. We can assume that $\left(W, J_{n \times 1}^{\text {rel }}\right)$ and $\left(\widehat{W}, J_{n \times 1 \times 1}^{\text {rel }}\right)$ satisfy as relative analytic spaces the compatibility condition $W \times_{X^{(n)} \times X}\left(X^{(n)} \times X \times X\right) \subseteq \widehat{W}$, the base change map being given by the diagonal injection of $X$ into $X \times X$.

Exactly as in Section 3.5, we can construct relative incidence sheaves $\widehat{\mathcal{O}}_{n}$ and $\widehat{\mathcal{O}}_{n+1}$ on $\widehat{W}_{\text {rel }}^{[n]}$ and $\widehat{W}_{\text {rel }}^{[n+1]}$ as well as a relative exceptional divisor $\widehat{D}_{\text {rel }}$ in $\widehat{W}_{\text {rel }}^{[n+1, n]}$. Let $\widehat{\phi}_{\widehat{W}}$, $\widehat{\psi}_{\widehat{W}}, \hat{\rho}_{\widehat{W}}, \hat{j}, \hat{p}, \hat{\mathcal{L}}, \widehat{\Delta}_{\text {rel }}$ and $\mathcal{O}_{\widehat{\Delta}}$ be the analogs of $\phi_{W}, \psi_{W}, \bar{\rho}_{W}, j, \tilde{p}, \mathcal{L}, \Delta_{\text {rel }}$ and $\mathcal{O}_{\Delta}$. In this context, Proposition 3.25 takes the following form:

- There is a natural exact sequence on $\widehat{W}_{\text {rel }}^{[n+1, n]} \times_{X^{(n)} \times X \times X} \widehat{W}$ relating $\widehat{\mathcal{O}}_{n}$ and $\widehat{\mathcal{O}}_{n+1}$, namely,

$$
0 \longrightarrow \hat{j}_{*} \widehat{\mathcal{L}} \longrightarrow \widehat{\psi}_{\widehat{W}}^{*} \widehat{\mathcal{O}}_{n+1} \longrightarrow \widehat{\phi}_{\widehat{W}}^{*} \widehat{\mathcal{O}}_{n} \longrightarrow 0 .
$$


- $\hat{j}_{*} \widehat{\mathcal{L}}=\hat{p}^{*} \widehat{\mathcal{L}} \otimes \hat{\rho}_{\widehat{W}}^{*} \mathcal{O}_{\widehat{\Delta}}$.

- $\hat{\rho}_{\widehat{W}}^{*} \mathcal{O}_{\widehat{\Delta}}=\hat{\rho}_{\widehat{W}}^{!} \mathcal{O}_{\widehat{\Delta}}, \quad \widehat{\psi}_{\widehat{W}}^{*} \widehat{\mathcal{O}}_{n+1}=\hat{\psi}_{\widehat{W}}^{!} \widehat{\mathcal{O}}_{n+1}$ and $\widehat{\phi}_{\widehat{W}}^{*} \widehat{\mathcal{O}}_{n}=\widehat{\phi}_{\widehat{W}}^{!} \widehat{\mathcal{O}}_{n}$.

Arguing as in Section 6.3 and using Proposition 3.20, we get $\left.c_{1}\left(\widehat{p}^{*} \widehat{\mathcal{L}}\right)\right|_{X^{[n+1, n] X}}=$ $\operatorname{pr}_{1}^{*} l$ and for any nonnegative integer $k$,

- $\left.c_{k}\left[\left(\hat{\rho}_{\widehat{W}}^{*} \mathcal{O}_{\widehat{\Delta}}\right)^{\infty}\right]\right|_{X^{[n+1, n]_{X X}}}=(\rho, \mathrm{id})^{*} d_{k}$,

- $\left.c_{k}\left[\left(\widehat{\psi}_{\widehat{W}}^{*} \widehat{\mathcal{O}}_{n+1}\right)^{\infty}\right]\right|_{X^{[n+1, n]} \times X}=(\nu, \mathrm{id})^{*} \mu_{k, n+1}$,

- $\left.c_{k}\left[\left(\widehat{\phi}_{\widehat{W}}^{*} \widehat{\mathcal{O}}_{n}\right)^{\infty}\right]\right|_{X^{[n+1, n]_{X} X}}=(\lambda, \mathrm{id})^{*} \mu_{k, n}$.

This yields the result.

(ii) This is a straightforward consequence of Proposition 6.5 and Lemma 6.6.

We are now going to perform in our context the induction step of [14]. For any subset $I$ of $\{0, \ldots, m\}$, we denote by $\operatorname{pr}_{I}$ the projection from $X^{[n+1]} \times X^{m}$ to the product of the factors indexed by $I$.

Proposition 6.8 If $m$ is a positive integer, let $P$ be a polynomial in the cohomology classes $\operatorname{pr}_{0}^{*} c_{i}\left(X^{[n+1]}\right), \mathrm{pr}_{0 k}^{*} \mu_{i, n+1}, \mathrm{pr}_{k l}^{*} d_{i}, \mathrm{pr}_{k}^{*} c_{i}(X)(1 \leq k, l \leq m)$ on $X^{[n+1]} \times X^{m}$. Then there exists a polynomial $\widetilde{P}$ depending only on $P$, in the analogous classes on $X^{[n]} \times X^{m+1}$, such that

$$
\int_{X^{[n+1]} \times X^{m}} P=\int_{X^{[n]} \times X^{m+1}} \tilde{P} .
$$

Proof We consider the incidence diagram:

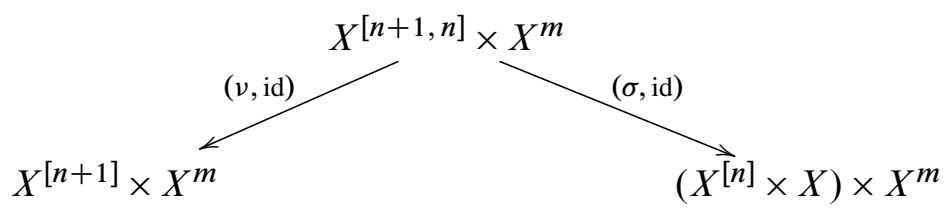

Since $(\nu$, id $)$ and $(\sigma$, id) are generically finite of degrees $n+1$ and 1 respectively,

$$
\int_{X^{[n+1]_{\times}} \times X^{m}} P=\frac{1}{n+1} \int_{X^{[n]} \times X^{m+1}}(\sigma, \mathrm{id})_{*}\left[(\nu, \mathrm{id})^{*} P\right] .
$$

Let $i$ be a positive integer. The class $(v, \mathrm{id})^{*} \operatorname{pr}_{0}^{*} c_{i}\left(X^{[n+1]}\right)-(\sigma, \mathrm{id})^{*} \operatorname{pr}_{0}^{*} c_{i}\left(X^{[n]}\right)$ is, by Proposition 6.7 (ii), a polynomial in the classes $\operatorname{pr}_{0}^{*} l,(\sigma, \mathrm{id})^{*} \operatorname{pr}_{1}^{*} c_{j}(X)$ and $(\sigma, \mathrm{id})^{*} \operatorname{pr}_{01}^{*} \mu_{j, n}$; and Proposition 3.27 gives $(\sigma, \mathrm{id})_{*} \operatorname{pr}_{0}^{*} l^{j}=(-1)^{j} \operatorname{pr}_{01}^{*} \mu_{j, n}$. Thus, 
$(\sigma, \mathrm{id})_{*}(v, \mathrm{id})^{*} \operatorname{pr}_{0}^{*} c_{i}\left(X^{[n+1]}\right)-\operatorname{pr}_{0}^{*} c_{i}\left(X^{[n]}\right)$ is a polynomial in the classes $\operatorname{pr}_{01}^{*} \mu_{j, n}$ and $\operatorname{pr}_{1}^{*} c_{j}(X)$.

By Proposition $6.7(\mathrm{i}),(v, \mathrm{id})^{*} \operatorname{pr}_{0 k}^{*} \mu_{i, n+1}-(\sigma, \mathrm{id})^{*} \operatorname{pr}_{0, k+1}^{*} \mu_{i, n}$ is a polynomial in the classes $\operatorname{pr}_{0}^{*} l$ and $(\sigma, \mathrm{id})^{*} \operatorname{pr}_{1, k+1}^{*} d_{j}$. Applying Proposition 3.27 again, we obtain that $(\sigma, \mathrm{id})_{*}(v, \mathrm{id})^{*} \operatorname{pr}_{0 k}^{*} \mu_{i, n+1}-\operatorname{pr}_{0, k+1}^{*} \mu_{i, n}$ is a polynomial in the classes $\operatorname{pr}_{01}^{*} \mu_{j, n}$ and $\operatorname{pr}_{1 k}^{*} d_{j}$.

To conclude, we use the relations

$$
\begin{aligned}
(\nu, \mathrm{id})^{*} \operatorname{pr}_{k l}^{*} d_{i} & =(\sigma, \mathrm{id})^{*} \operatorname{pr}_{k+1, l+1}^{*} d_{i}, \\
(\nu, \mathrm{id})^{*} \operatorname{pr}_{k}^{*} c_{i}(X) & =(\sigma, \mathrm{id})^{*} \operatorname{pr}_{k+1}^{*} c_{i}(X) .
\end{aligned}
$$

We can now finish the proof of Theorem 6.1. We write

$$
\int_{X^{[n]}} P\left(c_{1}\left(X^{[n]}\right), \ldots, c_{2 n}\left(X^{[n]}\right)\right)=\int_{X^{[n-1]} \times X} \tilde{P}_{1}=\int_{X^{[n-2]} \times X^{2}} \tilde{P}_{2}=\cdots=\int_{X^{n}} \tilde{P}
$$

where $\widetilde{P}$ is a universal polynomial in the classes $\operatorname{pr}_{k}^{*} c_{i}(X)$ and $\operatorname{pr}_{k l}^{*} d_{i}$. By the explicit expression of the classes $d_{i}$, we obtain that $\int_{X^{n}}^{k} \widetilde{P}$ is a universal polynomial in the Chern numbers $c_{1}(X)^{2}$ and $c_{2}(X)$.

\section{Appendix: Existence of relative integrable complex struc- tures}

This appendix is devoted to the proof of Proposition 2.13. This proof is carried out in several steps. We introduce at first some notation and terminology:

- If $Y$ and $A$ are two subsets of $X \times B$ and $B$ respectively, we put $\left.Y\right|_{A}=$ $Y \cap(X \times A)$.

- If $Y$ is a subset of $X \times B$, we say that $Y$ is adapted to $Z$ if $Y$ contains $\operatorname{pr}_{2}^{-1}\left[\operatorname{pr}_{2}(Y) \cap Z\right]$.

- If $W$ and $W^{\prime}$ are open subsets of $X \times B$, we write $W \lesssim W^{\prime}$ if $W \cap W^{\prime}$ is nonempty and if $\left.W\right|_{\operatorname{pr}_{2}(W) \cap \operatorname{pr}_{2}\left(W^{\prime}\right)}$ is included in $\left.W^{\prime}\right|_{\operatorname{pr}_{2}(W) \cap \mathrm{pr}_{2}\left(W^{\prime}\right)}$.

Then we have:

Lemma 7.1 Let $K$ be a compact subset of $B$. Then for any neighbourhood $W$ of $\left.Z\right|_{K}$ in $X \times B$, there exists a neighbourhood $U$ of $K$ in $B$ such that $\left.W\right|_{U}$ is adapted to $Z$.

Proof Let $\pi: Z \longrightarrow B$ be the restriction of the first projection to $Z$. Since $\pi$ is finite, $\pi$ is closed. Thus, if $U=B \backslash \pi[((X \times B) \backslash W) \cap Z], U$ is an open neighbourhood of $K$ in $B$, and $\left.W\right|_{U}$ is adapted to $Z$. 
We are now going to study in detail some very special open subsets of $X \times B$, which are essentially tubular neighbourhoods of $Z$. The construction of these open sets relies on the (probably well-known) lemma:

Lemma 7.2 Let $Y$ be a differentiable orbifold endowed with its natural stratification, $S$ be a stratum of $T$ and $K$ be a compact subset of $Y$. Then there exist a neighbourhood $U$ of $K$ in $Y$ and a smooth retraction $R: U \longrightarrow U \cap S$.

Proof We start by a local construction. Let $x$ be a point in $S$ and $U_{x}$ be a neighbourhood of $x$ in $Y$ such that $U_{x}$ is isomorphic as an orbifold to $V / G$, where $V$ is an open subset of $\mathbb{R}^{n}$ and $G$ is a finite group of diffeomorphisms of $V$. Let $\pi: V \longrightarrow U_{x}$ be the quotient map and $y$ be an element of $\pi^{-1}(x)$. We can assume that $G$ is equal to the stabilizer $G_{y}$ of $y$. In this case, $\pi^{-1}\left(S \cap U_{x}\right)$ is exactly the subset of the points of $V$ fixed by all the elements of $G$; we denote it by $V^{G}$.

Let us construct an embedding of $U_{x}$ into an Euclidean space. By Bochner's linearization theorem, we can assume that the finite group $G$ acts linearly on $V$. This means that the action of $G$ is induced by a linear representation of $G$ in $\operatorname{GL}(n, \mathbb{R})$ if we choose $y$ as the origin of $\mathbb{R}^{n}$. Then $G$ also acts on the algebra $\mathbb{R}\left[X_{1}, \ldots, X_{n}\right]$ of polynomial functions on $\mathbb{R}^{n}$ and the algebra $\mathbb{R}\left[X_{1}, \ldots, X_{n}\right]^{G}$ of $G$-invariant polynomials is finitely generated [40, Appendix 4, Proposition 1]. Let $\phi_{1}, \ldots, \phi_{d}$ be a set of generators and $\phi=\left(\phi_{1}, \ldots \phi_{d}\right)$. The map $\phi: \mathbb{R}^{n} \longrightarrow \mathbb{R}^{d}$ induces a smooth map $\widetilde{\phi}$ from $\mathbb{R}^{n} / G$ to $\mathbb{R}^{d}$ which is easily checked to be injective. Since $U_{x}$ is open in $\mathbb{R}^{n} / G, \widetilde{\phi}$ gives an embedding of $U_{x}$ in $\mathbb{R}^{d}$.

Let us now prove that the restriction of $\tilde{\phi}$ to $S \cap U_{x}$ is an immersion. Since $\left.\pi\right|_{V^{G}}: V^{G} \longrightarrow S \cap U_{x}$ is a diffeomorphism, this is equivalent to show that the restriction of $\phi$ to $V^{G}$ is an immersion. Let $p$ be a point in $V^{G}, h$ be a tangent vector in $T_{p} V^{G}$, and assume that $\phi_{*}(h)=0$. If we put $\psi_{i}=\phi_{i}-\phi_{i}(p)$ for $1 \leq i \leq d$, then every $G$-invariant polynomial $P$ on $\mathbb{R}^{n}$ can be decomposed as $P_{1} \psi_{1}+\cdots+P_{d} \psi_{d}+P(p)$, where the $P_{i}$ 's are $G$-invariant. This proves that $P_{*}(h)=0$. Let $A$ be the algebra of polynomial functions on the vector space $\left(\mathbb{R}^{n}\right)^{G}$. Then $A$ is a quotient of $\mathbb{R}\left[X_{1}, \ldots, X_{n}\right]$ and $G$ acts trivially on $A$, so that $A$ is a quotient of $\mathbb{R}\left[X_{1}, \ldots, X_{n}\right]^{G}$. Let us choose a linear form $u$ on $\left(\mathbb{R}^{n}\right)^{G}$ such that, via the identification between $T_{p} V^{G}$ and $\left(\mathbb{R}^{n}\right)^{G}, u(h)$ is nonzero. If $\tilde{u}$ is a lift of $u$ in $\mathbb{R}\left[X_{1}, \ldots, X_{n}\right]^{G}$, then $\tilde{u}_{*}(h)$ is nonzero, which is a contradiction.

We can now argue as in the proof of the Whitney embedding theorem in the compact case: using a partition of unity, we obtain an embedding $\Gamma$ of a neighbourhood $U$ of $K$ into some Euclidean space $\mathbb{R}^{N}$ such that $\left.\Gamma\right|_{U \cap S}$ is an immersion. Since $\Gamma(U \cap S)$ 
is a submanifold of $\mathbb{R}^{N}$, after shrinking $U$ if necessary, we can consider a tubular neighbourhood $\Omega$ of $\Gamma(U \cap S)$ in $\mathbb{R}^{N}$ such that $\Gamma(U)$ is included in $\Omega$. If $\widetilde{R}: \Omega \longrightarrow \Gamma(U \cap S)$ is the retraction associated with this tubular neighbourhood, we define $R: U \longrightarrow U \cap S$ by composing $\widetilde{R} \circ \Gamma$ with the inverse of the diffeomorphism $\left.\Gamma\right|_{U \cap S}: U \cap S \stackrel{\sim}{\longrightarrow} \Gamma(U \cap S)$.

Let $r$ be the injectivity radius of $X$. Since $X$ is compact, $r$ is positive. For any $x$ in $X$ and $\varepsilon$ satisfying $0<\varepsilon<r$, the exponential map $\exp _{g}(x)$ at $x$ associated with the Riemannian metric $g$ is a diffeomorphism between the Euclidean ball of $T_{x} X$ of radius $\varepsilon$ centered at the origin and the geodesic ball $B_{g}(x, \varepsilon)$. The former being endowed with the integrable complex structure $J_{x}$, we get a canonical integrable complex structure on the latter.

Let us fix a stratum $B_{\lambda}$ of $B$ and a compact subset $K_{\lambda}$ of $B_{\lambda}$. We denote by $d(\lambda)$ the number of sheets of the covering map $\left.\operatorname{pr}_{2}\right|_{Z_{\lambda}}: Z_{\lambda} \longrightarrow B_{\lambda}$. If $\eta$ is a sufficiently small number in ]0, $r$, there exists a small neighbourhood $V_{\lambda}$ of $K_{\lambda}$ in $B_{\lambda}$ such that for any $b$ in $V_{\lambda}$, if $\left.Z\right|_{b}=\left\{x_{1}, \ldots, x_{d(\lambda)}\right\}$, then the geodesic balls $\left(B_{g}\left(x_{i}, \eta\right)\right)_{1 \leq i \leq d(\lambda)}$ are pairwise disjoint in $X$. By Lemma 7.2, we can assume that there exist a neighbourhood $U_{\lambda}$ of $K_{\lambda}$ in $B$ such that $U_{\lambda} \cap B_{\lambda}=V_{\lambda}$ and a smooth retraction $R: U_{\lambda} \longrightarrow V_{\lambda}$. Then we put $\Omega=\bigsqcup_{b \in V_{\lambda}} \bigsqcup_{\left.x \in Z\right|_{R_{\lambda}(b)}} B_{g}(x, \eta)$. Since $\Omega$ is a neighbourhood of $\left.Z\right|_{K_{\lambda}}$, Lemma 7.1 shows that $\Omega$ is adapted to $Z$ if $U_{\lambda}$ is a sufficiently small neighbourhood of $K_{\lambda}$. We call such an open set $\Omega$ an $\eta$-neighbourhood of $Z$ above $K_{\lambda}$. It is easy to prove that if $W$ is any neighbourhood of $\left.Z\right|_{K_{\lambda}}$, then there exists an $\eta$-neighbourhood of $Z$ above $K_{\lambda}$ contained in $W$ if $\eta$ is a sufficiently small positive real number.

We now explain how to cover $Z$ in a compatible way by a finite number of $\eta-$ neighbourhoods.

Lemma 7.3 Let $W$ be an open subset of $X \times B$ adapted to $Z, B_{\lambda}$ be a stratum of $B$ such that $\partial B_{\lambda}$ is contained in $\operatorname{pr}_{2}(W)$ and $\eta_{0}$ be a positive real number. Then, for every relatively compact open subset $V$ of $\operatorname{pr}_{2}(W)$ containing $\partial B_{\lambda}$, there exist a compact subset $K_{\lambda}$ of $B_{\lambda}$, a positive real number $\eta$ smaller than $\eta_{0}$ and a $\eta$-neighbourhood $\Omega$ of $Z$ above $K_{\lambda}$ such that $\bar{B}_{\lambda} \subseteq V \cup K_{\lambda}$ and $\left.\Omega \lesssim W\right|_{V}$.

Proof Let $U$ be a relatively compact neighbourhood of $\partial B_{\lambda}$ in $\bar{B}_{\lambda} \cap V$ and put $K_{\lambda}=\bar{B}_{\lambda} \backslash U$. Then $K_{\lambda}$ is compact in $B_{\lambda}$ and $\bar{B}_{\lambda} \subseteq V \cap K_{\lambda}$. If $K^{\prime}=\left(\bar{V} \cap \bar{B}_{\lambda}\right) \backslash U$, then $K^{\prime}$ is a compact subset of $B_{\lambda}$ included in $K_{\lambda}$ and $W$ is a neighbourhood of $\left.Z\right|_{K^{\prime}}$. Thus, for $\eta$ small enough, there exists an $\eta$-neighbourhood $\Omega^{\prime}$ of $Z$ above $K^{\prime}$ contained in $W$. We can even assume that $\Omega^{\prime}=\left.\Omega\right|_{O}$, where $\Omega$ is an $\eta$-neighbourhood of $Z$ above $K_{\lambda}$ and $O$ is a small neighbourhood of $K^{\prime}$ in $B$. Since $\left.\Omega\right|_{O} \subseteq W,\left.\left.\Omega\right|_{O \cap V} \subseteq W\right|_{o \cap V}$, so that $\left.\Omega \lesssim W\right|_{V}$. 
Then we get:

Lemma 7.4 For every positive real number $\eta_{0}$, we can construct a finite covering $\left(W_{i}\right)_{1 \leq i \leq N}$ of $Z$ by open subsets of $X \times B$ such that:

- For each $i$, there exists $\eta_{i}$ in $\left.] 0, \eta_{0}\right]$ such that $W_{i}$ is a disjoint finite union of $\eta_{i}$-neighbourhoods of $Z$ above compact subsets of the strata of $B$.

- For every $i$ such that $2 \leq i \leq N, W_{i} \lesssim W_{i-1} \cup \cdots \cup W_{1}$.

- The integer $N$ is smaller than the number of strata of $B$.

Proof Let $\Lambda$ be the finite set of strata of $B$ and $\left(\Lambda_{i}\right)_{1 \leq i \leq N}$ be the partition of $\Lambda$ defined as follows: $\Lambda_{1}$ is the set of elements of $\Lambda$ corresponding to closed (ie minimal) strata in $B$, and the other $\Lambda_{i}$ 's are defined inductively for $i \geq 2$ by the expression

$$
\Lambda_{i}=\left\{\lambda \text { in } \Lambda \backslash\left(\Lambda_{1} \cup \cdots \cup \Lambda_{i-1}\right) \text { such that } \partial B_{\lambda} \subseteq \bigcup_{j=1}^{i-1} \bigcup_{\mu \in \Lambda_{j}} B_{\mu}\right\} .
$$

For $1 \leq i \leq N$, we put $S_{i}=\bigcup_{j=1}^{i} \bigcup_{\lambda \in \Lambda_{j}} B_{\lambda}$. The $S_{i}$ 's are closed subsets of $B$. Let $\Lambda_{1}=\left\{\lambda_{1}, \ldots, \lambda_{k}\right\}$. For $\eta_{1}$ sufficiently small, we can pick pairwise $\eta_{1}$-neighbourhoods of $Z$ above the $B_{\lambda_{i}^{\prime}}$ s $(1 \leq i \leq k)$. We denote their union by $W_{1}$. Then $\operatorname{pr}_{2}\left(W_{1}\right)$ is a neighbourhood of $S_{1}$ in $B$. Let $V$ be a relatively compact neighbourhood of $S_{1}$ in $\operatorname{pr}_{2}\left(W_{1}\right)$. If $\Lambda_{2}=\left\{\mu_{1}, \ldots, \mu_{l}\right\}$, we can find by Lemma 7.3 some compact subsets $K_{1}, \ldots, K_{l}$ of $B_{\mu_{1}}, \ldots, B_{\mu_{l}}$ and $\eta_{2}$-neighbourhoods $\Omega_{1}, \ldots, \Omega_{l}$ of $Z$ above $K_{1}, \ldots, K_{l}$ (where $0 \leq \eta_{2} \leq \eta_{1}$ ) such that for $1 \leq i \leq l, \bar{B}_{\mu_{i}} \subseteq V \cup K_{i}$ and $\left.\Omega_{i} \lesssim W_{1}\right|_{V}$. If $\eta_{2}$ is small enough, we can assume that the $\Omega_{i}$ 's are pairwise disjoint. If we replace $W_{1}$ by $\left.W_{1}\right|_{V}$ and if we put $W_{2}=\bigsqcup_{i=1}^{l} \Omega_{i}$, then $\operatorname{pr}_{2}\left(W_{1}\right)$ is still a neighbourhood of $S_{1}$ in $B$ and $\operatorname{pr}_{2}\left(W_{1} \cup W_{2}\right)$ is a neighbourhood of $S_{2}$ in $B$. Besides, $W_{2} \lesssim W_{1}$. To construct $W_{3}$, we add the strata in $\Lambda_{3}$ and so on.

We now turn to the construction of relative integrable complex structures. If we take a covering $\left(W_{i}\right)_{1 \leq i \leq N}$ of $Z$ given by Lemma 7.4 , each $W_{i}$ (more generally each $\eta$-neighbourhood of $Z$ above a compact subset of a stratum of $B$ ) is endowed with a canonical relative integrable complex structure, but these various structures do not match on the intersections $W_{i} \cap W_{j}$. This is why it is necessary to use a gluing argument. We start by some preliminaries.

Let $E$ be any finite-dimensional real vector space of even dimension $2 k$. If $\mathcal{J}(E)$ is the set of complex structures on $E, \mathcal{J}(E)$ is a homogeneous space isomorphic to $\operatorname{GL}(2 k, \mathbb{R}) / \operatorname{GL}(k, \mathbb{C})$, so it is a submanifold of $\operatorname{End}(E)$. We define a subset $\mathfrak{J}$ of the vector bundle $\operatorname{End}(T X)$ by $\mathfrak{J}=\bigsqcup_{x \in X} \mathcal{J}\left(T_{x} X\right)$. Then $\mathfrak{J}$ is a differentiable 
manifold and the projection $p: \mathfrak{J} \longrightarrow X$ is a smooth fibration. Besides, the almostcomplex structure $J$ of $X$ is a smooth section of this fibration. For $\varepsilon>0$ and $x$ in $X$, we put $\mathcal{J}_{\varepsilon}\left(T_{X} X\right)=\left\{M\right.$ in $\mathcal{J}\left(T_{X} X\right)$ such that $\left.\|M-J(x)\|_{g}<\varepsilon\right\}$ and we define $\mathfrak{J}_{\varepsilon}=\bigsqcup_{x \in X} \mathcal{J}_{\varepsilon}\left(T_{X} X\right)$. For any $\varepsilon>0, \mathfrak{J}_{\varepsilon}$ is a neighbourhood of the image of $J$ in $\mathfrak{J}$. Furthermore, since $X$ is compact, there exists $\varepsilon_{0}>0$ such that for every $\varepsilon$ smaller than $\varepsilon_{0}, \mathfrak{J}_{\varepsilon}$ is a smooth fibration whose fiber is diffeomorphic to an Euclidean ball.

Let $\eta$ be a positive real number strictly smaller than $r$. For $x$ in $X$ and $t$ in [0,1], we define $u_{t}: B_{g}(x, \eta) \longrightarrow B_{g}(x, t \eta)$ by $u_{t}(x)(p)=\exp _{g}(x)\left[t \exp _{g}(x)^{-1}(p)\right]$. For any $y$ in $B_{g}(x, \eta)$, there is a canonical isomorphism $\phi_{t}(x, y)$ between $T_{y} X$ and $T_{u_{t}(x)(y)} X$ obtained via the differential of $\exp _{g}(x)$ from the canonical isomorphism between $T_{\exp _{g}(x)^{-1}(y)}\left(T_{x} X\right)$ and $T_{t} \exp _{g}(x)^{-1}(y)\left(T_{x} X\right)$. Since $X$ is compact, there is a positive constant $A_{\eta}$ depending only on $\eta$ such that $\lim _{\eta \rightarrow 0} A_{\eta}=1$, and for every $x$ in $X$, every $y$ in $B_{g}(x, \eta)$ and every $t$ in $[0,1],\left\|\phi_{t}(x, y)\right\|_{g} \leq A_{\eta}$ and $\left\|\phi_{t}(x, y)^{-1}\right\|_{g} \leq A_{\eta}$. If $x$ is in $X$ and $t$ in [0,1], let $\widetilde{J}$ be an almost-complex structure on $B_{g}(x, t \eta)$; if $t=0$, we take the convention that $\widetilde{J}$ is a complex structure on $T_{x} X$. Then we can define a rescaled almost-complex structure $R_{t}(\widetilde{J})$ on $B_{g}(x, \eta)$ by the formula

$$
\forall y \in B_{g}(x, \eta), \forall h \in T_{y} X, R_{t}(\tilde{J})(y)(h)=\phi_{t}(x, y)^{-1}\left(\widetilde{J}\left(u_{t}(x)(y)\right)\left(\phi_{t}(x, y)(h)\right)\right) .
$$

Then, for every $t$ in $[0,1]$ and every almost-complex structures $\widetilde{J}_{0}$ and $\widetilde{J}_{1}$ on $B_{g}(x, t \eta)$, we have

$$
\left\|R_{t}\left(\tilde{J}_{1}\right)-R_{t}\left(\tilde{J}_{0}\right)\right\|_{C^{0}, g, B_{g}(x, \eta)} \leq A_{\eta}^{2}\left\|\tilde{J}_{1}-\tilde{J}_{0}\right\|_{C^{0}, g, B_{g}(x, t \eta)} .
$$

Remark that if $\widetilde{J}$ is an almost-complex structure on $B_{g}(x, t \eta)$, then $R_{t}(\widetilde{J})=u_{t}(x)^{*} \widetilde{J}$ if $t>0$, and $R_{t}(\tilde{J})=\exp _{g}(x)_{*}(\tilde{J}(x))$ if $t=0$. Thus, if $t>0$ and $\widetilde{J}$ is integrable or if $t=0$, then $R_{t}(\widetilde{J})$ is integrable.

We end with another estimate. For any $x$ in $X$ and any $t$ in $[0,1], R_{t}(J)(x)=$ $J(x)$. Since $X$ is compact, there exists a positive constant $B_{\eta}$ depending only on $\eta$ such that $\lim _{\eta \rightarrow 0} B_{\eta}=0$, and for every $x$ in $X$ and every $t$ in $[0,1]$, we have $\left\|J-R_{t}(J)\right\|_{C^{0}, g, B_{g}(x, \eta)} \leq B_{\eta}$.

We are ready to prove the essential gluing lemma:

Lemma 7.5 Let $\varepsilon, \varepsilon^{\prime}$ and $\eta$ be positive real numbers satisfying the conditions $\eta<r$, $0<\varepsilon^{\prime}<\varepsilon \leq \varepsilon_{0}$ and $\varepsilon^{\prime} A_{\eta}^{4}+B_{\eta}\left(A_{\eta}^{2}+1\right)<\varepsilon$, and let $W$ be a $\eta$-neighbourhood of $Z$ above a compact subset $K_{\lambda}$ of a stratum $B_{\lambda}$ of $B$. If $J_{0}^{\text {rel }}$ and $J_{1}^{\text {rel }}$ are two relative integrable complex structures in $\mathcal{B}_{g, \varepsilon^{\prime}}(W)$, then there exists a smooth family $\left(J_{s}^{\text {rel }}\right)_{0 \leq s \leq 1}$ of relative integrable complex structures in $\mathcal{B}_{g, \varepsilon}(W)$ joining $J_{0}^{\text {rel }}$ and $J_{1}^{\text {rel }}$. 
Proof Let $\widetilde{J}_{0}^{\text {rel }}=R_{0}\left(J_{0}^{\text {rel }}\right)$ and $\widetilde{J}_{1}^{\text {rel }}=R_{0}\left(J_{1}^{\text {rel }}\right)$. Then the families $\left(R_{t}\left(J_{0}^{\text {rel }}\right)\right)_{0 \leq t \leq 1}$ and $\left(R_{t}\left(J_{1}^{\mathrm{rel}}\right)\right)_{0 \leq t \leq 1}$ are two smooth families of relative integrable complex structures on $W$ joining $J_{0}^{\text {rel }}$ and $J_{1}^{\text {rel }}$ to $\widetilde{J}_{0}^{\text {rel }}$ and $\widetilde{J}_{1}^{\text {rel }}$ respectively. Besides, for every $t$ in $[0,1]$ and $i$ in $\{0,1\}$,

$$
\begin{aligned}
\left\|R_{t}\left(J_{i}^{\mathrm{rel}}\right)-J\right\|_{C^{0}, g, W} & \leq\left\|R_{t}\left(J_{i}^{\mathrm{rel}}\right)-R_{t}(J)\right\|_{C^{0}, g, W}+\left\|R_{t}(J)-J\right\|_{C^{0}, g, W} \\
& <A_{\eta}^{2} \varepsilon^{\prime}+B_{\eta} .
\end{aligned}
$$

Let $\widetilde{\varepsilon}=A_{\eta}^{2} \varepsilon^{\prime}+B_{\eta}, V=\operatorname{pr}_{2}(W) \cap B_{\lambda}$ and $R_{\lambda}: \operatorname{pr}_{2}(W) \longrightarrow V$ be the smooth retraction associated with $W$. Since $\widetilde{\varepsilon}$ is smaller than $\varepsilon_{0}, \mathfrak{J} \widetilde{\varepsilon} \times\left._{X} Z\right|_{V}$ is a smooth fibration over $\left.Z\right|_{V}$ whose fiber is diffeomorphic to an Euclidean ball (hence contractible).

The relative integrable complex structures $\widetilde{J}_{0}^{\text {rel }}$ and $\widetilde{J}_{1}^{\text {rel }}$ define two sections $J_{0}^{\prime}$ and $J_{1}^{\prime}$ of the fibration $\mathfrak{J}_{\widetilde{\varepsilon}} \times\left._{X} Z\right|_{V}$; they are given for $(x, v)$ in $\left.Z\right|_{V}$ by $J_{i}^{\prime}(x, v)=\widetilde{J}_{i, v}^{\text {rel }}(x)$. Then we can find a smooth family $\left(J_{t}^{\prime}\right)_{0 \leq t \leq 1}$ of sections of $\mathfrak{J}_{\tilde{\varepsilon}} \times\left._{X} Z\right|_{V}$ joining $J_{0}^{\prime}$ and $J_{1}^{\prime}$. Each $J_{t}^{\prime}$ defines a relative integrable complex structure $J_{t}^{\prime \text { rel }}$ on $W$ as follows: for $b$ in $\operatorname{pr}_{2}(W)$, let $v=R_{\lambda}(b)$ and $\left.Z\right|_{v}=\left\{x_{1}, \ldots, x_{d}\right\}$. Then $W_{b}=\coprod_{i=1}^{d} B_{g}\left(x_{i}, \eta\right)$ and $J_{t}^{\text {rel }}$ is equal to $R_{0}\left(J_{t}^{\prime}\left(x_{i}, v\right)\right)$ on $B_{g}\left(x_{i}, \eta\right)$. Note that $\widetilde{J}_{i}^{\text {rel }}=J_{i}^{\prime \text { rel }}$ for $i=0,1$. Besides,

$\left\|J_{t}^{\prime \text { rel }}-J\right\|_{C^{0}, g, W} \leq\left\|J_{t}^{\prime \text { rel }}-R_{0}(J)\right\|_{C^{0}, g, W}+\left\|R_{0}(J)-J\right\|_{C^{0}, g, W}<A_{\eta}^{2} \widetilde{\varepsilon}+B_{\eta}<\varepsilon$.

Thus we have found a piecewise smooth family of relative integrable complex structures in $\mathcal{B}_{g, \varepsilon}(W)$ joining $J_{0}^{\text {rel }}$ and $J_{1}^{\text {rel }}$. This family can be made smooth by reparametrization.

We can now finish the proof of Proposition 2.13. Fix $\varepsilon$ and $\varepsilon^{\prime}$ which satisfy the conditions $0<\varepsilon^{\prime}<\varepsilon \leq \varepsilon_{0}$. If $d$ is the number of strata of $B$ and $\eta$ is a positive real number, we define a sequence $\left(\varepsilon_{i}\right)_{1 \leq i \leq d}$ by $\varepsilon_{1}=\varepsilon^{\prime}$ and, for $1 \leq i \leq d-1$, $\varepsilon_{i+1}=A_{\eta}^{4} \varepsilon_{i}+B_{\eta}\left(A_{\eta}^{2}+1\right)$. Then we can find $\eta_{0}>0$ such that for every $\eta$ smaller than $\eta_{0}$, the $\varepsilon_{i}$ 's are strictly smaller than $\varepsilon$ for $1 \leq i \leq d$ and $B_{\eta}$ is strictly smaller than $\varepsilon^{\prime}$. We pick such an $\eta_{0}$ and take a covering $\left(W_{i}\right)_{1 \leq i \leq N}$ of $Z$ satisfying the conditions of Lemma 7.3. Each open subset $W_{i}$ is endowed with a relative integrable complex structure $J_{i}^{\text {rel }}$ such that $\left\|J_{i}^{\text {rel }}-J\right\|_{C^{0}, g, W_{i}}<\varepsilon^{\prime}$.

Since $W_{2} \lesssim W_{1}, W_{1} \cap W_{2}$ is a finite disjoint union of $\eta_{2}$-neighbourhoods of $Z$ (where $\eta_{2} \leq \eta_{0}$ ) above compact subsets of the strata of $B$. The relative integrable complex structures $J_{1}^{\text {rel }}$ and $J_{2}^{\text {rel }}$ being in $\mathcal{B}_{g, \varepsilon_{1}}\left(W_{1} \cap W_{2}\right)$, there exists by Lemma 7.5 a smooth family $\left(J_{t}^{\text {rel }}\right)_{1 \leq t \leq 2}$ in $\mathcal{B}_{g, \varepsilon_{2}}\left(W_{1} \cap W_{2}\right)$ joining $J_{1}^{\text {rel }}$ and $J_{2}^{\text {rel }}$.

Let $V$ be a relatively compact open subset of $\operatorname{pr}_{2}\left(W_{1}\right)$. If $V$ is large enough, the open sets $\left(\left.W_{1}\right|_{V}, W_{2}, \ldots, W_{N}\right)$ still satisfy the conditions of Lemma 7.3. Let $\chi$ be a 
smooth function from $\operatorname{pr}_{2}\left(W_{1} \cup W_{2}\right)$ to $[1,2]$ such that $\chi \equiv 1$ on $\bar{V}$ and $\operatorname{supp}(\chi-2)$ is relatively compact in $\operatorname{pr}_{2}\left(W_{1}\right)$. Then we define a relative integrable complex structure $\widetilde{J}^{\text {rel }}$ in $\mathcal{B}_{g, \varepsilon_{2}}\left(W_{1 \mid V} \cup W_{2}\right)$ by the formula $\widetilde{J}_{b}^{\text {rel }}=J_{\chi}^{\text {rel }}(b), b$ for $b$ in $V \cup \operatorname{pr}_{2}\left(W_{2}\right)$. The same argument can be used to glue $\widetilde{J}^{\text {rel }}$ and $J_{3}^{\text {rel }}$ together, and so on. Finally, we get a relative integrable complex structure in $\mathcal{B}_{g, \varepsilon_{N}}(W)$, where $W$ is a neighbourhood of $Z$ in $X \times B$. Thus $\mathcal{B}_{g, \varepsilon}$ is nonempty.

We now prove the connectedness of $\mathcal{B}_{g, \varepsilon}$ for $0<\varepsilon \leq \varepsilon_{0}$. Let $J_{0}^{\text {rel }}$ and $J_{1}^{\text {rel }}$ be two relative integrable structures in $\mathcal{B}_{g, \varepsilon}(W)$, where $W$ is a neighbourhood of the incidence set $Z$ in $X \times B$. We put $\varepsilon^{\prime}=\left\|J_{0}^{\text {rel }}-J_{1}^{\text {rel }}\right\|_{C^{0}, g, W}$. As we did previously, we can find $\eta_{0}>0$ such that for every positive $\eta$ smaller than $\eta_{0}$, the $\varepsilon_{i}$ 's are strictly smaller than $\varepsilon$ for $1 \leq i \leq d+1$. For this $\eta_{0}$, we take a covering $\left(W_{i}\right)_{1 \leq i \leq N}$ of $Z$ satisfying the conditions of Lemma 7.3 such that each $W_{i}$ is contained in $W$. Then for each $i,\left.J_{0}^{\text {rel }}\right|_{W_{i}}$ and $\left.J_{1}^{\text {rel }}\right|_{W_{i}}$ are elements of $\mathcal{B}_{g, \varepsilon_{1}}\left(W_{i}\right)$, so that, by Lemma 7.5 , there exists a smooth family $\left(J_{i, t}^{\text {rel }}\right)_{0 \leq t \leq 1}$ in $\mathcal{B}_{g, \varepsilon_{2}}\left(W_{i}\right)$ joining $\left.J_{0}^{\text {rel }}\right|_{W_{i}}$ and $\left.J_{1}^{\text {rel }}\right|_{W_{i}}$. Using exactly the same methods as before, we can glue the families $\left\{\left(J_{i, t}^{\mathrm{rel}}\right)_{0 \leq t \leq 1}\right\}_{1 \leq i \leq N}$ together and get a smooth family in $\mathcal{B}_{g, \varepsilon_{N+1}}(\widetilde{W})$ joining $J_{0}^{\text {rel }}$ to $J_{1}^{\text {rel }}$, where $\widetilde{W}$ is a neighbourhood of $Z$ in $X \times B$.

The weak contractibility follows from the connectedness of $\mathcal{B}_{g, \varepsilon}$ over the bases $B \times \mathbb{S}^{p}$.

\section{References}

[1] A Adem, J Leida, Y Ruan, Orbifolds and stringy topology, Cambridge Tracts in Math. 171, Cambridge Univ. Press (2007) MR2359514

[2] M F Atiyah, F Hirzebruch, Analytic cycles on complex manifolds, Topology 1 (1962) 25-45 MR0145560

[3] MF Atiyah, F Hirzebruch, The Riemann-Roch theorem for analytic embeddings, Topology 1 (1962) 151-166 MR0148084

[4] A Beauville, Variétés Kähleriennes dont la première classe de Chern est nulle, J. Differential Geom. 18 (1983) 755-782 (1984) MR730926

[5] A Borel, J-P Serre, Le théorème de Riemann-Roch, Bull. Soc. Math. France 86 (1958) 97-136 MR0116022

[6] J Briançon, Description de Hilb ${ }^{n}\{\{x, y\}$, Invent. Math. 41 (1977) 45-89 MR0457432

[7] M A A de Cataldo, L Migliorini, The Douady space of a complex surface, Adv. Math. 151 (2000) 283-312 MR1758249

[8] J Cheah, Cellular decompositions for nested Hilbert schemes of points, Pacific J. Math. 183 (1998) 39-90 MR1616606 
[9] W Chen, Y Ruan, A new cohomology theory of orbifold, Comm. Math. Phys. 248 (2004) 1-31 MR2104605

[10] G Danila, Sur la cohomologie d'un fibré tautologique sur le schéma de Hilbert d'une surface, J. Algebraic Geom. 10 (2001) 247-280 MR1811556

[11] S K Donaldson, Symplectic submanifolds and almost-complex geometry, J. Differential Geom. 44 (1996) 666-705 MR1438190

[12] S K Donaldson, P B Kronheimer, The geometry of four-manifolds, Oxford Math. Monogr., Oxford Science Publ., The Clarendon Press, Oxford Univ. Press, New York (1990) MR1079726

[13] D Eisenbud, Commutative algebra with a view toward algebraic geometry, Graduate Texts in Math. 150, Springer, New York (1995) MR1322960

[14] G Ellingsrud, L Göttsche, M Lehn, On the cobordism class of the Hilbert scheme of a surface, J. Algebraic Geom. 10 (2001) 81-100 MR1795551

[15] B Fantechi, L Göttsche, Orbifold cohomology for global quotients, Duke Math. J. 117 (2003) 197-227 MR1971293

[16] J Fogarty, Algebraic families on an algebraic surface, Amer. J. Math 90 (1968) 511521 MR0237496

[17] L Göttsche, The Betti numbers of the Hilbert scheme of points on a smooth projective surface, Math. Ann. 286 (1990) 193-207 MR1032930

[18] L Göttsche, Hilbert schemes of zero-dimensional subschemes of smooth varieties, Lecture Notes in Math. 1572, Springer, Berlin (1994) MR1312161

[19] L Göttsche, Hilbert schemes of points on surfaces, from: "Proceedings of the International Congress of Mathematicians, Vol. II (Beijing, 2002)", (T T Li, editor), Higher Ed. Press, Beijing (2002) 483-494 MR1957058

[20] H Grauert, R Remmert, Theory of Stein spaces, Classics in Math., Springer, Berlin (2004) MR2029201 Translated from the German by A Huckleberry, Reprint of the 1979 translation

[21] J Grivaux, Topological properties of punctual Hilbert schemes of almost-complex fourfolds, to appear in Manuscripta Math. arXiv:1001.0114

[22] J Grivaux, Quelques problèmes de géométrie complexe et presque complexe, $\mathrm{PhD}$ thesis, Université Pierre et Marie Curie (2009) Available at http:// tel. archives-ouvertes.fr/docs/00/46/03/34/PDF/These-Grivaux.pdf

[23] I Grojnowski, Instantons and affine algebras. I. The Hilbert scheme and vertex operators, Math. Res. Lett. 3 (1996) 275-291 MR1386846

[24] A A Iarrobino, Punctual Hilbert schemes, Mem. Amer. Math. Soc. 10, no. 188 (1977) MR0485867 
[25] M Lehn, Chern classes of tautological sheaves on Hilbert schemes of points on surfaces, Invent. Math. 136 (1999) 157-207 MR1681097

[26] M Lehn, C Sorger, Symmetric groups and the cup product on the cohomology of Hilbert schemes, Duke Math. J. 110 (2001) 345-357 MR1865244

[27] M Lehn, C Sorger, The cup product of Hilbert schemes for K3 surfaces, Invent. Math. 152 (2003) 305-329 MR1974889

[28] W-P Li, Z Qin, W Wang, Generators for the cohomology ring of Hilbert schemes of points on surfaces, Internat. Math. Res. Notices (2001) 1057-1074 MR1857595

[29] W-P Li, Z Qin, W Wang, Vertex algebras and the cohomology ring structure of Hilbert schemes of points on surfaces, Math. Ann. 324 (2002) 105-133 MR1931760

[30] W-P Li, Z Qin, W Wang, Stability of the cohomology rings of Hilbert schemes of points on surfaces, J. Reine Angew. Math. 554 (2003) 217-234 MR1952174

[31] B Malgrange, Division des distributions, from: "Séminaire Schwartz (1959/60), Exp. 25, Unicité du problème de Cauchy. Division des distributions", Faculté des Sciences de Paris (1960) 21-25 MR0124968

[32] B Malgrange, Ideals of differentiable functions, Tata Institute of Fund. Research Studies in Math. 3, Tata Inst. of Fund. Research, Bombay (1967) MR0212575

[33] J Milnor, On the cobordism ring $\Omega^{*}$ and a complex analogue. I, Amer. J. Math. 82 (1960) 505-521 MR0119209

[34] H Nakajima, Heisenberg algebra and Hilbert schemes of points on projective surfaces, Ann. of Math. (2) 145 (1997) 379-388 MR1441880

[35] H Nakajima, Lectures on Hilbert schemes of points on surfaces, Univ. Lecture Series 18, Amer. Math. Soc. (1999) MR1711344

[36] S P Novikov, Topological invariance of rational classes of Pontrjagin, Dokl. Akad. Nauk SSSR 163 (1965) 298-300 MR0193644

[37] Z Qin, W Wang, Hilbert schemes and symmetric products: a dictionary, from: "Orbifolds in mathematics and physics (Madison, WI, 2001)", (A Adem, J Morava, Y Ruan, editors), Contemp. Math. 310, Amer. Math. Soc. (2002) 233-257 MR1950950

[38] Y Ruan, The cohomology ring of crepant resolutions of orbifolds, from: "GromovWitten theory of spin curves and orbifolds", (T J Jarvis, T Kimura, A Vaintrob, editors), Contemp. Math. 403, Amer. Math. Soc. (2006) 117-126 MR2234886

[39] I Satake, On a generalization of the notion of manifold, Proc. Nat. Acad. Sci. USA 42 (1956) 359-363 MR0079769

[40] I R Shafarevich, Basic algebraic geometry. 1. Varieties in projective space, second edition, Springer, Berlin (1994) MR1328833 Translated from the 1988 Russian edition and with notes by M Reid 
[41] A S Tikhomirov, The variety of complete pairs of zero-dimensional subschemes of an algebraic surface, Izv. Ross. Akad. Nauk Ser. Mat. 61 (1997) 153-180 MR1609203

[42] B Uribe, Orbifold cohomology of the symmetric product, Comm. Anal. Geom. 13 (2005) 113-128 MR2154668

[43] C Voisin, On the Hilbert scheme of points of an almost complex fourfold, Ann. Inst. Fourier (Grenoble) 50 (2000) 689-722 MR1775365

[44] C Voisin, On the punctual Hilbert scheme of a symplectic fourfold, from: "Symposium in Honor of C. H. Clemens (Salt Lake City, UT, 2000)", (A Bertram, J A Carlson, H Kley, editors), Contemp. Math. 312, Amer. Math. Soc. (2002) 265-289 MR1941585

Centre de Mathématiques et Informatique, UMR CNRS 6632 (LATP), Université de Provence 39 rue Frédéric Joliot-Curie, 13453 Cedex 13 Marseille, France

jgrivaux@cmi.univ-mrs.fr

Proposed: Lothar Göttsche

Seconded: Richard Thomas, Jim Bryan

Received: 29 June 2009 Revised: 13 September 2010 